\title{
Off-diagonal deformations of Kerr metrics and black ellipsoids in heterotic supergravity
}

\author{
Sergiu I. Vacaru ${ }^{1,2,3, a}$, Klee Irwin ${ }^{1, b}$ \\ ${ }^{1}$ Quantum Gravity Research, 101 S. Topanga Canyon Blvd \# 1159, Topanga, CA 90290, USA \\ 2 Project IDEI, University "Al. I. Cuza", Iasi, Romania \\ ${ }^{3}$ Flat 4, Brefney House, Fleet Street, Ashton-under-Lyne, Lancashire OL6 7PG, UK
}

Received: 9 October 2016 / Accepted: 16 December 2016 / Published online: 9 January 2017

(C) The Author(s) 2017. This article is published with open access at Springerlink.com

\begin{abstract}
Geometric methods for constructing exact solutions of equations of motion with first order $\alpha^{\prime}$ corrections to the heterotic supergravity action implying a nontrivial Yang-Mills sector and six-dimensional, 6-d, almostKähler internal spaces are studied. In 10-d spacetimes, general parametrizations for generic off-diagonal metrics, nonlinear and linear connections, and matter sources, when the equations of motion decouple in very general forms are considered. This allows us to construct a variety of exact solutions when the coefficients of fundamental geometric/physical objects depend on all higher-dimensional spacetime coordinates via corresponding classes of generating and integration functions, generalized effective sources and integration constants. Such generalized solutions are determined by generic off-diagonal metrics and nonlinear and/or linear connections; in particular, as configurations which are warped/compactified to lower dimensions and for Levi-Civita connections. The corresponding metrics can have (non-) Killing and/or Lie algebra symmetries and/or describe $(1+2)$-d and/or $(1+3)$-d domain wall configurations, with possible warping nearly almost-Kähler manifolds, with gravitational and gauge instantons for nonlinear vacuum configurations and effective polarizations of cosmological and interaction constants encoding string gravity effects. A series of examples of exact solutions describing generic off-diagonal supergravity modifications to black hole/ellipsoid and solitonic configurations are provided and analyzed. We prove that it is possible to reproduce the Kerr and other type black solutions in general relativity (with certain types of string corrections) in the 4-d case and to generalize the solutions to non-vacuum configurations in (super-) gravity/string theories.
\end{abstract}

\footnotetext{
a e-mail: sergiu.vacaru@gmail.com

b e-mail: klee@quantumgravityresearch.org
}

\section{Contents}

1 Introduction ............... 2

2 Heterotic supergravity in nonholonomic variables . . 5

2.1 Geometric conventions on nonholonomic $2+$ $2+\ldots$ splitting . . ......... 5

2.2 The AFDM for heterotic supergravity . . . . 7

2.3 Decoupling and integration of nonholonomic equations of motion . . . . . . . . . . 8

2.3.1 Ansatz for metrics, N-connections, and gravitational polarizations . . . . . . 8

2.3.2 Ricci d-tensors and N-adapted sources . . 8

2.3.3 N-adapted sources and nonholonomically modified Einstein equations . . . . . . 9

2.3.4 The ansatz for effective matter fields in heterotic string gravity . . . . . . 9 9

2.3.5 Decoupling of nonholonomic equations of motion and effective . . . . . . 10

2.3.6 Integration of nonholonomic equations of motion by generating functions and effective sources . . . . . . . . . 11

2.3.7 A nonlinear symmetry of generating functions and effective sources . . . . . 16

2.3.8 The Levi-Civita conditions . . . . . . . 17

2.4 Small N-adapted nonholonomic stationary deformations ............... 18

3 Nonholonomic heterotic string deformations of the

Kerr metric . . . . . . . . . . . . . . 20

3.1 Preliminaries on the Kerr vacuum solution and nonholonomic variables . . . . . . . . . . 21

3.2 Off-diagonal deformations of 4-d Kerr metrics by heterotic string sources . . . . . . . . 21

3.2.1 Nonholonomically string induced torsion for Kerr metrics in the 4-d sector . . . . . 22

3.2.2 Small modifications of Kerr metrics and effective string sources . . . . . . . 23 
3.3 String induced ellipsoidal 4-d deformations of the Kerr metric . . . . . . . . . . . . . 23

3.3.1 Ellipsoidal configurations with string induced cosmological constant . . . . . . . 23

3.3.2 Ellipsoid Kerr-de Sitter configurations in $R^{2}$ and heterotic string gravity . . . . . 24

3.4 Extra-dimensional off-diagonal string modifications of the Kerr solutions . . . . . . . . . . . 26

3.4.1 6-d deformations with nontrivial cosmological constant . . . . . . . . . 26

3.4.2 10-d deformations with NS 3-form and 6d almost Kähler internal spaces . . . . . 26

3.4.3 Off-diagonal solutions in standard 10-d heterotic string coordinates . . . . . . . 27

4 Outlook and concluding remarks . . . . . . . . . 30

References . . . . . . . . . . . . . . . . . 30

\section{Introduction}

The problem of constructing exact solutions (in particular, with parametric dependence on some deformation parameters) of equations of motion in ten-dimensional, 10-d, (super-) string and gravity is of great importance. Recent approaches for solutions of generalized gravitational and matter field equations in modified gravity theories (MGTs, with bi-metric/-connection structure, possible nontrivial mass terms for graviton, locally anisotropic effects etc.) including generic off-diagonal solutions in general relativity, GR, have been elaborated upon. This involves phenomenological applications in high energy physics, various approaches to quantum gravity and attempts to explain observational data in modern accelerating cosmology. For review of such subjects, we cite, respectively [1-7], on superstrings, flux compactifications, D-branes, instantons etc; [811], on geometric methods in quantum gravity; and [1230], on MGTs and applications; see also the references therein.

The supergravity/superstring and MGT gravitational field equations are formulated as sophisticated systems of nonlinear partial differential equations, PDEs. Various types of advanced analytic and numeric methods for constructing exact and approximate solutions of such equations have been explored. For GR, a number of examples of exact and physically important solutions are summarized in monographs [31,32]. In generalized (super-) gravity theories, most of the solutions with a variety of different vacua, such as in string and brane theories, comes from corresponding choices of the internal manifold. Toroidal compactifications, special geometric cases as Calabi-Yau and, more generally but with less supersymmetry, with $S U(3)$ structure manifolds were considered. The bulk of solutions in various super/noncommutative/extra-dimension/modified grav- ity theories are described by metrics, frames, and connections with coefficients of fundamental geometric/physical objects depending on one and/or two coordinates in 4-d to 10-d spacetimes. For well-known classes of solutions, diagonalizations of metrics are possible via coordinate transforms and the linear connection structures are mostly of Levi Civita, LC, and Kähler type. Additional distortions by torsion structures are also considered. Various generalizations of well-known and physically important exact solutions for the Schwarzschild, Kerr, Friedman-Lemaître-RobertsonWalker (FLRW), wormhole spacetimes etc were constructed. These classes of diagonalizable metrics (the off-diagonal terms in the Kerr solutions are determined by rotations and respective frame/coordinate systems) are generated by a certain ansatz where equations of motion are transformed into certain systems of nonlinear second order ordinary equations (ODEs), 2-d solitonic equations etc. These systems of ODEs have Killing vector symmetries which result in additional parametric symmetries [33-35] and depend on integration constants.

A number of physically important solutions with black hole, wormhole, cosmological, monopole, and instanton configurations etc. were constructed in different (super-) string/gravity and MGTs for a diagonalizable ansatz depending generically on one spacetime coordinate (in certain cases, with dependencies on two coordinates and with Killing or other type symmetries). For the majority of such solutions, the corresponding equations of motion transform with the corresponding diagonal ansatz for metrics and frame transforms from nonlinear systems of PDE in nonlinear systems of ODEs. The integrals of such ODEs depend on certain integration constants which are defined from some boundary/asymptotic/initial and prescribed symmetry conditions. For many years, physicists and mathematicians concentrated their efforts on constructing further generalizations and applications of "diagonalizable" solutions in string and (super-) gravity theories because it was more "easy" to find analytic and numeric solutions of resulting systems of ODEs. In these approaches, the integration constants can be related to certain physical constants considering the Cauchy problem, or by using various assumptions on asymptotic/boundary/symmetry conditions.

All versions of (supersymmetric) modified Einstein equations consist of very sophisticated off-diagonal nonlinear systems of PDEs. In general form, the main properties of nonlinear/nonholonomic/parametric interactions of such (super-) gravitational and matter field systems are described by PDEs and not by approximations to ODEs. To find general classes of solutions in analytical form, understand their geometric properties and search for possible physical implications is of great importance in modern mathematics, physics, and cosmology. Imposing only a "simple" diagonalizable ansatz of higher symmetry (as it is usually considered for generating 
new classes of exact solutions in various gravity theories), we "cut" the possibility to find infinite numbers of classes of generic off-diagonal solutions determined by generating and integrations functions depending on $3,4, \ldots$ spacetime coordinates, with various commutative and noncommutative parameters etc from the very beginning. A typical example is that of solitonic wave solutions depending on three variables, which cannot be generated for a very "simple" diagonal ansatz and factorized dependent on certain generated functions. It is possible that various problems in modern acceleration cosmology (with structure formation, dark energy and dark matter, etc.) could be solved by certain (super-) string/gravity solutions related to generic off-diagonal nonlinear configurations in GR and it may not be necessary to radically modify the standard gravity and particle physics theories.

The problem of constructing generic off-diagonal exact solutions ${ }^{1}$ with coefficients of metrics and connections and other physically important geometric objects depending on three-, four- and extra-dimensional spacetime coordinates is much more difficult. For instance, there are a maximum of six independent components of a metric tensor from ten components in a 4-d (pseudo-) Riemannian spacetime. ${ }^{2}$ Any such ansatz with metrics depending on three-four spacetime coordinates transforms the Einstein equations into systems of nonlinear PDEs, which cannot be decoupled and integrated in a general analytic form if the constructions are performed with respect to local coordinate frames and for the LC-connection. The condition of zero torsion imposes various types of contractions between the coefficients of the linear connection, reference frames and various tensor fields which do not allow any general decoupling of the corresponding systems of PDEs. To generate solutions with generic off-diagonal metrics and generalized connections for higherdimensional configurations (for instance, in $5 \mathrm{~d}-10-\mathrm{d}$ string gravity and MGTs) is a technically more difficult task than in $3 \mathrm{~d}-4 \mathrm{~d}$ theories.

In a series of publications [35-44], the so-called anholonomic frame deformation method, AFDM, of constructing exact solutions in commutative and noncommutative (super-) gravity and geometric flow theories has been explored. By straightforward analytic computations, it was proven that it is possible to decouple the gravitational field equations and generate general classes of solutions in various theories of gravity with metric and nonlinear, $\mathrm{N}-$, and linear connections structures. The geometric formalism is based on spacetime fibrations determined by nonholonomic distributions with splitting of dimensions, 2 (or 3 ) $+2+2+\cdots$. In explicit

\footnotetext{
1 The metric fields corresponding to such solutions cannot be diagonalized in a finite or infinite spacetime region via coordinate transforms.

2 Four components from a maximum of ten can be fixed to be zero using coordinate transforms, which is related to the Bianchi identities.
}

form, certain classes of $\mathrm{N}$-elongated frames of reference, the considered formal extensions/embeddings of 4-d spacetimes into higher-dimensional spacetimes are introduced and necessary types of adapted linear connections are defined. These connections are called distinguished, d-connections, defined in forms which preserve the N-connection splitting. In Einstein gravity, a d-connection is considered auxiliary, which in certain canonical forms can be uniquely defined by the metric structure following the conditions of metric compatibility and some other geometric conditions (for instance, that certain zero values for "pure" horizontal and vertical components contain nonholonomically induced torsion fields). Surprisingly, such a canonical d-connection allows us to decouple the equations of motion in general forms and generate various classes of exact solutions in generalized/modified string and gravity theories. Having constructed a class of generalized solutions in explicit form (depending on generating and integration functions, generalized effective sources and integration constants), we can constrain the induced torsion fields to zero and "extract" solutions for LC-configurations and/or Einstein gravity. It should be emphasized that it is important to impose the zero-torsion conditions at the end, i.e. after we have found a class of generalized solutions. We cannot decouple and solve the corresponding systems of PDEs in general forms if we use the LC-connection from the very beginning. Here it should be noted that it is important to work with nontrivial torsion configurations in order to find exact solutions in string gravity and gauge gravity models.

In this paper, we apply methods in the geometry of nonholonomic and almost-K ähler manifolds in order to study heterotic supergravity derived in the low-energy limit of heterotic string theory [45-47]. This publication is associated with another paper [48], where an approach to heterotic string gravity is formulated in the language of nonholonomic and almost-Kähler geometry. We cite also [1, Section 4.4] for a summary of previous results and similar conventions on warped configurations and modified gravitational equations. ${ }^{3}$ The main goal of this work is to develop a geometric method for integrating in generic off-diagonal forms, and for generalized connections, the equations of motion of heterotic supergravity, up to and including terms of order $\alpha^{\prime}$. As a secondary goal, we shall construct explicit examples of exact solutions describing nonholonomic deformations of the Kerr metric. In general, it is possible to formulate conditions for effective sources and generic off-diagonal when string gravity may encode/mimic equivalent solutions in massive gravity and/or modified $f(R, T)$ gravity. For reviews and

\footnotetext{
3 Nevertheless, we shall elaborate a different system of notations with $\mathrm{N}$-connections and auxiliary d-connections which allows us to define geometric objects on higher order shells of nonholonomically splitted 10-d spacetimes.
} 
original results related to massive and other types MGTs, we cite references [20-23,49,50].

In this work, a series of exact and/or small parameter depending solutions which for small deformations mimic rotoid Kerr-de Sitter-like black holes/ellipsoids, self-consistently embedded into generic off-diagonal backgrounds of 10-dimensional spacetimes are constructed. Such backgrounds can be of solitonic/vertex/instanton type. We study string gravity modifications with respect to nonholonomic frames and via re-definition of generating and integrations functions and coefficients of sources. These modifications can be analyzed in the framework of Einstein gravity but modeled by effective polarized cosmological constants and off-diagonal terms. Using solutions for heterotic string gravity, it is possible to mimic physically important effects in modified gravity. In a series of associated papers, we studied the acceleration of the universe, certain dark energy and dark matter locally anisotropic interactions, effective renormalization of quantum gravity models $[8,24,51]$ via nonlinear generic off-diagonal interactions on effective Einstein spaces. These constructions can developed for models elaborated in the framework of string theory.

The solutions of heterotic supergravity which are constructed in this and the associated [48] work describe $(1+3)$ dimensional walls endowed with generic, off-diagonal metrics, warped to an almost-Kähler 6-d internal space in the presence of nonholonomically deformed gravitational and gauge instantons. The generalized instanton contributions are adapted to a nontrivial nonlinear connection structure determined by generic off-diagonal interactions which allows us to solve the Yang-Mills, YM, sector and the corresponding Bianchi identity at order $\alpha^{\prime}$ (related to the gravitational constant in 10-d). Such 10-d solutions preserve two real supercharges, which correspond to the $\mathcal{N}=1 / 2$ supersymmetry. The almost-Kähler internal 6-d structure can be defined for various classes of solutions in 10-d gravity if we prescribe an effective Lagrange type generating function. In this approach we can work both with real nonholonomic gravitational and YM instanton configurations to consider deformed $S U(3)$ structures.

The paper is organized as follows: We begin Sect. 2 with a summary on formulation of the heterotic supergravity theory in nonholonomic variables which was performed in [48]. This will allow us to derive a general decoupling property of motion equations in further sections. The geometric formalism on nonlinear and distinguished connections and adapted metrics to nonholonomic $2+2+2+\cdots$ splitting of higherdimension spacetimes is outlined. We develop the AFDM as a geometric method for constructing exact solutions of equations of motion in heterotic string theory and related $4 \mathrm{~d}-$ $10 \mathrm{~d}$ modified Einstein gravitational equations. The contributions of gauge-like NS 3-forms, curvature of interior almost-
Kä hler configurations, effective scalar and gauge fields etc. are encoded into certain effective sources and generic off-diagonal terms of metrics, with effective N-connection structure, defining a nontrivial vacuum structure. Such generalized/modified gravitational equations are formulated in adapted variables and with a very general off-diagonal ansatz for the metrics, (non-) linear connections and effective matter fields, which allows a decoupling of corresponding nonlinear systems of PDEs in very general forms. We show that using this nonholonomic decoupling property, it is possible to construct classes of exact solutions depending on various sets of generating and integration functions and integrations constants, on all 10-d spacetime coordinates. The existence of a very important nonlinear symmetry is proven. This allows a re-definition of the generating functions and effective sources to other equivalent data with effective cosmological constants induced by off-diagonal, or warped, and effective sources interactions. It is shown how the geometric constructions can be performed for the "simplest" case of one Killing symmetry in the 4-d case and then generalized to non-Killing configurations and higher dimensions. The conditions of generating solutions with zero torsion are analyzed. A self-consistent formalism of constructing small $\mathrm{N}$-adapted nonholonomic stationary deformations is also explored.

Section 3 is devoted to a rigorous geometric study of nonholonomic generic off-diagonal deformations of exact solutions in heterotic string gravity containing the Kerr solution as a "primary" configuration. We show by using the AFDM it is possible to generate the Kerr solution as a particular nonholonomically constrained case and small parametric deformations with a well-defined physical interpretation. Then we construct solutions with general off-diagonal deformations of the Kerr metrics in $4 d$ to $10 d$ effective gravity with heterotic string modifications. We provide examples of (non-Einstein) metrics with nonholonomically induced torsions and study small off-diagonal modifications of the Kerr metrics determined by warped and general almost-Kähler internal space structures. Separate subsections are devoted to ellipsoidal 4d, 6-d, and 10-d deformations of the Kerr metric resulting in target vacuum rotoid, or Kerr-de Sitter, configurations, all self-consistently defining exact solutions of equations of motion in heterotic string theory.

Finally (in Sect. 4), we summarize the paper, provide conclusions and speculate on physical meanings of solutions with generic off-diagonal metrics and generalized connections constructed using the AFDM for the heterotic string theory. 


\section{Heterotic supergravity in nonholonomic variables}

In this section, we outline a nonholonomic geometric approach to the heterotic supergravity modeled in the lowenergy limit of heterotic string theory as a $\mathcal{N}=1$ and 10d supergravity coupled to super Yang-Mills theory; see for details [48]. The nonholonomic variables will be parameterized in forms which allow a general decoupling of equations of motion and generating exact solutions depending, in principle, on all spacetime coordinates. Such a higher-dimension spacetime is modeled as a $10-\mathrm{d}$ manifold $\mathcal{M}$, equipped with a Lorentzian metric $\stackrel{g}{g}$ of signature $(++-+++++++)$, with a time-like third coordinate. The heterotic supergravity theory is defined by a couple $(\mathcal{M}, \check{g})$, an NS 3-form $\check{H}$, a dilaton field $\check{\phi}$ and a gauge connection ${ }^{A} \check{\nabla}$, with gauge group $S O$ (32) or $E_{8} \times E_{8}$. In our approach, we elaborate a system of notations which is adapted correspondingly for applications of geometric methods of constructing exact solutions in [40-44]. The notations in [48] and this work are different from the "standard" system of notations in string theory (see, for instance, [1]). A so-called nonholonomic "shell by shell", or $2+2+\cdots$, splitting should be elaborated with corresponding left shell labels and shell indices. This minimizes the procedure of separating the equations of motion in certain general forms and for constructing exact solutions. In "shell" diadic variables, certain important (non) linear symmetries are explicitly shown and the type of generating and integration functions which can be considered are emphasized. Using only standard 4-d, 6-d and/or 10-d indices, like in former superstring and supergravity work, it is not possible to understand how the AFDM can be applied for generating off-diagonal solutions in (super-) string/gravity theories.

\subsection{Geometric conventions on nonholonomic $2+2+\cdots$} splitting

For geometric spacetime models on a 10-d pseudo-Riemannian spacetime $\mathcal{M}$ with a time-like coordinate $u^{3}=t$ and other coordinates being space-like, ${ }^{4}$ we consider conventional splitting of dimensions $\operatorname{dim} \mathcal{M}=4+2 s=10$; $s=$ $0,1,2,3$. The AFDM, allows us to construct exact solutions with arbitrary signatures of metrics $\check{g}$, but our goal is to consider extra-dimensional space generalizations of the Einstein theory to heterotic supergravity models. In most general forms, this is possible if we use the formalism of nonlinear connection splitting for higher-dimensional (super-) spaces and strings, which was originally considered in (super-) Lagrange-Finsler theory $[53,54]$. We shall not consider Finsler type gravity in this work, but we follow an approach

\footnotetext{
4 This parameterization of coordinates is convenient for constructing various classes of stationary solutions with warping on coordinate $u^{4}$ in a "minimal" form.
}

re-defined for nonholonomic distributions on (super-) manifolds $[40,43,44]$. The same geometric technique can be applied for tangent (super-) bundles or for (super-) manifolds enabled with certain classes of nonholonomic distributions like nonholonomic frames, nonlinear connections etc. For vector/tangent (super-) bundles, certain $x$-coordinates are used on the base (super-) manifold, but $y$-coordinates are considered for the typical fiber (super-) vector space. In the case of fibrations, the $(x, y)$-coordinates are used for the definition of a fibered structure.

We consider (abstract, or coordinate) indices and coordinates $u^{\alpha_{s}}=\left(x^{i_{s}}, y^{a_{s}}\right)$ for an oriented number of twodimensional, 2-d, "shells" added to a 4-d spacetime of signature $(++-+)$. For $s=0$, we write $u^{\alpha}=\left(x^{i}, y^{a}\right)$ and then extend "shell by shell" to a local system of 10-d coordinates,

$$
\begin{aligned}
s & =0: u^{\alpha_{0}}=\left(x^{i_{0}}, y^{a_{0}}\right)=\left(x^{i}, y^{a}\right) ; s=1: u^{\alpha_{1}} \\
& =\left(x^{\alpha}=u^{\alpha}, y^{a_{1}}\right)=\left(x^{i}, y^{a}, y^{a_{1}}\right) ; \\
s & =2: u^{\alpha_{2}}=\left(x^{\alpha_{1}}=u^{\alpha_{1}}, y^{a_{2}}\right)=\left(x^{i}, y^{a}, y^{a_{1}}, y^{a_{2}}\right) \\
s & =3: u^{\alpha_{3}}=\left(x^{\alpha_{2}}=u^{\alpha_{2}}, y^{a_{3}}\right)=\left(x^{i}, y^{a}, y^{a_{1}}, y^{a_{2}}, y^{a_{3}}\right),
\end{aligned}
$$

The corresponding subsets of indices are labeled in the form: $i=i_{0}, j=j_{0}, \ldots=1,2 ; a=a_{0}, b=b_{0}, \ldots=$ 3,4 , when $u^{3}=y^{3}=t ; a_{1}, b_{1} \ldots=5,6 ; a_{2}, b_{2} \ldots=$ 7,$8 ; a_{3}, b_{3} \ldots=9,10 ;$ and, for instance, $i_{1}, j_{1}, \ldots=$ $1,2,3,4 ; i_{2}, j_{2}, \ldots=1,2,3,4,5,6 ; i_{3}, j_{3}, \ldots=1,2,3$, $4,5,6,7,8$, or we shall write only $i_{s}$. For brief denotations, we shall write ${ }^{0} u=\left({ }^{0} x,{ }^{0} y\right) ;{ }^{1} u=\left({ }^{0} u,{ }^{1} y\right)=$ $\left({ }^{0} x,{ }^{0} y,{ }^{1} y\right),{ }^{2} u=\left({ }^{1} u,{ }^{2} y\right)=\left({ }^{0} x,{ }^{0} y,{ }^{1} y,{ }^{2} y\right)$ and ${ }^{3} u=\left({ }^{2} u,{ }^{3} y\right)=\left({ }^{0} x,{ }^{0} y,{ }^{1} y,{ }^{2} y,{ }^{3} y\right)$. Here we note in modern gravity, the so-called ADM (Arnowitt-DeserMisner) formalism is largely used with $3+1$ splitting, or any $n+1$ splitting; see for details [52]. For the purposes of this work, such splittings are not convenient because it is not possible to elaborate a technique of general decoupling of the gravitational field equations and generating off-diagonal solutions. In these cases, the conventional one-dimensional "fibers" result in certain degenerate systems of equations. To construct exact solutions in four-ten-dimensional theories, it is more convenient to work with a correspondingly defined non-integrable $2+2+\cdots$ splitting; see details in $[39,40,43]$. For the heterotic supergravity, such geometric constructions are explored in greater detail in [48]. In order to connect "shell by shell" indices and coordinates to standard ones for supergravity theories (see $[1,2]$ ), we can consider small Greek indices without sub indices, and respective coordinates $x^{\mu}$, when $\alpha, \mu, \ldots=0,1, \ldots, 9$. The identification of shell coordinates with the standard ones follows such a rule: $x^{0}=u^{3}=t$ (time-like coordinate) and (for space-like coordinates): $x^{1}=u^{1}, x^{2}=u^{2}, x^{3}=u^{4}, x^{4}=$ $u^{5}, x^{5}=u^{6}, x^{6}=u^{7}, x^{7}=u^{8}, x^{8}=u^{9}, x^{9}=u^{10}$. 
On $\mathcal{M}$, we can consider local frames/bases, $e_{\alpha_{s}}=$ $\left.e^{\underline{\alpha}_{s}} \underline{\alpha}_{s}^{s} u\right) \partial / \partial u^{\underline{\alpha}_{s}}$, where the partial derivatives $\partial_{\beta_{s}}:=\partial / \partial u^{\beta_{s}}$ define local coordinate bases. We shall underline indices if it is necessary to emphasize that such values are defined with respect to a coordinate frame. The (co) frames, $e^{\alpha_{s}}=$ $e_{\underline{\alpha}_{s}}^{\alpha_{s}}\left({ }^{s} u\right) \mathrm{d} u^{\underline{\alpha}} \underline{\alpha}_{s}$, can be defined as dual to respective $e_{\alpha_{s}}$.

For our purposes, it is convenient to work with nonholonomic (non-integrable) distributions defining a $2+2+\cdots$ spacetime splitting. Such a distribution can be introduced to define a nonlinear connection, $\mathrm{N}$-connection, structure via a Whitney sum

$$
{ }^{s} \mathbf{N}: T \mathcal{M}={ }^{0} h \mathcal{M} \oplus^{0} v \mathcal{M} \oplus{ }^{1} v \mathcal{M} \oplus^{2} v \mathcal{M} \oplus^{3} v \mathcal{M} \text {. }
$$

This formula states a conventional horizontal (h) and vertical (v) "shell by shell" splitting. We shall use boldface letters in order to define spaces and geometric objects enabled/adapted

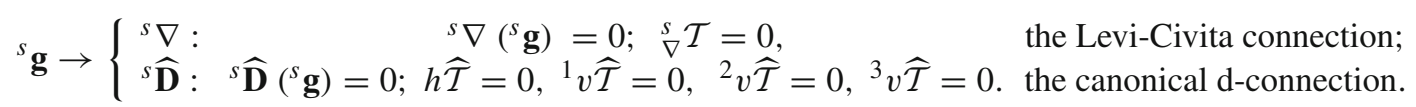

Here we note that the LC-connection ${ }^{s} \nabla=\left\{{ }_{1} \Gamma_{\beta_{s} \gamma_{s}}^{\alpha_{s}}\right\}$ can be introduced without any $\mathrm{N}$-connection structure. It can always be canonically distorted to a necessary type of d-connection ${ }^{s} \mathbf{D}$ completely defined by ${ }^{s} \mathbf{g}$ following certain geometric principles. The canonical d-connection ${ }^{s} \widehat{\mathbf{D}}$ is characterized by a nonholonomically induced torsion d-tensor which is completely defined by ${ }^{s} \mathbf{g}$ for any chosen ${ }^{s} \mathbf{N}=\left\{N_{i_{s}}^{a_{s}}\right\}$. The $\mathrm{N}$-adapted coefficients are parameterized "shell by shell" by formulas

$$
\begin{gathered}
s \widehat{\mathcal{T}}=\left\{\widehat{\mathbf{T}}_{\alpha_{s} \beta_{s}}^{\gamma_{s}}: \widehat{T}_{j_{s} k_{s}}^{i_{s}}=\widehat{L}_{j_{s} k_{s}}^{i_{s}}-\widehat{L}_{k_{s} j_{s}}^{i_{s}}, \widehat{T}_{j_{s} a_{s}}^{i_{s}}=\widehat{C}_{s_{s} b_{s}}^{i_{s}},\right. \\
\widehat{T}_{j_{s} i_{s}}^{a_{s}}=-\Omega^{a_{s}}{ }_{j_{s} i_{s}}, \widehat{T}_{a_{s} j_{s}}^{c_{s}}=\widehat{L}_{a_{s} j_{s}}^{c_{s}}-e_{a_{s}}\left(N_{j_{s}}^{c_{s}}\right), \\
\left.\widehat{T}_{b_{s} c_{s}}^{a_{s}}=\widehat{C}_{b_{s} c_{s}}^{a_{s}}-\widehat{C}_{c_{s} b_{s}}^{a_{s}}\right\} .
\end{gathered}
$$

It should be noted that this torsion is different form torsions in Einstein-Cartan gauge type and string gravity theories with absolutely antisymmetric torsion. Additional sources are not necessary because a d-torsion (7) is determined by the nonholonomic structures. In generalized theories, the torsion fields which are independent from the metric and vielbein fields may posses proper sources. Considering additional assumptions, we can relate the values, (7) for instance, to a subclass of nontrivial coefficients of an absolute antisymmetric torsion in string gravity. We can always extract LCconfigurations with zero torsion if we additionally impose for (7) the conditions

$\widehat{\mathbf{T}}_{\alpha_{s} \beta_{s}}^{\gamma_{s}}=0$, i.e. $\quad{ }^{s} \widehat{\mathbf{D}}_{\mid \widehat{T}=0} \rightarrow{ }^{s} \nabla$.

Such additional nonholonomic constraints may be stated in non-explicit forms and without certain limits with small parameters and smooth functions. It should be noted that, in 
general, $W_{\alpha_{s} \beta_{s}}^{\gamma_{s}}$ (4) may not be zero even if the conditions (8) are satisfied.

Any (pseudo-) Riemannian geometry can be equivalently formulated in nonholonomic variables $\left({ }^{s} \mathbf{g},{ }^{s} \mathbf{N},{ }^{s} \widehat{\mathbf{D}}\right)$ or using the standard data $\left({ }^{s} \mathbf{g},{ }^{s} \nabla\right)$. Because both linear connections ${ }^{s} \nabla$ and ${ }^{s} \widehat{\mathbf{D}}$ are defined by the same metric structure, there is a canonical distortion relation

${ }^{s} \widehat{\mathbf{D}}={ }^{s} \nabla+{ }^{s} \widehat{\mathbf{Z}}$.

The $\mathrm{N}$-adapted coefficients of a curvature d-tensor $\mathcal{R}_{\beta_{s}}^{\alpha_{s}}=$ $\left\{\mathbf{R}^{\alpha_{s}}{ }_{\beta_{s} \gamma_{s} \delta_{s}}\right\}$ of the canonical d-connection ${ }^{s} \widehat{\mathbf{D}}$ and ${ }^{s} \mathbf{g}$ can be computed, respectively, for all shells $s=0,1,2,3$; see for details [48]. The Ricci d-tensor $\widehat{R} i c=\left\{\widehat{\mathbf{R}}_{\alpha_{s} \beta_{s}}:=\widehat{\mathbf{R}}_{\alpha_{s} \beta_{s} \tau_{s}}^{\tau_{s}}\right\}$ is a respective contracting of the coefficients of curvature tensor,

$$
\begin{aligned}
& \widehat{\mathbf{R}}_{\alpha_{s} \beta_{s}}=\left\{\widehat{R}_{h_{s} j_{s}}:=\widehat{R}_{h_{s} j_{s} i_{s}}^{i_{s}}, \quad \widehat{R}_{j_{s} a_{s}}:=-\widehat{P}_{j_{s} i_{s} a_{s}}^{i_{s}},\right. \\
& \left.\widehat{R}_{b_{s} k_{s}}:=\widehat{P}_{b_{s} k_{s} a_{s}}^{a_{s}}, \widehat{R}_{b_{s} c_{s}}=\widehat{S}_{b_{s} c_{s} a_{s}}^{a_{s}}\right\} .
\end{aligned}
$$

Considering the inverse d-metric to ${ }^{s} \mathbf{g}$, we define and compute the scalar curvature of ${ }^{s} \widehat{\mathbf{D}}$,

$$
\begin{aligned}
{ }^{s} \widehat{R} & :=\mathbf{g}^{\alpha_{s} \beta_{s}} \widehat{\mathbf{R}}_{\alpha_{s} \beta_{s}}=g^{i_{s} j_{s}} \widehat{R}_{i_{s} j_{s}}+g^{a_{s} b_{s}} \widehat{R}_{a_{s} b_{s}} \\
& =\widehat{R}+\widehat{S}+{ }^{1} \widehat{S}+{ }^{2} \widehat{S}+{ }^{3} \widehat{S},
\end{aligned}
$$

with respective $\mathrm{h}$ - and v-components of the scalar curvature, $\widehat{R}=g^{i j} \widehat{R}_{i j}, S=g^{a b} S_{a b},{ }^{1} S=g^{a_{1} b_{1}} S_{a_{1} b_{1}},{ }^{2} S=$ $g^{a_{2} b_{2}} S_{a_{2} b_{2}},{ }^{3} S=g^{a_{3} b_{3}} R_{a_{3} b_{3}}$.

\subsection{The AFDM for heterotic supergravity}

We develop the "anholonomic frame deformation method", AFDM, and apply these geometric techniques for applications to 10-d gravity and heterotic supergravity formulated in nonholonomic variables.

The heterotic supergravity field equations were formulated in $\mathrm{N}$-adapted form in [48]. They include terms of order $\alpha^{\prime}$, equivalent to the equations of motion of heterotic nonholonomic supergravity considered in [1]. In explicit form,

$$
\begin{aligned}
& \widehat{\mathbf{R}}_{\mu_{s} v_{s}}+2\left({ }^{s} \widehat{\mathbf{D}} \widehat{\mathbf{d}} \widehat{\phi}\right)_{\mu_{s} v_{s}}-\frac{1}{4} \widehat{\mathbf{H}}_{\alpha_{s} \beta_{s} \mu_{s}} \widehat{\mathbf{H}}_{v_{s}} \alpha_{s} \beta_{s} \\
& +\frac{\alpha^{\prime}}{4}\left[\widetilde{\mathbf{R}}_{\mu_{s} \alpha_{s} \beta_{s} \gamma_{s}} \widetilde{\mathbf{R}}_{v_{s}} \alpha_{s} \beta_{s} \gamma_{s}-\operatorname{tr}\left(\widehat{\mathbf{F}}_{\mu_{s} \alpha_{s}} \widehat{\mathbf{F}}_{v_{s}} \alpha_{s}\right)\right]=0, \\
& { }^{s} \widehat{R}+4 \widehat{\square} \widehat{\phi}-4|\widehat{\mathbf{d}} \widehat{\phi}|^{2}-\frac{1}{2}|\widehat{\mathbf{H}}|^{2}+\frac{\alpha^{\prime}}{4} \operatorname{tr}\left[|\widetilde{\mathbf{R}}|^{2}-|\widehat{\mathbf{F}}|\right]=0, \\
& e^{2 \widehat{\phi}} \widehat{\mathbf{d}} \widehat{*}\left(e^{-2 \widehat{\phi}} \widehat{\mathbf{F}}\right)+\widehat{\mathbf{A}} \wedge \widehat{*} \widehat{\mathbf{F}}-\widehat{*} \widehat{\mathbf{F}} \wedge \widehat{\mathbf{A}}+\widehat{*} \widehat{\mathbf{H}} \wedge \widehat{\mathbf{F}}=0, \\
& \widehat{\mathbf{d}} \widehat{*}\left(e^{-2 \widehat{\phi}} \widehat{\mathbf{H}}\right)=0,
\end{aligned}
$$

where the Hodge operator $\widehat{*}, \widehat{ } \widehat{\mathbf{D}}=\left\{\widehat{\mathbf{D}}_{\mu_{s}}\right\}$, the canonical nonholonomic d'Alembert wave operator $\widehat{\square}:=\widehat{\mathbf{g}}^{\mu_{s} v_{s}} \widehat{\mathbf{D}}_{\mu_{s}} \widehat{\mathbf{D}}_{v_{s}}$, the Ricci d-tensor $\widehat{\mathbf{R}}_{\mu_{s} v_{s}}$, and scalar ${ }^{s} \widehat{R}$ are determined by a d-metric $\widehat{\mathbf{g}}$ (5). The curvature d-tensor $\widetilde{\mathbf{R}}_{\mu_{s} \alpha_{s} \beta_{s} \gamma_{s}}$ is taken for an almost-Kähler structure on shells $s=1,2,3$ as we described above. The gauge field $\widehat{\mathbf{A}}$ corresponds to the Nadapted operator ${ }_{A}^{S} \widehat{\mathbf{D}}$ and curvature $\widehat{\mathbf{F}}=\mathcal{F}\left({ }^{1} \psi\right)$ via a map constructed in $[1,48])$.

Equation (12) can be written as effective Einstein equations for the canonical d-connection ${ }^{s} \widehat{\mathbf{D}}$,

$$
\begin{aligned}
& { }^{s} \widehat{\mathbf{R}}_{\beta_{s} \delta_{s}}=\Upsilon_{\beta_{s} \delta_{s}}, \\
& \widehat{L}_{a_{s} j_{s}}^{c_{s}}=e_{a_{s}}\left(N_{j_{s}}^{c_{s}}\right), \quad \widehat{C}_{j_{s} b_{s}}^{i_{s}}=0, \quad \Omega_{j_{s} i_{s}}^{a_{s}}=0 .
\end{aligned}
$$

The sources $\Upsilon_{\beta_{s} \delta_{s}}$ can be formally defined as in GR but for extra dimensions and in $\mathrm{N}$-adapted form, when

$\Upsilon_{\beta_{s} \delta_{s}} \rightarrow \varkappa\left(T_{\beta_{s} \delta_{s}} \frac{1}{2} \mathbf{g}_{\beta_{s} \delta_{s}}{ }^{s} T\right)$ for ${ }^{s} \widehat{\mathbf{D}} \rightarrow{ }^{s} \nabla$.

The system (16) can be derived and formulated in variational $\mathrm{N}$-adapted form by considering a nonholonomic gravitational Lagrange density of type ${ }^{g} \widehat{L}=\widehat{\mathbf{R}}$ and an effective Lagrange density for matter ${ }^{m} \widehat{L}$. For simplicity, we shall consider Lagrangians depending only on the coefficients of metric field and matter field but not on their derivatives (for such configurations, it will be possible to construct exact solutions in explicit form). The energy-momentum d-tensor is computed by the definition,

$$
{ }^{m} \widehat{\mathbf{T}}_{\alpha \beta}:=-\frac{2}{\sqrt{\left.\right|^{s} \mathbf{g} \mid}} \frac{\delta\left(\sqrt{\left|{ }^{s} \mathbf{g}\right|}{ }^{m} \widehat{L}\right)}{\delta^{s} \mathbf{g}^{\alpha \beta}}={ }^{m} \widehat{L}^{s} \mathbf{g}^{\alpha \beta}+2 \frac{\delta\left({ }^{m} \widehat{L}\right)}{\delta^{s} \mathbf{g}_{\alpha \beta}},
$$

for $\left|{ }^{s} \mathbf{g}\right|=\operatorname{det}\left|{ }^{s} \mathbf{g}_{\mu \nu}\right|$. We conclude that by following an $\mathrm{N}$-adapted variational calculus with action

$$
{ }^{g} \mathcal{S}+{ }^{m} \mathcal{S}=\int \mathrm{d}^{4} u \sqrt{\left|{ }^{s} \mathbf{g}\right|}\left({ }^{g} \widehat{L}+{ }^{m} \widehat{L}\right),
$$

we can elaborate a 10-d nonholonomic gravity theory with gravitational field equations (16). A changing of geometric data $\left({ }^{s} \mathbf{g},{ }^{s} \widehat{\mathbf{D}}\right) \rightarrow\left({ }^{s} \mathbf{g},{ }^{\mathbf{s}} \nabla\right)$ is possible via the canonical distorting relations (9), or imposing the zero torsion condition at the end $\widehat{\mathcal{T}}=0$ (8) for extracting LC-configurations ${ }^{s} \widehat{\mathbf{D}}_{\mid \widehat{T}=0}={ }^{s} \nabla$ stated by (17). The matter Lagrange density ${ }^{m} \widehat{L}$ can be chosen in such a form that, via corresponding frame transforms, the source $\Upsilon_{\beta_{s} \delta_{s}}$ will encode the terms with the effective matter fields $\widehat{\phi}, \widehat{\mathbf{H}}_{\alpha_{s}} \beta_{s} \mu_{s}$, interior space fields $\widetilde{\mathbf{R}}_{\mu_{s} \alpha_{s} \beta_{s} \gamma_{s}}$, and gauge fields $\widehat{\mathbf{F}}_{\mu_{s} \alpha_{s}}$.

The nonholonomic equations of motion in heterotic string gravity (12) can be written in the form (16) with an effective source (related via nonlinear transforms of generating functions to certain effective cosmological constants),

$$
\begin{aligned}
& \Upsilon_{\mu_{s} v_{s}}={ }^{\phi} \Upsilon_{\mu_{s} \nu_{s}}+{ }^{H} \Upsilon_{\mu_{s} v_{s}}+{ }^{F} \Upsilon_{\mu_{s} \nu_{s}} \\
& \quad+{ }^{R} \Upsilon_{\mu_{s} v_{s}} \text {, where } \\
& { }^{\phi} \Upsilon_{\mu_{s} v_{s}}=-2\left({ }^{s} \widehat{\mathbf{D} \widehat{d} \widehat{\phi})} \mu_{\mu_{s} v_{s}} \text { with effective constant }{ }^{\phi} \Lambda ;\right. \\
& { }^{H} \Upsilon_{\mu_{s} v_{s}}=\frac{1}{4} \widehat{\mathbf{H}}_{\alpha_{s} \beta_{s} \mu_{s}} \widehat{\mathbf{H}}_{v_{s}}{ }^{{ }^{s} \beta_{s}} \text { with effective constant }{ }^{H} \Lambda ;
\end{aligned}
$$


$F \Upsilon_{\mu_{s} v_{s}}=\frac{\alpha^{\prime}}{4} \operatorname{tr}\left(\widehat{\mathbf{F}}_{\mu_{s} \alpha_{s}} \widehat{\mathbf{F}}_{v_{s}}^{\alpha_{s}}\right)$ with effective constant ${ }^{F} \Lambda$

${ }^{R} \Upsilon_{\mu_{s} v_{s}}=-\frac{\alpha^{\prime}}{4} \widetilde{\mathbf{R}}_{\mu_{s} \alpha_{s} \beta_{s} \gamma_{s}} \widetilde{\mathbf{R}}_{v_{s}}{ }^{\alpha_{s} \beta_{s} \gamma_{s}}$ with effective constant ${ }^{\widetilde{R}} \Lambda$.

The traces of the above sources, and the respective effective cosmological constants, are related via the condition (13).

2.3 Decoupling and integration of nonholonomic equations of motion

We show how the heterotic string gravitational field equations (12) written in the form (16) with sources (19) and possible constraints (17), can be formally integrated in very general forms for generic off-diagonal metrics with coefficients depending on all spacetime coordinates. Additional conditions for extracting LC-configurations with ${ }^{s} \nabla$ will be analyzed at the end after certain classes of general solutions have been constructed.

\subsubsection{Ansatz for metrics, N-connections, and gravitational polarizations}

In the simplest form, the decoupling property for any shell $s=0,1,2,3$ can be proven for certain ansatz with at least one Killing symmetry on the corresponding shell. Using an additional conformal transform on the necessary shells, we can extend the constructions for non-Killing configurations. Such a procedure is described for arbitrary finite shell $s$ in Refs. [40-43]. In this work, we state the formulas for 10-d gravity and heterotic string theory in explicit form when the prime solutions are stationary ones, i.e., do not depend on the time-like coordinate $y^{3}=t$.

Let us consider metrics of type (5), which via frame transforms $\mathbf{g}_{\alpha_{s} \beta_{s}}=e_{\alpha_{s}}^{\alpha_{s}^{\prime}} e_{\beta_{s}}^{\beta_{s}^{\prime}} \mathbf{g}_{\alpha_{s}^{\prime} \beta_{s}^{\prime}}$ can be parametrized ${ }^{5}$

$$
\begin{aligned}
{ }_{K}^{s} \mathbf{g}= & g_{i}\left(x^{k}\right) \mathrm{d} x^{i} \otimes \mathrm{d} x^{i}+h_{a}\left(x^{k}, y^{4}\right) \mathbf{e}^{a} \otimes \mathbf{e}^{b} \\
& +h_{a_{1}}\left(u^{\alpha}, y^{6}\right) \mathbf{e}^{a_{1}} \otimes \mathbf{e}^{a_{1}}+h_{a_{2}}\left(u^{\alpha_{1}}, y^{8}\right) \mathbf{e}^{a_{2}} \\
& \otimes \mathbf{e}^{b_{2}}+h_{a_{3}}\left(u^{\alpha_{2}}, y^{10}\right) \mathbf{e}^{a_{3}} \otimes \mathbf{e}^{a_{3}},
\end{aligned}
$$

where $\mathbf{e}^{a}=\mathrm{d} y^{a}+N_{i}^{a} \mathrm{~d} x^{i}$,

$$
\begin{gathered}
\text { for } N_{i}^{3}=n_{i}\left(x^{k}, y^{4}\right), N_{i}^{4}=w_{i}\left(x^{k}, y^{4}\right) ; \\
\mathbf{e}^{a_{1}}=\mathrm{d} y^{a_{1}}+N_{\alpha}^{a_{1}} \mathrm{~d} u^{\alpha}, \\
\quad \text { for } N_{\alpha}^{5}={ }^{1} n_{\alpha}\left(u^{\beta}, y^{6}\right), N_{\alpha}^{6}={ }^{1} w_{\alpha}\left(u^{\beta}, y^{6}\right) ; \\
\mathbf{e}^{a_{2}}=\mathrm{d} y^{a_{2}}+N_{\alpha_{1}}^{a_{2}} \mathrm{~d} u^{\alpha_{1}}, \\
\quad \text { for } N_{\alpha_{1}}^{7}={ }^{2} n_{\alpha_{1}}\left(u^{\beta_{1}}, y^{8}\right), N_{\alpha_{1}}^{8}={ }^{2} w_{\alpha_{1}}\left(u^{\beta_{1}}, y^{8}\right) ; \\
\mathbf{e}^{a_{3}}=\mathrm{d} y^{a_{3}}+N_{\alpha_{2}}^{a_{3}} \mathrm{~d} u^{\alpha_{2}}, \\
\quad \text { for } N_{\alpha_{2}}^{9}={ }^{3} n_{\alpha_{2}}\left(u^{\beta_{2}}, y^{10}\right), N_{\alpha_{2}}^{10}={ }^{3} w_{\alpha_{3}}\left(u^{\beta_{2}}, y^{10}\right) .
\end{gathered}
$$

\footnotetext{
$\overline{5}$ We shall put a left label $\mathrm{K}$ in order to emphasize that this is a d-metric with Killing symmetry.
}

Such an ansatz has a Killing vector $\partial / \partial y^{9}$ because the coordinate $y^{9}$ is not contained in the coefficients of such metrics. We propose that via coordinate transforms we can eliminate dependence on $y^{3}=t$ and can parametrize $h_{4}=1$ and $h_{a_{s}}={ }_{,} h\left(y^{4}\right) \breve{h}_{a_{s}}\left(x^{i}, y^{b_{s}}\right)$ for shells $s=1,2,3$, as it was considered in [48], if the configurations are with warping on $y^{4}$. The ansatz (24) can be considered as a target d-metric of a prime d-metric with flat domain wall considered in that associated paper. In this work, we restrict our considerations to stationary solutions in heterotic string gravity (which do not depend on $t$ ). Inhomogeneous and locally anisotropic cosmological configurations in such string models, with generic dependence on $t$ (see examples in [26-30]) will be studied in our future publications.

\subsubsection{Ricci d-tensors and N-adapted sources}

We suppose that via frame transforms it is always possible to introduce frame and coordinate parametrizations for ansatz (24) with ${ }^{6} \partial_{4} h_{3}=h_{3}^{*} \neq 0, \partial_{6} h_{5}=h_{5}^{* 1} \neq$ $0, \partial_{8} h_{7}=h_{7}^{* 2} \neq 0, \partial_{10} h_{9}=h_{9}^{* 3} \neq 0$. In brief, the partial derivatives are denoted, for instance, $\partial_{1} h=\partial h / \partial x^{1}=h$, $\partial_{2} h=\partial h / \partial x^{2}=h^{\prime}, \partial_{3} h=0$, and $\partial_{44} h=\partial^{2} h / \partial y^{4} \partial y^{4}=$ $h^{* *}, \partial_{66} h=\partial^{2} h / \partial y^{6} \partial y^{6}=h^{* *_{1} 1}$, etc. We shall write explicitly $\partial_{5} h=\partial h / \partial y^{5}, \partial_{6} h=\partial h / \partial x^{6}, \ldots$ without introducing "dot" and "prime" symbols for partial derivatives on shells $s=1,2,3$ but working with "star" partial derivatives on these shells, considering, respectively, $*_{1}, *_{2}, *_{3}$ if necessary written as $*_{s}$. A tedious computation of the coefficients of the canonical d-connection $\widehat{\mathbf{D}}=\left\{\widehat{\boldsymbol{\Gamma}}_{\alpha_{s} \beta_{s}}^{\gamma_{s}}\right\}$ for the ansatz (24) and then of the corresponding nontrivial coefficients of the Ricci d-tensor $\hat{\mathbf{R}}_{\alpha_{s} \beta_{s}}$ (10), see similar details in [35,3843], results in nontrivial $\mathrm{N}$-adapted coefficients:

$$
\begin{aligned}
\widehat{R}_{1}^{1}= & \widehat{R}_{2}^{2}=-\frac{1}{2 g_{1} g_{2}}\left[g_{2}-\frac{g_{1} g_{2}}{2 g_{1}}-\frac{\left(g_{2}\right)^{2}}{2 g_{2}}\right. \\
& \left.+g_{1}^{\prime \prime}-\frac{g_{1}^{\prime} g_{2}^{\prime}}{2 g_{2}}-\frac{\left(g_{1}^{\prime}\right)^{2}}{2 g_{1}}\right], \\
\widehat{R}_{3}^{3}= & \widehat{R}_{4}^{4}=-\frac{1}{2 h_{3} h_{4}}\left[h_{3}^{* *}-\frac{\left(h_{3}^{*}\right)^{2}}{2 h_{3}}-\frac{h_{3}^{*} h_{4}^{*}}{2 h_{4}}\right], \\
\widehat{R}_{3 k}= & \frac{h_{3}}{2 h_{4}} n_{k}^{* *}+\left(\frac{h_{3}}{h_{4}} h_{4}^{*}-\frac{3}{2} h_{3}^{*}\right) \frac{n_{k}^{*}}{2 h_{4}}, \\
\widehat{R}_{4 k}= & \frac{w_{k}}{2 h_{3}}\left[h_{3}^{*}-\frac{\left(h_{3}^{*}\right)^{2}}{2 h_{3}}-\frac{\left(h_{3}^{*}\right)\left(h_{4}^{*}\right)}{2 h_{4}}\right] \\
& +\frac{h_{3}^{*}}{4 h_{3}}\left(\frac{\partial_{k} h_{3}}{h_{3}}+\frac{\partial_{k} h_{4}}{h_{4}}\right)-\frac{\partial_{k}\left(h_{3}^{*}\right)}{2 h_{3}} ;
\end{aligned}
$$

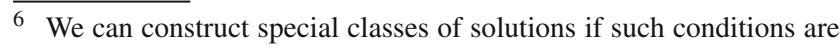
not satisfied.
} 
on shell $s=1$ with $\tau=1,2,3,4$,

$$
\begin{aligned}
\widehat{R}_{5}^{5}= & \widehat{R}_{6}^{6}=-\frac{1}{2 h_{5} h_{6}}\left[h_{5}^{*_{1} *_{1}}-\frac{\left(h_{5}^{* 1}\right)^{2}}{2 h_{5}}-\frac{\left.h_{5}^{*_{1}} h_{6}^{*_{1}}\right)}{2 h_{6}}\right], \\
\widehat{R}_{5 \tau}= & \frac{h_{5}}{2 h_{6}}{ }^{1} n_{\tau}^{*_{1}}+\left(\frac{h_{5}}{h_{6}} h_{6}^{* 1}-\frac{3}{2} h_{5}^{*_{1}}\right) \frac{{ }^{1} n_{\tau}^{* 1}}{2 h_{6}}, \\
\widehat{R}_{6 \tau}= & \frac{{ }^{1} w_{\tau}}{2 h_{5}}\left[h_{5}^{* *_{1}}-\frac{\left(h_{5}^{* 1}\right)^{2}}{2 h_{5}}-\frac{h_{5}^{* 1} h_{6}^{* 1}}{2 h_{6}}\right] \\
& +\frac{h_{5}^{* 1}}{4 h_{5}}\left(\frac{\partial_{\tau} h_{5}}{h_{5}}+\frac{\partial_{\tau} h_{6}}{h_{6}}\right)-\frac{\partial_{\tau}\left(h_{5}^{* 1}\right)}{2 h_{5}}
\end{aligned}
$$

on shell $s=2$ with $\tau_{1}=1,2,3,4,5,6$ :

$$
\begin{aligned}
\widehat{R}_{7}^{7}= & \widehat{R}_{8}^{8}=-\frac{1}{2 h_{7} h_{8}}\left[h_{7}^{*_{2} *_{2}}-\frac{\left(h_{7}^{* 2}\right)^{2}}{2 h_{7}}-\frac{h_{7}^{* 2} h_{8}^{*_{2}}}{2 h_{8}}\right], \\
\widehat{R}_{7 \tau_{1}}= & \frac{h_{7}}{2 h_{8}}{ }^{2} n_{\tau_{1}}^{*_{2} *_{2}}+\left(\frac{h_{7}}{h_{8}} h_{8}^{* 2}-\frac{3}{2} h_{7}^{* 2}\right) \frac{{ }^{2} n_{\tau_{1}}^{* 2}}{2 h_{7}}, \\
\widehat{R}_{8 \tau_{1}}= & \frac{{ }^{2} w_{\tau_{1}}}{2 h_{7}}\left[h_{7}^{*_{2} *_{2}}-\frac{\left(h_{7}^{* 2}\right)^{2}}{2 h_{7}}-\frac{h_{7}^{* 2} h_{8}^{* 2}}{2 h_{8}}\right] \\
& +\frac{h_{7}^{* 2}}{4 h_{7}}\left(\frac{\partial_{\tau_{1}} h_{7}}{h_{7}}+\frac{\partial_{\tau_{1}} h_{8}}{h_{8}}\right)-\frac{\partial_{\tau_{1}}\left(h_{7}^{* 2}\right)}{2 h_{7}},
\end{aligned}
$$

on shell $s=3$ with $\tau_{2}=1,2,3,4,5,6,7,8$ :

$$
\begin{aligned}
\widehat{R}_{9}^{9}= & \widehat{R}_{10}^{10}=-\frac{1}{2 h_{9} h_{10}}\left[h_{9}^{*^{*} *_{3}}-\frac{\left(h_{9}^{* 3}\right)^{2}}{2 h_{9}}-\frac{\left.h_{9}^{* 3} h_{10}^{* 3}\right)}{2 h_{10}}\right], \\
\widehat{R}_{9 \tau_{2}}= & \frac{h_{9}}{2 h_{10}}{ }^{2} n_{\tau_{2}}^{* 3 *_{3}}+\left(\frac{h_{9}}{h_{10}} h_{10}^{* 3}-\frac{3}{2} h_{9}^{* 3}\right) \frac{{ }^{2} n_{\tau_{2}}^{* 3}}{2 h_{9}}, \\
\widehat{R}_{10 \tau_{2}}= & \frac{{ }^{2} w_{\tau_{1}}}{2 h_{9}}\left[h_{9}^{* 3 *_{3}}-\frac{\left(h_{9}^{* 3}\right)^{2}}{2 h_{9}}-\frac{h_{9}^{* 3} h_{10}^{* 3}}{2 h_{10}}\right] \\
& +\frac{h_{9}^{* 3}}{4 h_{9}}\left(\frac{\partial_{\tau_{2}} h_{9}}{h_{9}}+\frac{\partial_{\tau_{2}} h_{10}}{h_{10}}\right)-\frac{\partial_{\tau_{2}}\left(h_{9}^{* 3}\right)}{2 h_{9}} .
\end{aligned}
$$

Using the above formulas, we can compute the Ricci scalar (11) for ${ }^{s} \widehat{\mathbf{D}}$ for the ansatz (24) and $s=0,1,2,3$ :

$$
\begin{aligned}
{ }^{0} \widehat{R} & =2\left(\widehat{R}_{1}^{1}+\widehat{R}_{3}^{3}\right),{ }^{1} \widehat{R}=2\left(\widehat{R}_{1}^{1}+\widehat{R}_{3}^{3}+\widehat{R}_{5}^{5}\right), \\
{ }^{2} \widehat{R} & =2\left(\widehat{R}_{1}^{1}+\widehat{R}_{3}^{3}+\widehat{R}_{5}^{5}+\widehat{R}_{7}^{7}\right), \\
{ }^{3} \widehat{R} & =2\left(\widehat{R}_{1}^{1}+\widehat{R}_{3}^{3}+\widehat{R}_{5}^{5}+\widehat{R}_{7}^{7}+\widehat{R}_{9}^{9}\right) .
\end{aligned}
$$

This imposes certain $\mathrm{N}$-adapted symmetries on the Einstein d-tensor for the ansatz (24); see for details [40,43].

\subsubsection{N-adapted sources and nonholonomically modified Einstein equations}

We will be able to integrate nonholonomic equations of motion in heterotic string gravity in explicit form for very general assumptions if the source $\Upsilon_{\beta_{s} \delta_{s}}$ (19) is parametrized in $\mathrm{N}$-adapted form. This can be determined by five independent effective sources, respectively, on $h$ - and ${ }^{s} v$-subspaces, which will be related to certain effective cosmological constants corresponding to the formulas

$$
\begin{aligned}
\widehat{\Upsilon}_{1}^{1} & =\widehat{\Upsilon}_{2}^{2}={ }_{h} \Upsilon\left(x^{k}\right) \rightarrow{ }_{h} \Lambda={ }_{h}^{\phi} \Lambda+{ }_{h}^{H} \Lambda+{ }_{h}^{F} \Lambda+{ }_{h}^{R} \Lambda, \\
\widehat{\Upsilon}_{3}^{3} & =\widehat{\Upsilon}_{4}^{4}=\Upsilon\left(x^{k}, y^{4}\right) \rightarrow \Lambda={ }^{\phi} \Lambda+{ }^{H} \Lambda+{ }^{F} \Lambda+{ }^{R} \Lambda, \\
\widehat{\Upsilon}_{5}^{5} & =\widehat{\Upsilon}_{6}^{6}={ }_{1} \Upsilon\left(x^{k}, y^{a}, y^{6}\right) \rightarrow{ }_{1} \Lambda \\
& ={ }_{1}^{\phi} \Lambda+{ }_{1}^{H} \Lambda+{ }_{1}^{F} \Lambda+{ }_{1}^{R} \Lambda, \\
\widehat{\Upsilon}_{7}^{7} & =\widehat{\Upsilon}_{8}^{8}={ }_{2} \Upsilon\left(x^{k}, y^{a}, y^{a_{1}}, y^{8}\right) \rightarrow{ }_{2} \Lambda \\
& ={ }_{2}^{\phi} \Lambda+{ }_{2}^{H} \Lambda+{ }_{2}^{F} \Lambda+{ }_{2}^{R} \Lambda, \\
\widehat{\Upsilon}_{9}^{9} & =\widehat{\Upsilon}_{10}^{10}={ }_{3} \Upsilon\left(x^{k}, y^{a}, y^{a_{1}}, y^{a_{2}}, y^{10}\right) \rightarrow{ }_{3} \Lambda \\
& ={ }_{3}^{\phi} \Lambda+{ }_{3}^{H} \Lambda+{ }_{3}^{F} \Lambda+{ }_{3}^{R} \Lambda .
\end{aligned}
$$

For certain general configurations, it will be possible to fix generating and effective sources of type ${ }_{h} \Lambda={ }_{s} \Lambda=\Lambda$ for all $s$, when a value for the corresponding contribution of fields can be zero, or nonzero, for instance, ${ }_{h}^{\phi} \Lambda=0$ but ${ }_{2}^{H} \Lambda \neq 0$. This depends on the type of vacuum, nonvacuum, or effective vacuum model we study. It is possible to compensate for contributions into an effective source of a field with contributions of another field, for instance, to get $\Lambda={ }^{\phi} \Lambda+{ }^{H} \Lambda+{ }^{F} \Lambda+{ }^{R} \Lambda=0$ even when not all the values of such effective cosmological constants are zero. We note that by prescribing certain values of sources (34) we can relate via nonholonomic frame transforms (in coordinate and/or N-adapted form) $\Upsilon_{\alpha_{s} \beta_{s}}=e_{\alpha_{s}}^{\alpha_{s}^{\prime}} e_{\beta_{s}}^{\beta_{s}^{\prime}} \widehat{\Upsilon}_{\alpha_{s}^{\prime} \beta_{s}^{\prime}}$, where $\Upsilon_{\alpha_{s} \beta_{s}}$ is any (effective) source (19), in heterotic string gravity, or (18), in a 10-d nonholonomic generalization of GR. For any effective ${ }^{m} \widehat{L}$ we can solve a system of quadratic algebraic equations for $e_{\alpha_{s}}^{\alpha_{s}^{\prime}}$ in a form compatible with transforms to $\mathrm{N}$-adapted frames of the metric/d-metric components,

In this section, we shall construct general solutions of the generalized nonholonomic Einstein equations (16) with Ricci d-tensors (25)-(33) and effective sources (encoding contributions from heterotic supergravity) (19) parameterized in the form (34) written in $\mathrm{N}$-adapted form as

$$
\begin{aligned}
& \widehat{R}_{1}^{1}=\widehat{R}_{2}^{2}=-{ }_{h} \Upsilon\left(x^{k}\right), \widehat{R}_{3}^{3}=\widehat{R}_{4}^{4}=-\Upsilon\left(x^{k}, y^{4}\right), \\
& \widehat{R}_{5}^{5}=\widehat{R}_{6}^{6}=-{ }_{1} \Upsilon\left(x^{k}, y^{a}, y^{6}\right), \\
& \widehat{R}_{7}^{7}=\widehat{R}_{8}^{8}=-{ }_{2} \Upsilon\left(x^{k}, y^{a}, y^{a_{1}}, y^{8}\right), \\
& \widehat{R}_{9}^{9}=\widehat{R}_{10}^{10}=-{ }_{3} \Upsilon\left(x^{k}, y^{a}, y^{a_{1}}, y^{a_{2}}, y^{10}\right) .
\end{aligned}
$$

Similar equations can be written recurrently for arbitrary finite extra dimensions.

\subsubsection{The ansatz for effective matter fields in heterotic string gravity}

Assumptions on nontrivial $\widehat{\phi}$-configurations We shall find dmetrics of type (5) for which the contributions of the field $\widehat{\phi}$ can be included by nonholonomic deformations $\stackrel{\circ}{\rightarrow} \rightarrow{ }^{s} \mathbf{g}$ into certain generic off-diagonal terms, i.e. into N-connection 
coefficients. For instance if under a prime configuration $0 \widehat{\phi}\left(x^{i}, y^{a}, y^{a_{s}}\right) \rightarrow \eta \widehat{\phi}=\widehat{\phi}\left(x^{i}, y^{4}\right)$ for which $\widehat{\mathbf{d}} \widehat{\phi}=0$. This equation is equivalent to a linear system of equations,

$\partial_{i} \widehat{\phi}-w_{i}\left(x^{k}, y^{4}\right) \widehat{\phi}^{*}=0$,

which can be solved in explicit form if the $\mathrm{N}$-connection coefficients $w_{i}$ are defined (see next sections for how such values can be found in explicit form). For such configurations, we state that ${ }^{\phi} \Upsilon_{\mu_{s} v_{s}}$ and ${ }^{\phi} \Lambda$ in (19) and (20) are zero and the fields $\widehat{\phi}$ with such configurations contribute to possible heterotic supergravity effects only via possible nontrivial off-diagonal interactions and not via effective sources. The terms with $\widehat{\phi}$ also vanish in all nonholonomic equations of motion (12)-(15) written with respect to $\mathrm{N}$-adapted frames if the conditions (36) are satisfied. Nontrivial coefficients with $\widehat{\phi}$ and its partial derivatives will appear if certain physical equations are written, for instance, in coordinate frames. We have chosen a special type of configurations for $\widehat{\phi}$ in order to simplify the procedure of finding generic off-diagonal solutions in heterotic string gravity in explicit form.

Nonholonomic gauge configurations In general form, we can consider any ${ }^{H} \Upsilon_{\mu_{s} v_{s}},{ }^{F} \Upsilon_{\mu_{s} v_{s}}$ and ${ }^{R} \Upsilon_{\mu_{s} v_{s}}$ for which $\Upsilon_{\mu_{s} v_{s}}={ }^{H} \Upsilon_{\mu_{s} v_{s}}+{ }^{F} \Upsilon_{\mu_{s} v_{s}}+{ }^{R} \Upsilon_{\mu_{s} v_{s}}$, can be nonholonomically transformed into an $\mathrm{N}$-adapted diagonal form

$$
\begin{aligned}
\Upsilon_{v_{s}}^{\mu_{s}}= & \operatorname{diag}\left[{ }_{h} \Upsilon\left(x^{k}\right),{ }_{h} \Upsilon\left(x^{k}\right), \Upsilon\left(x^{k}, y^{4}\right),\right. \\
& \Upsilon\left(x^{k}, y^{4}\right),{ }_{1} \Upsilon\left(x^{k}, y^{a}, y^{6}\right),{ }_{1} \Upsilon\left(x^{k}, y^{a}, y^{6}\right), \\
& 2 \Upsilon\left(x^{k}, y^{a}, y^{a_{1}}, y^{8}\right),{ }_{2} \Upsilon\left(x^{k}, y^{a}, y^{a_{1}}, y^{8}\right), \\
& \left.{ }_{3} \Upsilon\left(x^{k}, y^{a}, y^{a_{1}}, y^{a_{2}}, y^{10}\right),{ }_{3} \Upsilon\left(x^{k}, y^{a}, y^{a_{1}}, y^{a_{2}}, y^{10}\right)\right] .
\end{aligned}
$$

Nonholonomic deformations of string gauge fields can be parametrized in the form

$$
\begin{aligned}
& \widehat{\mathbf{H}}_{\alpha_{s} \beta_{s} \mu_{s}}={ }^{0} \widehat{\mathbf{H}}_{\alpha_{s} \beta_{s} \mu_{s}}+{ }^{\eta} \widehat{\mathbf{H}}_{\alpha_{s} \beta_{s} \mu_{s}}, \widehat{\mathbf{F}}_{\mu_{s} \alpha_{s}}={ }^{0} \widehat{\mathbf{F}}_{\mu_{s} \alpha_{s}} \\
&+{ }^{\eta} \widehat{\mathbf{F}}_{\mu_{s} \alpha_{s}}, \\
& \widetilde{\mathbf{R}}_{\mu_{s} \alpha_{s} \beta_{s} \gamma_{s}}={ }^{0} \widetilde{\mathbf{R}}_{\mu_{s} \alpha_{s} \beta_{s} \gamma_{s}}+{ }^{\eta} \widetilde{\mathbf{R}}_{\mu_{s} \alpha_{s} \beta_{s} \gamma_{s} .}
\end{aligned}
$$

where, respectively,

$$
\begin{aligned}
& { }^{H} \Upsilon_{v_{s}}^{\mu_{s}}=\operatorname{diag}\left[{ }_{h}^{H} \Upsilon\left(x^{k}\right),{ }_{h}^{H} \Upsilon\left(x^{k}\right),{ }^{H} \Upsilon\left(x^{k}, y^{4}\right),\right. \\
& { }^{H} \Upsilon\left(x^{k}, y^{4}\right),{ }_{1}^{H} \Upsilon\left(x^{k}, y^{a}, y^{6}\right),{ }_{1}^{H} \Upsilon\left(x^{k}, y^{a}, y^{6}\right), \\
& { }_{2}^{H} \Upsilon\left(x^{k}, y^{a}, y^{a_{1}}, y^{8}\right),{ }_{2}^{H} \Upsilon\left(x^{k}, y^{a}, y^{a_{1}}, y^{8}\right) \text {, } \\
& { }_{3}^{H} \Upsilon\left(x^{k}, y^{a}, y^{a_{1}}, y^{a_{2}}, y^{10}\right) \text {, } \\
& \left.{ }_{3}^{H} \Upsilon\left(x^{k}, y^{a}, y^{a_{1}}, y^{a_{2}}, y^{10}\right)\right] \text {; } \\
& { }^{F} \Upsilon{ }_{v_{s}}^{\mu_{s}}=\operatorname{diag}\left[{ }_{h}^{F} \Upsilon\left(x^{k}\right),{ }_{h}^{F} \Upsilon\left(x^{k}\right),{ }^{F} \Upsilon\left(x^{k}, y^{4}\right),\right. \\
& { }^{F} \Upsilon\left(x^{k}, y^{4}\right),{ }_{1}^{F} \Upsilon\left(x^{k}, y^{a}, y^{6}\right),{ }_{1}^{F} \Upsilon\left(x^{k}, y^{a}, y^{6}\right), \\
& { }_{2}^{F} \Upsilon\left(x^{k}, y^{a}, y^{a_{1}}, y^{8}\right),{ }_{2}^{F} \Upsilon\left(x^{k}, y^{a}, y^{a_{1}}, y^{8}\right) \text {, } \\
& { }_{3}^{F} \Upsilon\left(x^{k}, y^{a}, y^{a_{1}}, y^{a_{2}}, y^{10}\right) \text {, } \\
& \left.{ }_{3}^{F} \Upsilon\left(x^{k}, y^{a}, y^{a_{1}}, y^{a_{2}}, y^{10}\right)\right] \text {; } \\
& { }^{R} \Upsilon{ }_{v_{s}}^{\mu_{s}}=\operatorname{diag}\left[{ }_{h}^{R} \Upsilon\left(x^{k}\right),{ }_{h}^{R} \Upsilon\left(x^{k}\right),{ }^{R} \Upsilon\left(x^{k}, y^{4}\right),\right. \\
& { }^{R} \Upsilon\left(x^{k}, y^{4}\right),{ }_{1}^{R} \Upsilon\left(x^{k}, y^{a}, y^{6}\right),{ }_{1}^{R} \Upsilon\left(x^{k}, y^{a}, y^{6}\right),
\end{aligned}
$$

$$
\begin{aligned}
& { }_{2}^{R} \Upsilon\left(x^{k}, y^{a}, y^{a_{1}}, y^{8}\right),{ }_{2}^{R} \Upsilon\left(x^{k}, y^{a}, y^{a_{1}}, y^{8}\right), \\
& { }_{3}^{R} \Upsilon\left(x^{k}, y^{a}, y^{a_{1}}, y^{a_{2}}, y^{10}\right), \\
& \left.{ }_{3}^{R} \Upsilon\left(x^{k}, y^{a}, y^{a_{1}}, y^{a_{2}}, y^{10}\right)\right] .
\end{aligned}
$$

For instance, kink-like almost-Kähler configurations can be encoded as a prime configuration ${ }^{0} \widehat{\mathbf{H}}_{\alpha_{s} \beta_{s} \mu_{s}}$ modifying for the target configuration $\widehat{\mathbf{H}}_{\alpha_{s} \beta_{s} \mu_{s}}$ the solutions for off-diagonal metrics via an effective source.

Such parametrizations are possible by considering the ansatz with effective cosmological constants

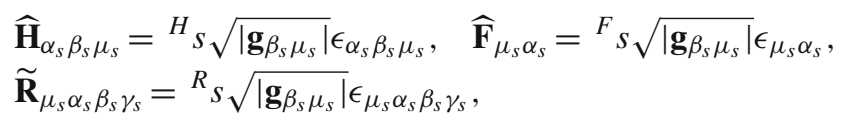

with absolute antisymmetric $\epsilon$-tensors (we refer the reader to similar details for the 4-d case to [42] and the references therein). For such an ansatz, we obtain effective energymomentum tensors

$$
\begin{aligned}
& { }^{H} \Upsilon_{\mu_{s} v_{s}}=-\frac{10}{2}\left({ }^{H} s\right)^{2} \mathbf{g}_{\beta_{s} \mu_{s}}, \text { for }{ }^{H} \Lambda=-5\left({ }^{H} s\right)^{2}, \\
& { }^{F} \Upsilon_{\mu_{s} v_{s}}=-\frac{10 \alpha^{\prime}}{2} n_{F}\left({ }^{F} s\right)^{2} \mathbf{g}_{\beta_{s} \mu_{s}}, \text { for }{ }^{F} \Lambda=-5 n_{F}\left({ }^{F} s\right)^{2}, \\
& { }^{R} \Upsilon_{\mu_{s} v_{s}}=\frac{10 \alpha^{\prime}}{2} n_{R}\left({ }^{R} s\right)^{2} \mathbf{g}_{\beta_{s} \mu_{s}}, \text { for }{ }^{R} \Lambda=-5 \operatorname{tr} n_{R}\left({ }^{R} s\right)^{2},
\end{aligned}
$$

were we considered Eqs. (34) and (19)-(23) and, for instance the numbers $n_{F}=\operatorname{tr}\left[\right.$ internal $F$ ] and $n_{R}=\operatorname{tr}[$ internal $\widetilde{R}$ ] depends on the representation of the Lie algebra for $F$ and on the representation of Lie groups on the internal space.

\subsubsection{Decoupling of nonholonomic equations of motion and effective}

Considering the ansatz (24) for $g_{i}\left(x^{k}\right)=\epsilon_{i} e^{q\left(x^{k}\right)}, \epsilon_{i}=1$, in (35) with respective sources, we obtain this nonlinear system of PDEs:

$q^{*}+q^{\prime \prime}=2{ }_{h} \Upsilon$,

$\varpi^{*} h_{3}^{*}=2 h_{3} h_{4} \Upsilon$,

$n_{i}^{* *}+\gamma n_{i}^{*}=0$,

$\beta w_{i}-\alpha_{i}=0$,

${ }^{1} \varpi^{*_{1}} h_{5}^{{ }^{*} 1}=2 h_{5} h_{61} \Upsilon$,

${ }^{1} n_{i_{1}}^{*_{1} *_{1}}+{ }^{1} \gamma^{1} n_{i_{1}}^{*_{1}}=0$,

${ }^{1} \beta^{1} w_{i_{1}}-{ }^{1} \alpha_{1}=0$,

${ }^{2} \varpi^{* 2} h_{7}^{* 2}=2 h_{7} h_{8} \Upsilon$ ,

${ }^{2} n_{i_{2}}^{* 2 * 2}+{ }^{2} \gamma^{2} n_{i_{2}}^{* 2}=0$,

${ }^{2} \beta^{2} w_{i_{2}}-{ }^{2} \alpha_{i_{2}}=0$,

${ }^{3} \varpi^{* 3} h_{7}^{* 3}=2 h_{9} h_{103} \Upsilon$,

${ }^{3} n_{i_{3}{ }^{*_{3} *_{3}}}+{ }^{3} \gamma^{3} n_{i_{3}}^{*_{3}}=0$,

${ }^{3} \beta^{3} w_{i_{3}}-{ }^{3} \alpha_{i_{3}}=0$. 
In equivalent form, the same equations are obtained recurrently if we write, for instance, ${ }^{s} w_{i_{s}}$ instead of ${ }^{s} w_{\tau_{s-1}},{ }^{s} n_{i_{s}}$ instead of ${ }^{s} n_{\tau_{s-1}}$ etc. (some equations and solutions can be parametrized in more simple forms if we follow the first convention, other equations will be more "compact" if we follow the second convention). In these equations, the generating functions

$$
\begin{aligned}
& \varpi=\ln |\Phi|, \quad{ }^{1} \varpi=\ln \left|\quad{ }^{1} \Phi\right|, \quad{ }^{2} \varpi=\ln \left|{ }^{2} \Phi\right|, \\
& { }^{3} \varpi=\left.\ln \right|^{3} \Phi \mid,
\end{aligned}
$$

(we shall use this in the next sections, on convenience formulas with $\varpi$ - and/or $\Phi$-values) and the $\alpha$-, $\beta$-, $\gamma$-coefficients on corresponding shells are defined, respectively:

$$
\begin{aligned}
& \varpi=\ln \left|\frac{h_{3}^{*}}{\sqrt{\left|h_{3} h_{4}\right|}}\right|, \quad \gamma:=\left(\ln \frac{\left|h_{3}\right|^{3 / 2}}{\left|h_{4}\right|}\right)^{*}, \\
& \alpha_{i}=\frac{h_{3}^{*}}{2 h_{3}} \partial_{i} \varpi, \quad \beta=\frac{h_{3}^{*}}{2 h_{3}} \varpi^{*}, \\
& { }^{1} \varpi=\ln \left|\frac{h_{5}^{* 1}}{\sqrt{\left|h_{5} h_{6}\right|}}\right|, \quad{ }^{1} \gamma:=\left(\ln \frac{\left|h_{5}\right|^{3 / 2}}{\left|h_{6}\right|}\right)^{*_{1}}, \\
& { }^{1} \alpha_{\tau}=\frac{h_{5}^{* 1}}{2 h_{5}} \partial_{\tau}{ }^{1} \varpi, \quad{ }^{1} \beta=\frac{h_{5}^{* 1}}{2 h_{5}} \partial_{\tau}{ }^{1} \varpi, \\
& { }^{2} \varpi=\ln \left|\frac{h_{7}^{* 2}}{\sqrt{\left|h_{7} h_{8}\right|}}\right|, \quad{ }^{2} \gamma:=\left(\ln \frac{\left|h_{7}\right|^{3 / 2}}{\left|h_{8}\right|}\right)^{* 2}, \\
& { }^{2} \alpha_{\tau_{1}}=\frac{h_{7}^{* 2}}{2 h_{7}} \partial_{\tau_{1}}{ }^{2} \varpi, \quad{ }^{2} \beta=\frac{h_{7}^{* 2}}{2 h_{7}} \partial_{\tau_{1}}{ }^{2} \varpi, \\
& { }^{3} \varpi=\ln \left|\frac{h_{9}^{* 3}}{\sqrt{\left|h_{7} h_{8}\right|}}\right|, \quad{ }^{3} \gamma:=\left(\ln ^{3} \frac{\left|h_{9}\right|^{3 / 2}}{\left|h_{10}\right|}\right)^{* 3}, \\
& { }^{3} \alpha_{\tau_{2}}=\frac{h_{9}^{* 3}}{2 h_{9}} \partial_{\tau_{2}}{ }^{3} \varpi, \quad{ }^{3} \beta=\frac{h_{9}^{* 3}}{2 h_{9}} \partial_{\tau_{2}}{ }^{3} \varpi,
\end{aligned}
$$

when the frame/coordinate systems are chosen in such a way that nonzero conditions for the partial derivatives are satisfied: $\varpi^{*}, h_{a}^{*},{ }^{1} \varpi^{*_{1}}, h_{a_{1}}^{* 1},{ }^{2} \varpi^{*_{2}}, h_{a_{2}}^{* 2},{ }^{3} \varpi^{*_{3}}, h_{a_{3}}^{* 3} \neq 0$ (this allows us to avoid singular nonholonomic deformations).

Equations (39)-(47) prove a very important decoupling property of the heterotic string equations (12) and $4 d-10 d$ (generalized) Einstein equations (16) with respect to correspondingly $\mathrm{N}$-adapted frames. In explicit and in certain simple forms, such formulas can be obtained for metrics with at least one Killing symmetry. Nevertheless, the constructions can be generalized for non-Killing configurations in any finite extra-dimension gravity; see a corresponding technique in $[40,43]$. In brief the decoupling property of the AFDM can be explained for the 4-d configurations:

1. Equation (39) is just a 2-d Laplacian, which can be solved for any $h$-source ${ }_{h} \Upsilon\left(x^{k}\right)$.

2. Equation (40) contains only the partial derivative $\partial_{4}$, equivalently $*$-derivative, and constraints by a system of two equations, together with the formula for the value $\varpi$ in (48), four values $h_{3}\left(x^{i}, y^{4}\right), h_{4}\left(x^{i}, y^{4}\right)$ and $\varpi\left(x^{i}, y^{4}\right)$ and source $\Upsilon\left(x^{k}, y^{4}\right)$. Prescribing any two such functions, we can define (integrating on $y^{4}$ ) two other such functions. We note that $h_{a}$ are coefficients of a d-metric, $\varpi$ is a generating function, and $\Upsilon$ is related to extradimensional string contributions.

3 . Using $h_{3}$ and $\varpi$, or $\Phi$, from the previous point, we compute the coefficients $\alpha_{i}$ and $\beta$; see (48). This allows us to define $w_{i}$ from the algebraic equations (42).

4. Having computed the coefficient $\gamma$ (48), the N-connection coefficients $n_{i}$ can be defined after two integrations on $y^{4}$ in (41).

We can repeat the steps 2-4 recurrently on shells $s=$ $1,2,3$, adding dependencies on $2+2+2$ extra-dimension coordinates, respectively. In this way, we involve new classes of generating functions and effective sources. Solving the systems (43)-(47) recurrently, we can compute all d-metric and $\mathrm{N}$-connection coefficients for a 10-d ansatz (5).

\subsubsection{Integration of nonholonomic equations of motion by generating functions and effective sources}

The system of nonlinear PDEs (39)-(47) with coefficients (48) and effective sources (37) with contributions from string gravity can be integrated in general forms on any shell up to 10-d.

4- $d$ non-vacuum configurations The coefficients $g_{i}=e^{q\left(x^{k}\right)}$ are defined by solutions of the corresponding 2-d Poisson equation (39) as we mentioned above (see point 1 at the end of previous subsection).

We can integrate the system of nonlinear PDEs consisting of the first equation in (48) and (40) for arbitrary source $\Upsilon\left(x^{k}, y^{4}\right)$ and with generating function $\Phi\left(x^{k}, y^{4}\right)=e^{\varpi}$. The solutions will be generated for a stationary d-metric when the coefficients do not depend on time-like coordinate $y^{3}=t$, when $\Phi^{*} \neq 0$. The vertical effective gravitational field equations (40)-(42) transform, respectively, into

$$
\begin{aligned}
& \Phi^{*} h_{3}^{*}=2 h_{3} h_{4} \Upsilon \Phi, \\
& \sqrt{\left|h_{3} h_{4}\right|} \Phi=h_{3}^{*}, \\
& n_{i}^{* *}+\left(\ln \left|h_{3}\right|^{3 / 2} /\left|h_{4}\right|\right)^{*} n_{i}^{*}=0, \\
& \Phi^{*} w_{i}-\partial_{i} \Phi=0 .
\end{aligned}
$$

This system can be solved in very general forms by prescribing $\Upsilon$ and $\Phi$ and integrating the equations "step by step". Introducing the function

$\rho^{2}:=-h_{3} h_{4}$

(the sign - is motivated by the pseudo-Euclidean signature), we express (49) and (50) as

$\Phi^{*} h_{3}^{*}=-2 \rho^{2} \Upsilon \Phi$ and $h_{3}^{*}=\rho \Phi$. 
Using $h_{3}^{*}$ from the second equation (54) in the first equation, we write

$\rho=-\frac{1}{2} \frac{\Phi^{*}}{\Upsilon}$.

This value, together with the second equation of (54) and a further integration on $y^{4}$, result in

$$
h_{3}=h_{3}^{[0]}\left(x^{k}\right)-\frac{1}{4} \int \mathrm{d} y^{4} \frac{\left(\Phi^{2}\right)^{*}}{\Upsilon},
$$

where $h_{3}^{[0]}\left(x^{k}\right)$ is an integration function. Considering Eqs. (55), (53), and (56), we compute

$$
\begin{aligned}
h_{4} & =-\frac{1}{4 h_{3}}\left(\frac{\Phi^{*}}{\Upsilon}\right)^{2} \\
& =-\frac{1}{4}\left(\frac{\Phi^{*}}{\Upsilon}\right)^{2}\left(h_{3}^{[0]}-\frac{1}{4} \int \mathrm{d} y^{4} \frac{\left(\Phi^{2}\right)^{*}}{\Upsilon}\right)^{-1} .
\end{aligned}
$$

The first parts of the N-connection coefficients are found by integrating two times on $y^{4}$ in (51) written in the form

$$
n_{i}^{* *}=\left(n_{i}^{*}\right)^{*}=-n_{i}^{*}\left(\ln \left|h_{3}\right|^{3 / 2} /\left|h_{4}\right|\right)^{*}
$$

for the coefficient $\gamma$ defined in (48). Integrating two times on $y^{4}$ for explicit values of (57) and (56), we compute

$$
\begin{aligned}
n_{k}\left(x^{i}, y^{4}\right)= & { }_{1} n_{k}+{ }_{2} n_{k} \int \mathrm{d} y^{4} \frac{h_{4}}{\left|h_{3}\right|^{3 / 2}} \\
= & { }_{1} n_{k}+{ }_{2} \tilde{n}_{k} \int \mathrm{d} y^{4} \frac{\left(\Phi^{2}\right)^{*}}{\left|h_{3}\right|^{5 / 2} \Upsilon^{2}} \\
= & { }_{1} n_{k}+{ }_{2} n_{k} \int \mathrm{d} y^{4} \frac{\left(\Phi^{2}\right)^{*}}{\Upsilon^{2}} \\
& \times\left|h_{3}^{[0]}-\frac{1}{4} \int \mathrm{d} y^{4} \frac{\left(\Phi^{*}\right)^{2}}{\Upsilon}\right|^{-5 / 2},
\end{aligned}
$$

containing also a second set of integration functions ${ }_{1} n_{k}\left(x^{i}\right)$ and the redefined second integration function ${ }_{2} \widetilde{n}_{k}\left(x^{i}\right)$.

We can solve the linear algebraic equations (52) and express

$w_{i}=\partial_{i} \Phi / \Phi^{*}$.

Putting together all the above formulas and writing the effective source (34) in explicit form, we obtain the formulas for the coefficients of a d-metric and a N-connection determining a class of stationary solutions for the system (39)(42),

$$
g_{i}=g_{i}\left[q,{ }_{h} \Upsilon,\right]=e^{q\left(x^{k}\right)}
$$

as a solution of 2-d Poisson equations (39);

$$
\begin{aligned}
& h_{3}=h_{3}^{[0]}\left(x^{k}\right)-\frac{1}{4} \int \mathrm{d} y^{4} \frac{\left(\Phi^{2}\right)^{*}}{\Upsilon} ; \\
& h_{4}=-\frac{1}{4}\left(\frac{\Phi^{*}}{\Upsilon}\right)^{2}\left(h_{3}^{[0]}-\frac{1}{4} \int \mathrm{d} y^{4} \frac{\left(\Phi^{2}\right)^{*}}{\Upsilon}\right)^{-1} ;
\end{aligned}
$$

$n_{k}={ }_{1} n_{k}+{ }_{2} n_{k} \int \mathrm{d} y^{4} \frac{\left(\Phi^{2}\right)^{*}}{\Upsilon^{2}}\left|h_{3}^{[0]}-\frac{1}{4} \int \mathrm{d} y^{4} \frac{\left(\Phi^{2}\right)^{*}}{\Upsilon}\right|^{-5 / 2} ;$

$w_{i}=\partial_{i} \Phi / \Phi^{*}$.

Using coefficients (58), we define such a class of quadratic elements for off-diagonal 4-d stationary configurations in heterotic supergravity with nonholonomically induced torsion,

$$
\begin{aligned}
\mathrm{d} s_{K 4 d}^{2}= & g_{\alpha \beta}\left(x^{k}, y^{4}\right) \mathrm{d} u^{\alpha} \mathrm{d} u^{\beta}=e^{q}\left[\left(\mathrm{~d} x^{1}\right)^{2}+\left(\mathrm{d} x^{2}\right)^{2}\right] \\
& +\left[h_{3}^{[0]}\left(x^{k}\right)-\frac{1}{4} \int \mathrm{d} y^{4} \frac{\left(\Phi^{2}\right)^{*}}{\Upsilon}\right] \\
& \times\left[\mathrm{d} t+\left({ }_{1} n_{k}+{ }_{2} n_{k} \int \mathrm{d} y^{4} \frac{\left(\Phi^{2}\right)^{*}}{\Upsilon^{2}}\right.\right. \\
& \left.\left.\times\left|h_{3}^{[0]}-\frac{1}{4} \int \mathrm{d} y^{4} \frac{\left(\Phi^{2}\right)^{*}}{\Upsilon}\right|^{-5 / 2}\right) \mathrm{~d} x^{k}\right]^{2} \\
& -\frac{1}{4}\left(\frac{\Phi^{*}}{\Upsilon}\right)^{2}\left(h_{3}^{[0]}-\frac{1}{4} \int \mathrm{d} y^{4} \frac{\left(\Phi^{2}\right)^{*}}{\Upsilon}\right)^{-1} \\
& \times\left[\mathrm{d} y^{4}+\frac{\partial_{i} \Phi}{\Phi^{*}} \mathrm{~d} x^{i}\right]^{2} .
\end{aligned}
$$

Such a class of metrics also contains exact solutions for the canonical d-connection $\widehat{\mathbf{D}}$ in $R^{2}$ gravity with effective scalar field encoded into a nonholonomically polarized vacuum with a special parametrization of the source $\Upsilon$; see for details $[14,70]$.

Examples of 4-d-10-vacuum configurations The configurations with zero source cannot be constructed as particular cases of former off-diagonal solutions with $\Upsilon=0$ because these limits are not smooth for metrics (59). In string heterotic gravity, such conditions can be satisfied if different effective fields compensate their mutual contributions and result in an effective vacuum gravitational configuration.

For the ansatz (24), we can construct solutions when the nontrivial coefficients of the Ricci d-tensor (25)-(32) are zero but the Ricci and torsion d-tensor are not trivial. The first equation is a typical example of 2-d Laplace equation with solutions expressed in the form $g_{i}=e^{q\left(x^{k}, \Upsilon=0\right)}$.

In 4-d, there are three general classes of off-diagonal metrics which result in zero coefficients (26)-(28). Such constructions can be generalized for 10-d generic off-diagonals with mixing standard vacuum configurations for the 4-d spacetimes.

1. If $h_{3}^{*}=0$ but $h_{3} \neq 0, h_{4}^{*} \neq 0$ and $h_{4} \neq 0$, we obtain only one nontrivial equation (27),

$$
n_{k}^{* *}+n_{k}^{*}\left(\ln \left|h_{4}\right|\right)^{*}=0
$$


where $h_{4}\left(x^{i}, y^{4}\right)$ and $w_{k}\left(x^{i}, y^{4}\right)$ are arbitrary generating functions. Integrating two times on $y^{4}$, we obtain

$n_{k}={ }_{1} n_{k}+{ }_{2} n_{k} \int \mathrm{d} y^{4} / h_{4}$

with integration functions ${ }_{1} n_{k}\left(x^{i}\right)$ and ${ }_{2} n_{k}\left(x^{i}\right)$. In 4-d, this defines a quadratic line element

$$
\begin{aligned}
\mathrm{d} s_{v, 4-d}^{2} & \\
= & e^{q\left(x^{k}, \Upsilon=0\right)}\left(\mathrm{d} x^{i}\right)^{2}+{ }^{0} h_{3}\left(x^{k}\right) \\
& \times\left[\mathrm{d} t+\left({ }_{1} n_{k}\left(x^{i}\right)+{ }_{2} n_{k}\left(x^{i}\right) \int \mathrm{d} y^{4} / h_{4}\right) \mathrm{d} x^{k}\right]^{2} \\
& +h_{4}\left(x^{i}, y^{4}\right)\left[\mathrm{d} y^{4}+w_{i}\left(x^{k}, y^{4}\right) \mathrm{d} x^{i}\right]^{2} .
\end{aligned}
$$

Recurrently, we can construct effective stationary vacuum configurations in 10-d [with $i_{1}=(1,2,3,4) ; i_{2}=$ $(1,2,3,4,5,6) ; i_{3}=(1,2,3,4,5,6,7,8) ; a_{1}=5,6$; $\left.a_{2}=7,8 ; a_{3}=9,10\right]$,

$$
\begin{aligned}
& \mathrm{d} s_{v, 10-d}^{2}=e^{q\left(x^{k}, \Upsilon=0\right)}\left(\mathrm{d} x^{i}\right)^{2} \\
& +\left\{{ }^{0} h_{3}\left(x^{k}\right)\left[\mathrm{d} t+\left({ }_{1} n_{k}\left(x^{i}\right)+{ }_{2} n_{k}\left(x^{i}\right) \int \mathrm{d} y^{4} / h_{4}\right) \mathrm{d} x^{k}\right]^{2}\right. \\
& \left.+h_{4}\left(x^{i}, y^{4}\right)\left[\mathrm{d} y^{4}+w_{i}\left(x^{k}, y^{4}\right) \mathrm{d} x^{i}\right]^{2}\right\}_{\mathrm{type} 1, s=0} \\
& +{ }^{0} h_{5}\left(x^{k}, y^{4}\right)\left[\mathrm{d} y^{5}+\left({ }_{1} n_{k_{1}}\left(x^{i}, y^{4}\right)\right.\right. \\
& \left.\left.\left.+{ }_{2}^{1} n_{k_{1}}\left(x^{i}, y^{6}\right) \int \mathrm{d} y^{6} / h_{6}\right) \mathrm{~d} x^{k_{1}}\right]^{2}\right\} \\
& +\left\{h_{6}\left(x^{i}, y^{4}, y^{6}\right)\left[\mathrm{d} y^{6}+{ }^{1} w_{i_{1}}\left(x^{k}, y^{4}, y^{6}\right) \mathrm{d} x^{i_{1}}\right]^{2}\right. \\
& +{ }^{0} h_{7}\left(x^{k}, y^{4}, y^{a_{1}}\right)\left[\mathrm{d} y^{7}+\left({ }_{1}^{2} n_{k_{2}}\left(x^{i}, y^{4}, y^{a_{1}}\right)\right.\right. \\
& \left.\left.\left.+{ }_{2}^{2} n_{k_{2}}\left(x^{i}, y^{4}, y^{a_{1}}\right) \int \mathrm{d} y^{8} / h_{8}\right) \mathrm{~d} x^{k_{2}}\right]^{2}\right\} \\
& +\left\{h_{8}\left(x^{i}, y^{4}, y^{a_{1}}, y^{8}\right)\left[\mathrm{d} y^{8}+{ }^{2} w_{i_{2}}\left(x^{k}, y^{4}, y^{a_{1}}, y^{8}\right) \mathrm{d} x^{i_{2}}\right]^{2}\right. \\
& +{ }^{0} h_{9}\left(x^{k}, y^{4}, y^{a_{1}}, y^{a_{2}}\right)\left[\mathrm{d} y^{9}+\left({ }_{1}^{3} n_{k_{3}}\left(x^{i}, y^{4}, y^{a_{1}}, y^{a_{2}}\right)\right.\right. \\
& \left.\left.+{ }_{2}^{3} n_{k_{3}}\left(x^{i}, y^{4}, y^{a_{1}}, y^{a_{2}}\right) \int \mathrm{d} y^{10} / h_{10}\right) \mathrm{~d} x^{k_{3}}\right]^{2} \\
& +h_{10}\left(x^{i}, y^{4}, y^{a_{1}}, y^{a_{2}}, y^{10}\right)\left[\mathrm{d} y^{10}\right. \\
& \left.\left.+{ }^{3} w_{i_{3}}\left(x^{k}, y^{4}, y^{a_{1}}, y^{a_{2}}, y^{10}\right) \mathrm{d} x^{i_{3}}\right]^{2}\right\} \\
& \text { type } 1, s=3
\end{aligned}
$$

For instance, the bracket $\{\ldots\}_{\text {type } 1, s=2}$ states that the vacuum metric is of type 1 on shell 2 . The coefficients of such d-metrics do not depend on variables $\left(t, y^{9}\right)$ and, on respective shells, the values

$$
\begin{aligned}
& { }^{0} h_{3},{ }_{1} n_{k},{ }_{2} n_{k},{ }^{0} h_{5},{ }_{1}^{1} n_{k_{1}},{ }_{2}^{1} n_{k_{1}},{ }^{0} h_{7},{ }_{1}^{2} n_{k_{2}},{ }_{2}^{2} n_{k_{2}}, \\
& { }^{0} h_{9},{ }_{1}^{3} n_{k_{3}},{ }_{2}^{3} n_{k_{3}}
\end{aligned}
$$

are integration functions and $q, h_{4}, w_{i}, h_{6},{ }^{1} w_{i_{1}}, h_{8}$, ${ }^{2} w_{i_{2}}, h_{10},{ }^{3} w_{i_{3}}$ are generating functions for 10 -d nonholonomic effective vacuum heterotic string configurations. In the above formulas, $h_{5}^{*_{1}}=0, h_{5} \neq 0, h_{6}^{*_{1}} \neq 0$, and $h_{6} \neq 0 ; h_{7}^{* 2}=0, h_{7} \neq 0, h_{8}^{* 2} \neq 0$, and $h_{10} \neq 0 ; h_{9}^{* 3}=0, h_{9} \neq 0, h_{10}^{* 3} \neq 0$, and $h_{10} \neq 0$.

2. In such cases, $h_{3}^{*} \neq 0$ and $h_{4}^{*} \neq 0$. It is possible to solve the Eqs. (26) and (40) in a self-consistent form for $\Upsilon=$ 0 if $\varpi^{*}=0$ for respective coefficients in (48). Fixing $\varpi=\varpi_{0}=$ const, we can consider arbitrary functions $w_{i}\left(x^{k}, y^{4}\right)$ because $\beta=\alpha_{i}=0$ for such configurations. The conditions (48) are satisfied by any

$h_{4}={ }^{0} h_{4}\left(x^{k}\right)\left[\left(\sqrt{\left|h_{3}\right|}\right)^{*}\right]^{2}$,

where ${ }^{0} h_{3}\left(x^{k}\right)$ is an integration function and $h_{3}\left(x^{k}, y^{4}\right)$ is any generating function. The coefficients $n_{k}$ should be found from (27); see (60). Such a family of 4-d vacuum generic off-diagonal metrics is described by

$$
\begin{aligned}
\mathrm{d} s_{v, 4 d}^{2}= & e^{q\left(x^{k}, \Upsilon=0\right)}\left(\mathrm{d} x^{i}\right)^{2}+h_{3}\left(x^{i}, y^{4}\right)\left\{\mathrm{d} t+\left({ }_{1} n_{k}\left(x^{i}\right)\right.\right. \\
& \left.+{ }_{2} \widetilde{n}_{k}\left(x^{i}\right) \int \mathrm{d} y^{4}\left[\left(\left|h_{3}\right|^{3 / 4}\right)^{*}\right]^{2} \mathrm{~d} x^{i}\right\}^{2} \\
& +{ }^{0} h_{4}\left(x^{k}\right)\left[\left(\sqrt{\left|h_{3}\right|}\right)^{*}\right]^{2}\left[\mathrm{~d} y^{4}+w_{i}\left(x^{k}, y^{4}\right) \mathrm{d} x^{i}\right]^{2},
\end{aligned}
$$

where the integration function ${ }_{2} \widetilde{n}_{k}\left(x^{i}\right)$ contains certain integration coefficients. We can extend such metrics on any shell $s=1,2,3$ preserving the conditions of a zero effective source and adding respective generating functions

$$
\begin{aligned}
& s=1:{ }^{1} \varpi^{* 1}=0, h_{6}={ }^{0} h_{6}\left(x^{k}, y^{4}\right)\left[\left(\sqrt{\left|h_{5}\right|}\right)^{*_{1}}\right]^{2}, \\
& \text { gener. functs. }\left\{\begin{array}{c}
h_{5}\left(x^{i}, y^{4}, y^{6}\right) \\
{ }^{1} w_{i_{1}}\left(x^{i}, y^{4}, y^{6}\right)
\end{array} ;\right. \\
& s=2:{ }^{2} \varpi^{*_{2}}=0, h_{8}={ }^{0} h_{8}\left(x^{k}, y^{4}, y^{a_{1}}\right)\left[\left(\sqrt{\left|h_{7}\right|}\right)^{*_{2}}\right]^{2}, \\
& \text { gener. functs. }\left\{\begin{array}{c}
h_{7}\left(x^{i}, y^{4}, y^{a_{1}}, y^{8}\right) \\
{ }^{2} w_{i_{2}}\left(x^{i}, y^{4}, y^{a_{1}}, y^{8}\right) ;
\end{array}\right. \\
& s=3:{ }^{3} \varpi^{* 3}=0, h_{10}={ }^{0} h_{10}\left(x^{k}, y^{4}, y^{a_{1}}, y^{a_{2}}\right)\left[\left(\sqrt{\left|h_{9}\right|}\right)^{* 3}\right]^{2}, \\
& \text { gener. functs. }\left\{\begin{array}{c}
h_{9}\left(x^{i}, y^{4}, y^{a_{1}}, y^{a_{2}}, y^{10}\right) \\
{ }^{3} w_{i_{3}}\left(x^{i}, y^{4}, y^{a_{1}}, y^{a_{2}}, y^{10}\right) .
\end{array}\right.
\end{aligned}
$$


In 10-d, the vacuum solutions (62) are generalized to

$$
\begin{aligned}
& \mathrm{d} s_{v, 10-d}^{2}=e^{q}\left(\mathrm{~d} x^{i}\right)^{2} \\
& +h_{3}\left\{\mathrm{~d} t+\left({ }_{1} n_{k}+{ }_{2} \tilde{n}_{k} \int \mathrm{d} y^{4}\left[\left(\left|h_{3}\right|^{3 / 4}\right)^{*}\right]^{2} \mathrm{~d} x^{k}\right\}^{2}\right. \\
& +{ }^{0} h_{4}\left[\left(\sqrt{\left|h_{3}\right|}\right)^{*}\right]^{2}\left[\mathrm{~d} y^{4}+w_{i} \mathrm{~d} x^{i}\right]^{2} \\
& +h_{5}\left\{\mathrm{~d} y^{5}+\left({ }_{1}^{1} n_{k_{1}}+{ }_{2} \widetilde{n}_{k_{1}} \int \mathrm{d} y^{6}\left[\left(\left|h_{5}\right|^{3 / 4}\right)^{*_{1}}\right]^{2} \mathrm{~d} x^{k_{1}}\right\}^{2}\right. \\
& +{ }^{0} h_{6}\left[\left(\sqrt{\left|h_{5}\right|}\right)^{* 1}\right]^{2}\left[\mathrm{~d} y^{6}+{ }^{1} w_{i_{1}} \mathrm{~d} x^{i_{1}}\right]^{2} \\
& +h_{7}\left\{\mathrm{~d} y^{7}+\left({ }_{1}^{2} n_{k_{2}}+{ }_{2} \widetilde{n}_{k_{2}} \int \mathrm{d} y^{8}\left[\left(\left|h_{7}\right|^{3 / 4}\right)^{* 2}\right]^{2} \mathrm{~d} x^{k_{2}}\right\}^{2}\right. \\
& +{ }^{0} h_{8}\left[\left(\sqrt{\left|h_{7}\right|}\right)^{*_{2}}\right]^{2}\left[\mathrm{~d} y^{8}+{ }^{2} w_{i_{2}} \mathrm{~d} x^{i_{2}}\right]^{2} \\
& +h_{9}\left\{\mathrm{~d} y^{9}+\left({ }_{1}^{3} n_{k_{3}}+{ }_{2} \widetilde{n}_{k_{3}} \int \mathrm{d} y^{10}\left[\left(\left|h_{9}\right|^{3 / 4}\right)^{*_{3}}\right]^{2} \mathrm{~d} x^{k_{3}}\right\}^{2}\right. \\
& +{ }^{0} h_{10}\left[\left(\sqrt{\left|h_{9}\right|}\right)^{* 3}\right]^{2}\left[\mathrm{~d} y^{10}+{ }^{2} w_{i_{3}} \mathrm{~d} x^{i_{3}}\right]^{2},
\end{aligned}
$$

for integration functions

$$
\begin{aligned}
& { }^{0} h_{4}\left(x^{k}\right), 1, n_{k}\left(x^{i}\right),{ }_{2} \widetilde{n}_{k}\left(x^{i}\right) ;{ }^{0} h_{6}\left(x^{k}, y^{4}\right), \\
& \quad 1 n_{k_{1}}\left(x^{i}, y^{4}\right),{ }_{2} \widetilde{n}_{k_{1}}\left(x^{i}, y^{4}\right) ; \\
& { }^{0} h_{8}\left(x^{k}, y^{4}, y^{a_{1}}\right),{ }_{1}^{2} n_{k_{2}}\left(x^{i}, y^{4}, y^{a_{1}}\right), \\
& \quad{ }_{2}^{2} \widetilde{n}_{k_{2}}\left(x^{i}, y^{4}, y^{a_{1}}\right) ; \\
& { }^{0} h_{10}\left(x^{k}, y^{4}, y^{a_{1}}, y^{a_{2}}\right),{ }_{1}^{3} n_{k_{3}}\left(x^{i}, y^{4}, y^{a_{1}}, y^{a_{2}}\right), \\
& { }_{2}^{3} \widetilde{n}_{k_{2}}\left(x^{i}, y^{4}, y^{a_{1}}, y^{a_{2}}\right),
\end{aligned}
$$

In the above shell formulas, $h_{5}^{*_{1}} \neq 0$ and $h_{6}^{*_{1}} \neq 0 ; h_{7}^{*_{2}} \neq$ 0 and $h_{8}^{* 2} \neq 0 ; h_{9}^{* 3} \neq 0$ and $h_{10}^{* 3} \neq 0$.

3. We consider that $h_{3}^{*} \neq 0$ but $h_{4}^{*}=0$. Equation (26) transforms into

$$
h_{3}^{* *}-\frac{\left(h_{3}^{*}\right)^{2}}{2 h_{3}}=0
$$

when the general solution is $h_{3}\left(x^{k}, y^{4}\right)=\left[c_{1}\left(x^{k}\right)+c_{2}\right.$ $\left.\left(x^{k}\right) y^{4}\right]^{2}$, with generating functions $c_{1}\left(x^{k}\right), c_{2}\left(x^{k}\right)$, and $h_{4}={ }^{0} h_{4}\left(x^{k}\right)$. For $\varpi=\varpi_{0}=$ const, we can take any values $w_{i}\left(x^{k}, y^{4}\right)$ because $\beta=\alpha_{i}=0$. The coefficients $n_{i}$ are found from (27) and/or, equivalently, to (41) with $\gamma=\frac{3}{2}\left|h_{3}\right|^{*}$. Integrating on $y^{4}$, this subclass of N-coefficients are computed

$$
\begin{aligned}
n_{i}= & { }_{1} n_{i}\left(x^{k}\right)+{ }_{2} n_{i}\left(x^{k}\right) \int \mathrm{d} y^{4}\left|h_{3}\right|^{-3 / 2}={ }_{1} n_{i}\left(x^{k}\right) \\
& +{ }_{2} \widetilde{n}_{i}\left(x^{k}\right)\left[c_{1}\left(x^{k}\right)+c_{2}\left(x^{k}\right) y^{4}\right]^{-2},
\end{aligned}
$$

with integration functions ${ }_{1} n_{i}\left(x^{k}\right)$ and ${ }_{2} n_{i}\left(x^{k}\right)$, or redefined ${ }_{2} \tilde{n}_{i}=-{ }_{2} n_{i} / 2 c_{2}$. The quadratic line element for this class of solutions for vacuum metrics is described by

$$
\begin{aligned}
\mathrm{d} s_{v, 4-d}^{2}= & e^{q}\left(\mathrm{~d} x^{i}\right)^{2}+\left[c_{1}\left(x^{k}\right)+c_{2}\left(x^{k}\right) y^{4}\right]^{2}\left[\mathrm{~d} t+\left({ }_{1} n_{i}\left(x^{k}\right)\right.\right. \\
& \left.\left.+{ }_{2} \tilde{n}_{i}\left(x^{k}\right)\left[c_{1}\left(x^{k}\right)+c_{2}\left(x^{k}\right) y^{4}\right]^{-2}\right) \mathrm{~d} x^{i}\right]^{2} \\
& +{ }^{0} h_{4}\left(x^{k}\right)\left[\mathrm{d} y^{4}+w_{i}\left(x^{k}, y^{4}\right) \mathrm{d} x^{i}\right]^{2} .
\end{aligned}
$$

On extra shells, this type of nonholonomic vacuum solutions are given by quadratic elements

$$
\begin{aligned}
& \mathrm{d} s_{v, 10-d 3}^{2}=e^{q}\left(\mathrm{~d} x^{i}\right)^{2}+\left\{\left[c_{1}+c_{2} y^{4}\right]^{2}[\mathrm{~d} t\right. \\
& \left.\quad+\left({ }_{1} n_{i}+{ }_{2} \tilde{n}_{i}\left[c_{1}+c_{2} y^{4}\right]^{-2}\right) \mathrm{d} x^{i}\right]^{2} \\
& \left.\quad+{ }^{0} h_{4}\left[\mathrm{~d} y^{4}+w_{i} \mathrm{~d} x^{i}\right]^{2}\right\}_{\mathrm{type} 3, s=0}+\left\{\left[{ }^{1} c_{1}+{ }^{1} c_{2} y^{6}\right]^{2}\right. \\
& \quad \times\left[\mathrm{d} y^{5}+\left({ }_{1} n_{i_{1}}+{ }_{2}{ }_{2} \widetilde{n}_{i_{1}}\left[{ }^{1} c_{1}+{ }^{1} c_{2} y^{6}\right]^{-2}\right) \mathrm{d} x^{i_{1}}\right]^{2} \\
& \left.\quad+{ }^{0} h_{6}\left[\mathrm{~d} y^{6}+{ }^{1} w_{i_{1}} \mathrm{~d} x^{i_{1}}\right]^{2}\right\}_{\mathrm{type} 3, s=1} \\
& \quad+\left\{[ { } ^ { 2 } c _ { 1 } + { } ^ { 2 } c _ { 2 } y ^ { 8 } ] ^ { 2 } \left[\mathrm{d} y^{7}+\left({ }_{1}^{2} n_{i_{2}}+{ }_{2} \widetilde{n}_{i_{2}}\left[{ }^{2} c_{1}\right.\right.\right.\right. \\
& \left.\left.\left.\left.\quad+{ }^{2} c_{2} y^{8}\right]^{-2}\right) \mathrm{~d} x^{i_{2}}\right]^{2}+{ }^{0} h_{8}\left[\mathrm{~d} y^{8}+{ }^{2} w_{i_{2}} \mathrm{~d} x^{i_{2}}\right]^{2}\right\}_{\mathrm{type}, s=2} \\
& \quad+\left\{[ { } ^ { 3 } c _ { 1 } + { } ^ { 3 } c _ { 2 } y ^ { 1 0 } ] ^ { 2 } \left[\mathrm{d} y^{9}+\left({ }_{1}^{3} n_{i_{3}}+{ }_{2}{ }_{2} \widetilde{n}_{i_{3}}\left[{ }^{2} c_{1}\right.\right.\right.\right. \\
& \left.\left.\left.\left.\quad+{ }^{2} c_{2} y^{10}\right]^{-2}\right) \mathrm{~d} x^{i_{3}}\right]^{2}+{ }^{0} h_{10}\left[\mathrm{~d} y^{10}+{ }^{3} w_{i_{3}} \mathrm{~d} x^{i_{3}}\right]^{2}\right\}_{\text {type } 3, s=3} .
\end{aligned}
$$

The generating functions are

$$
\begin{aligned}
& q\left(x^{k}\right), w_{i}\left(x^{k}, y^{4}\right), \quad{ }^{1} w_{i_{1}}\left(x^{k}, y^{4}, y^{6}\right), \\
& \quad{ }^{2} w_{i_{2}}\left(x^{k}, y^{4}, y^{a_{1}}, y^{8}\right), \quad{ }^{3} w_{i_{3}}\left(x^{k}, y^{4}, y^{a_{1}}, y^{a_{2}}, y^{10}\right)
\end{aligned}
$$

and the integration functions are

$$
\begin{aligned}
& c_{1}\left(x^{k}\right), c_{2}\left(x^{k}\right),{ }_{1} n_{i}\left(x^{k}\right),{ }_{2} \widetilde{n}_{i}\left(x^{k}\right),{ }^{0} h_{4}\left(x^{k}\right) ; \\
& { }^{1} c_{1}\left(x^{k}, y^{4}\right),{ }^{1} c_{2}\left(x^{k}, y^{4}\right),{ }_{1}^{1} n_{i_{1}}\left(x^{k}, y^{4}\right), \\
& \quad{ }_{2}^{1} \widetilde{n}_{i_{1}}\left(x^{k}, y^{4}\right),{ }^{0} h_{6}\left(x^{k}, y^{4}\right) ; \\
& { }^{2} c_{1}\left(x^{k}, y^{4}, y^{a_{1}}\right),{ }^{2} c_{2}\left(x^{k}, y^{4}, y^{a_{1}}\right),{ }_{1}^{2} n_{i_{2}}\left(x^{k}, y^{4}, y^{a_{1}}\right), \\
& { }_{2}^{2} \widetilde{n}_{i_{2}}\left(x^{k}, y^{4}, y^{a_{1}}\right),{ }^{0} h_{8}\left(x^{k}, y^{4}, y^{a_{1}}\right) ; \\
& { }^{3} c_{1}\left(x^{k}, y^{4}, y^{a_{1}}, y^{a_{2}}\right),{ }^{3} c_{2}\left(x^{k}, y^{4}, y^{a_{1}}, y^{a_{2}}\right), \\
& { }_{1}^{3} n_{i_{2}}\left(x^{k}, y^{4}, y^{a_{1}}, y^{a_{2}}\right),{ }_{2}^{3} \widetilde{n}_{i_{2}}\left(x^{k}, y^{4}, y^{a_{1}}, y^{a_{2}}\right), \\
& { }^{0} h_{10}\left(x^{k}, y^{4}, y^{a_{1}}, y^{a_{2}}\right) .
\end{aligned}
$$

Finally, we note that we can construct generic off-diagonal vacuum solutions in heterotic supergravity with different types on different shells

$$
\begin{aligned}
& \mathrm{d} s_{\text {vaccum }}^{2}=e^{q}+\{\ldots\}_{\text {type }, s=0}+\{\ldots\}_{\text {type }, s=1} \\
& +\{\ldots\}_{\text {type }, s=2}+\{\ldots\}_{\text {type }, s=3}
\end{aligned}
$$

In certain cases, the type may be the same on two or three shells, but the parametrizations of mixed types of nonholonomic vacuum solutions is given by different dependencies on shell coordinates of the generating and integration functions.

Extra-dimensional non-vacuum solutions The solutions for extra dimensions can be constructed in certain forms which are similar to the 4-d ones with (58) using new classes of generating and integration functions with dependencies on extra dimensional coordinates. For instance, we can generate solutions of the system (43)-(45) with coefficients (48) on the shell $s=1$ following a formal analogy when $\partial_{4} \rightarrow \partial_{6}$, i.e. 
$* \rightarrow *_{1}, \varpi\left(x^{k}, y^{4}\right) \rightarrow{ }^{1} \varpi\left(x^{k_{1}}, y^{6}\right)$, with ${ }^{1} \Phi\left(x^{k_{1}}, y^{4}\right)=$ $e^{1 \varpi} ; \Upsilon\left(x^{k}, y^{4}\right) \rightarrow{ }_{1} \Upsilon\left(x^{k_{1}}, y^{6}\right) \cdots$ and applying the same procedure as for the 4 -d case but extending the number of shell coordinates, respectively, for generating and integration functions.

The solutions on $s=1$ are also generated as stationary d-metrics when the coefficients do not depend on timelike coordinate $y^{3}=t$ and do not depend on the coordinate $y^{5}$. For nontrivial ${ }_{1} \Upsilon$, we have to choose ${ }^{1} \Phi^{* 1} \neq 0$. The effective gravitational field equations (43)-(45) and respective equation for ${ }^{1} \Phi$ from (48) transform (respectively) into

${ }^{1} \Phi^{*_{1}} h_{5}^{*_{1}}=2 h_{5} h_{6}{ }_{1} \Upsilon{ }^{1} \Phi$,

$\sqrt{\left|h_{5} h_{6}\right|}{ }^{1} \Phi=h_{5}^{* 1}$,

${ }^{1} n_{i_{1}}^{*_{1} *_{1}}+\left(\ln \left|h_{5}\right|^{3 / 2} /\left|h_{6}\right|\right)^{*_{1}}{ }^{1} n_{i_{1}}^{*_{1}}=0$,

${ }^{1} \Phi{ }^{1} w_{i_{1}}^{*}-\partial_{i_{1}}{ }^{1} \Phi=0$.

Prescribing ${ }_{1} \Upsilon$ and ${ }^{1} \Phi$, such equations for 6-d gravity can be integrated as in the case of $s=0$ with two vertical coordinates in 4-d gravity, i.e. integrating equations "step by step". Introducing the function $\left({ }^{1} \rho\right)^{2}:=h_{5} h_{6}$, (for extra shells the sign is different because we work with space-like coordinates) we express the first two equations above as

$$
{ }^{1} \Phi^{*_{1}} h_{5}^{*_{1}}=2\left({ }^{1} \rho\right){ }^{2}{ }_{1} \Upsilon{ }^{1} \Phi \text { and } h_{5}^{*_{1}}={ }^{1} \rho{ }^{1} \Phi \text {. }
$$

Using $h_{5}^{* 1}$ from the second equation in the first equations, we get ${ }^{1} \rho=\frac{1}{2} \frac{{ }^{1} \Phi^{* 1}}{1 \Upsilon}$. Substituting this value together with the second equation of (64) and integrating on $y^{6}$, it is possible to compute

$$
h_{5}=h_{5}^{[0]}\left(x^{k}, y^{4}\right)+\frac{1}{4} \int \mathrm{d} y^{6} \frac{\left({ }^{1} \Phi^{2}\right)^{*_{1}}}{{ }_{1} \Upsilon},
$$

where $h_{5}^{[0]}\left(x^{k}, y^{4}\right)$ is an integration function. In result, we find from the above formula $\left({ }^{1} \rho\right)^{2}$ the coefficient

$$
\begin{aligned}
h_{6} & =\frac{1}{4 h_{5}}\left(\frac{\Phi^{* 1}}{{ }_{1} \Upsilon}\right)^{2} \\
& =\frac{1}{4}\left(\frac{\Phi^{* 1}}{{ }_{1} \Upsilon}\right)^{2}\left(h_{5}^{[0]}+\frac{1}{4} \int \mathrm{d} y^{6} \frac{\left({ }^{1} \Phi^{2}\right)^{* 1}}{1 \Upsilon}\right)^{-1} .
\end{aligned}
$$

The first part of the $\mathrm{N}$-connection coefficients are found by integrating two times on $y^{6}$ in (44) written in the form

$$
{ }^{1} n_{i_{1}}^{*_{1} *_{1}}=\left(n_{i_{1}}^{* 1}\right)^{*_{1}}=-n_{i_{1}}^{*_{1}}\left(\ln \left|h_{5}\right|^{3 / 2} /\left|h_{6}\right|\right)^{*_{1}}
$$

for the coefficient ${ }^{1} \gamma$ defined in (48). This formula can be integrated two times on $y^{6}$ for explicit values of $h_{a_{1}}$ which results in

$$
\begin{aligned}
{ }^{1} n_{k_{1}}\left(x^{i}, y^{4}, y^{6}\right) & ={ }_{1}^{1} n_{k_{1}}+{ }_{2}^{1} n_{k_{1}} \int \mathrm{d} y^{6} \frac{h_{6}}{\left|h_{5}\right|^{3 / 2}} \\
& ={ }_{1}^{1} n_{k_{1}}+{ }_{2}^{1} \widetilde{n}_{k_{1}} \int \mathrm{d} y^{6} \frac{\left({ }^{1} \Phi^{* 1}\right)^{2}}{\left|h_{5}\right|^{5 / 2}\left({ }_{1} \Upsilon\right)^{2}}
\end{aligned}
$$

$$
\begin{aligned}
= & { }_{1}^{1} n_{k_{1}}+{ }_{2} \tilde{n}_{k_{1}} \int \mathrm{d} y \frac{6}{\left.{ }^{6}{ }^{1} \Phi^{* 1}\right)^{2}} \\
& \times\left|h_{5}^{[0]}+\frac{1}{4} \int \mathrm{d} y^{2} \frac{\left({ }^{1} \Phi^{2}\right)^{* 1}}{{ }_{1} \Upsilon}\right|^{-5 / 2},
\end{aligned}
$$

also containing a second set of integration functions ${ }_{1}^{1} n_{k_{1}}$ $\left(x^{i}, y^{4}\right)$ and redefined second integration function ${ }_{2}^{1} \widetilde{n}_{k_{1}}$ $\left(x^{i}, y^{4}\right)$. The second set of $\mathrm{N}$-connection coefficients on $s=1$ can be found from the linear algebraic equations (45) and express ${ }^{1} w_{i_{1}}=\partial_{i_{1}}{ }^{1} \Phi /{ }^{1} \Phi^{* 1}$.

Summarizing the above formulas for the d-connection and $\mathrm{N}$-connection coefficients on $s=1$, we write the data for generating such generic off-diagonal metrics as stationary solutions for the system (43)-(45),

$$
\begin{aligned}
h_{5}= & h_{5}^{[0]}+\frac{1}{4} \int \mathrm{d} y^{6} \frac{\left({ }^{1} \Phi^{2}\right)^{* 1}}{1 \Upsilon} ; \\
h_{6}= & \frac{1}{4}\left(\frac{\Phi^{* 1}}{1 \Upsilon}\right)^{2}\left(h_{5}^{[0]}+\frac{1}{4} \int \mathrm{d} y^{6} \frac{\left({ }^{1} \Phi^{2}\right)^{* 1}}{1 \Upsilon}\right)^{-1} ; \\
{ }^{1} n_{k_{1}}= & { }_{1}^{1} n_{k_{1}}+{ }_{2}^{1} \widetilde{n}_{k_{1}} \int \mathrm{d} y^{6} \frac{\left({ }^{1} \Phi^{* 1}\right)^{2}}{(1 \Upsilon)^{2}} \\
& \times\left|h_{5}^{[0]}+\frac{1}{4} \int \mathrm{d} y^{6} \frac{\left({ }^{1} \Phi^{2}\right)^{* 1}}{1 \Upsilon}\right|^{-5 / 2} ; \\
{ }^{1} w_{i_{1}}= & \partial_{i_{1}}{ }^{1} \Phi /{ }^{1} \Phi^{* 1} .
\end{aligned}
$$

The quadratic elements for off-diagonal 6-d stationary configurations in heterotic supergravity with nonholonomically induced torsion are constructed using above $\mathrm{N}$-adapted coefficients

$$
\begin{aligned}
\mathrm{d} s_{K 6 d}^{2}= & g_{\alpha_{1} \beta_{1}}\left(x^{k}, y^{4}, y^{a_{1}}\right) \mathrm{d} u^{\alpha_{1}} \mathrm{~d} u^{\beta_{1}}=\mathrm{d} s_{K 4 d}^{2}[\text { see (59)] } \\
& +\left[h_{5}^{[0]}+\frac{1}{4} \int \mathrm{d} y^{6} \frac{\left({ }^{1} \Phi^{2}\right)^{* 1}}{{ }_{1} \Upsilon}\right] \\
& \times\left[\mathrm{d} y^{5}+\left({ }_{1} n_{k_{1}}+{ }_{2} \tilde{n}_{k_{1}} \int \mathrm{d} y^{6} \frac{\left({ }^{1} \Phi^{* 1}\right)^{2}}{\left({ }_{1} \Upsilon\right)^{2}}\right.\right. \\
& \left.\times\left\lfloor h_{5}^{[0]}+\left.\frac{1}{4} \int \mathrm{d} y^{6} \frac{\left({ }^{1} \Phi^{2}\right)^{* 1}}{1 \Upsilon}\right|^{-5 / 2}\right) \mathrm{d} x^{k_{1}}\right] \\
& +\frac{1}{4}\left(\frac{{ }^{1} \Phi^{* 1}}{{ }_{1} \Upsilon}\right)^{2}\left(h_{5}^{[0]}+\frac{1}{4} \int \mathrm{d} y^{6} \frac{\left({ }^{1} \Phi^{2}\right)^{* 1}}{{ }_{1} \Upsilon}\right)^{-1} \\
& \times\left[\mathrm{d} y^{6}+\frac{\partial_{i_{1}}{ }^{1} \Phi}{1 \Phi^{* 1}}\right] .
\end{aligned}
$$

We can repeat the method of constructing solutions for $s=1$ to next shells $s=2$ and $s=3$ and integrate the systems (46) and (47), respectively. It is necessary to extend the formulas for generating and integration functions and effective sources recurrently as we provided above for redefinition of respective values for $s=0$ to $s=1$. The quadratic elements are parametrized in corresponding forms: 


$$
\begin{aligned}
\mathrm{d} s_{K 8 d}^{2}= & g_{\alpha_{2} \beta_{2}}\left(x^{k}, y^{4}, y^{a_{1}}, y^{a_{2}}\right) \mathrm{d} u^{\alpha_{2}} \mathrm{~d} u^{\beta_{2}} \\
= & \mathrm{d} s_{K 6 d}^{2}[\operatorname{see}(65)]+\left[h_{6}^{[0]}+\frac{1}{4} \int \mathrm{d} y^{8} \frac{\left({ }^{2} \Phi^{2}\right)^{*_{2}}}{2 \Upsilon}\right] \\
& \times\left[\mathrm{d} y^{7}+\left({ }_{1}^{2} n_{k_{2}}+{ }_{2} \widetilde{n}_{k_{2}} \int \mathrm{d} y^{8} \frac{\left({ }^{2} \Phi^{* 2}\right)^{2}}{\left({ }_{2} \Upsilon\right)^{2}}\right.\right. \\
& \left.\times\left\lfloor h_{7}^{[0]}+\left.\frac{1}{4} \int \mathrm{d} y^{8} \frac{\left({ }^{2} \Phi^{2}\right)^{*_{2}}}{2 \Upsilon}\right|^{-5 / 2}\right) \mathrm{d} x^{k_{2}}\right] \\
& +\frac{1}{4}\left(\frac{{ }^{2} \Phi^{* 2}}{{ }_{2} \Upsilon}\right)^{2}\left(h_{7}^{[0]}+\frac{1}{4} \int \mathrm{d} y^{8} \frac{\left({ }^{2} \Phi^{2}\right)^{*_{2}}}{2 \Upsilon}\right)^{-1} \\
& \times\left[\mathrm{d} y^{8}+\frac{\partial_{i_{2}}{ }^{2} \Phi}{2} \Phi^{* 2}\right]
\end{aligned}
$$

and

$$
\begin{aligned}
\mathrm{d} s_{K 10 d}^{2}= & g_{\alpha_{2} \beta_{2}}\left(x^{k}, y^{4}, y^{a_{1}}, y^{a_{2}}, y^{a_{2}}\right) \mathrm{d} u^{\alpha_{3}} \mathrm{~d} u^{\beta_{3}} \\
= & \mathrm{d} s_{K 8 d}^{2}[\operatorname{see}(66)]+\left[h_{8}^{[0]}+\frac{1}{4} \int \mathrm{d} y^{10} \frac{\left({ }^{3} \Phi^{2}\right)^{* 3}}{3 \Upsilon}\right] \\
& \times\left[\mathrm{d} y^{9}+\left({ }_{1}^{3} n_{k_{3}}+{ }_{2}^{3} \widetilde{n}_{k_{3}} \int \mathrm{d} y^{10} \frac{\left({ }^{3} \Phi^{* 3}\right)^{2}}{\left({ }_{3} \Upsilon\right)^{2}}\right.\right. \\
& \left.\left.\times\left|h_{9}^{[0]}+\frac{1}{4} \int \mathrm{d} y^{10} \frac{\left({ }^{3} \Phi^{2}\right)^{* 3}}{3 \Upsilon}\right|^{-5 / 2}\right) \mathrm{~d} x^{k_{3}}\right] \\
& +\frac{1}{4}\left(\frac{{ }^{3} \Phi^{* 3}}{{ }_{3} \Upsilon}\right)^{2}\left(h_{9}^{[0]}+\frac{1}{4} \int \mathrm{d} y^{10} \frac{\left({ }^{3} \Phi^{2}\right)^{*_{3}}}{3 \Upsilon}\right)^{-1} \\
& \times\left[\mathrm{d} y^{10}+\frac{\partial_{i_{3}}{ }^{3} \Phi}{{ }^{3} \Phi^{* 3}}\right] .
\end{aligned}
$$

The generic off-diagonal d-metrics (67) define exact stationary solutions with Killing symmetries on $\partial_{t}$ and $\partial_{9}$ in effective nonholonomic 10-d gravity with sources (34) being determined by the equations of motion in heterotic supergravity (12).

\subsubsection{A nonlinear symmetry of generating functions and effective sources}

There is an important nonlinear symmetry relating nontrivial generating functions and effective sources considered in the above classes of solutions, used in differen forms in [42,43]. It allows us to redefine the generating functions and introduce effective cosmological constants instead of effective sources. Let us study these properties in the case of heterotic string gravity.

Changing the generating data $(\Phi, \Upsilon) \leftrightarrow(\tilde{\Phi}, \widetilde{\Lambda}=$ const $)$, where

$$
\begin{aligned}
\frac{\left(\Phi^{2}\right)^{*}}{\Upsilon} & =\frac{\left(\widetilde{\Phi}^{2}\right)^{*}}{\widetilde{\Lambda}}, \text { which is equivalent to } \\
\widetilde{\Phi}^{2} & =\widetilde{\Lambda} \int \mathrm{d} y^{4} \Upsilon^{-1}\left(\Phi^{2}\right)^{*} \text { and } / \text { or } \\
\Phi^{2} & =\widetilde{\Lambda}^{-1} \int \mathrm{d} y^{4} \Upsilon\left(\widetilde{\Phi}^{2}\right)^{*}
\end{aligned}
$$

up to certain classes of integration functions depending on coordinates $x^{i}$, we express the solutions (56) and (57) of the system of nonlinear PDEs (49) and (51) in two equivalent forms,

$$
\begin{aligned}
h_{3} & =h_{3}[\Phi]=h_{3}^{[0]}\left(x^{k}\right)-\frac{1}{4} \int \mathrm{d} y^{4} \frac{\left(\Phi^{2}\right)^{*}}{\Upsilon} \\
& =h_{3}[\widetilde{\Phi}]=h_{3}^{[0]}\left(x^{k}\right)-\frac{\widetilde{\Phi}^{2}}{4 \widetilde{\Lambda}},
\end{aligned}
$$

and

$$
\begin{aligned}
h_{4} & =h_{4}[\Phi]=-\frac{1}{4 h_{3}[\Phi]}\left(\frac{\Phi^{*}}{\Upsilon}\right)^{2}=h_{4}[\widetilde{\Phi}] \\
& =-\frac{1}{4 h_{3}[\widetilde{\Phi}]} \frac{\left(\widetilde{\Phi}^{*}\right)^{2}}{\widetilde{\Lambda}^{2}} .
\end{aligned}
$$

Having defined two equivalent nonlinear formulas for $h_{a}[\Phi]$ $=h_{a}[\widetilde{\Phi}]$, we can express in two equivalent forms the Nadapted coefficients for the d-metric and $\mathrm{N}$-connection (58). In the second case, the $\mathrm{N}$-connection coefficients are computed using integrals on $\mathrm{d} y^{4}$ for certain values determined by $(\tilde{\Phi}, \widetilde{\Lambda})$ via (68) and integration functions,

$n_{k}={ }_{1} n_{k}+{ }_{2} n_{k} \int \mathrm{d} y^{4} \frac{h_{4}[\widetilde{\Phi}]}{\left|h_{3}[\widetilde{\Phi}]\right|^{3 / 2}}$ and

$w_{i}=\frac{\partial_{i} \Phi}{\Phi^{*}}=\frac{\partial_{i} \Phi^{2}}{\left(\Phi^{2}\right)^{*}}=\frac{\partial_{i} \int \mathrm{d} y^{4} \Upsilon\left(\widetilde{\Phi}^{2}\right)^{*}}{\Upsilon\left(\widetilde{\Phi}^{2}\right)^{*}}$.

We observe that if the effective source $\Upsilon=\Upsilon\left(x^{k}\right)$ does not depend on $y^{4}$, we have the same expression for $w_{i}$ in terms of generating functions, $w_{i}=\frac{\partial_{i} \Phi}{\Phi^{*}}=\frac{\partial_{i} \widetilde{\Phi}}{\widetilde{\Phi}^{*}}$.

The nonlinear symmetry reflects the property of changing the effective sources mentioned in (34),

$\Upsilon\left(x^{k}, y^{4}\right) \rightarrow \Lambda={ }^{H} \Lambda+{ }^{F} \Lambda+{ }^{R} \Lambda$, for ${ }^{\phi} \Lambda=0$.

We can identify $\tilde{\Lambda}$ with $\Lambda$, or any other value ${ }^{H} \Lambda,{ }^{F} \Lambda,{ }^{R} \Lambda$, and their sums, depending on the class of models with effective gauge interactions we consider in our work.

In a similar form, using recurrent formulas, we can prove the existence of such nonlinear symmetries generalizing (68),

$$
\begin{aligned}
& s=0: \frac{\left(\Phi^{2}\right)^{*}}{\Upsilon}=\frac{\left(\widetilde{\Phi}^{2}\right)^{*}}{\widetilde{\Lambda}} \text {, i.e. } \widetilde{\Phi}^{2}=\widetilde{\Lambda} \int \mathrm{d} y^{4} \Upsilon^{-1}\left(\Phi^{2}\right)^{*} \\
& \text { and/or } \Phi^{2}=\widetilde{\Lambda}^{-1} \int \mathrm{d} y^{4} \Upsilon\left(\widetilde{\Phi}^{2}\right)^{*} ; \\
& s=1: \frac{\left({ }^{1} \Phi^{2}\right)^{* 1}}{{ }_{1} \Upsilon}=\frac{\left({ }^{1} \widetilde{\Phi}^{2}\right)^{* 1}}{{ }_{1} \widetilde{\Lambda}}, \\
& \text { i.e. }{ }^{1} \widetilde{\Phi}^{2}={ }_{1} \widetilde{\Lambda} \int \mathrm{d} y^{6}\left({ }_{1} \Upsilon\right)^{-1}\left({ }^{1} \Phi^{2}\right)^{{ }_{1}} \\
& \text { and/or }\left({ }^{1} \Phi\right)^{2}={ }_{1} \widetilde{\Lambda}^{-1} \int \mathrm{d} y^{6}{ }_{1} \Upsilon\left({ }^{1} \widetilde{\Phi}^{2}\right)^{* 1} ; \\
& s=2: \frac{\left({ }^{2} \Phi^{2}\right)^{* 2}}{{ }_{2} \Upsilon}=\frac{\left({ }^{2} \widetilde{\Phi}^{2}\right)^{* 2}}{{ }_{2} \widetilde{\Lambda}},
\end{aligned}
$$




$$
\begin{aligned}
& \text { i.e. }{ }^{2} \widetilde{\Phi}^{2}={ }_{2} \widetilde{\Lambda} \int \mathrm{d} y^{8}\left({ }_{2} \Upsilon\right)^{-1}\left({ }^{2} \Phi^{2}\right)^{*_{2}} \\
& \text { and/or }\left({ }^{2} \Phi\right)^{2}={ }_{2} \widetilde{\Lambda}^{-1} \int \mathrm{d} y^{8}{ }_{2} \Upsilon\left({ }^{2} \widetilde{\Phi}^{2}\right)^{*_{2}} ; \\
& s=3: \frac{\left({ }^{3} \Phi^{2}\right)^{* 3}}{{ }_{3} \Upsilon}=\frac{\left({ }^{3} \widetilde{\Phi}^{2}\right)^{* 3}}{{ }_{3} \widetilde{\Lambda}} \\
& \text { i.e. }{ }^{3} \widetilde{\Phi}^{2}={ }_{3} \widetilde{\Lambda} \int \mathrm{d} y^{10}\left({ }_{3} \Upsilon\right)^{-1}\left({ }^{3} \Phi^{2}\right)^{* 3} \\
& \text { and/or }\left({ }^{3} \Phi\right)^{2}={ }_{3} \widetilde{\Lambda}^{-1} \int \mathrm{d} y^{10}{ }_{3} \Upsilon\left({ }^{3} \widetilde{\Phi}^{2}\right)^{* 3}
\end{aligned}
$$

We consider the convention that $\widehat{\Upsilon}_{9}^{9}=\widehat{\Upsilon}_{10}^{10}={ }_{3} \Upsilon\left(x^{k}, y^{a}\right.$, $\left.y^{a_{1}}, y^{a_{2}}, y^{10}\right) \rightarrow{ }_{3} \Lambda={ }_{3}^{\phi} \Lambda+{ }_{3}^{H} \Lambda+{ }_{3}^{F} \Lambda+{ }_{3}^{R} \Lambda$ and identify ${ }_{3} \widetilde{\Lambda}$ with ${ }_{3} \Lambda$. Such nonlinear transforms can be used for simplifications of formulas for generic off-diagonal solutions. Prescribing certain effective matter field initial distributions, we can redefine the constructions equivalently, via new classes of generating functions, for effective cosmological constants.

Finally, we note that Eq. (67) simplifies for $\left({ }^{3} \Phi,{ }_{3} \Upsilon\right) \rightarrow$ $\left({ }^{3} \widetilde{\Phi},{ }_{3} \widetilde{\Lambda}\right)$ if ${ }_{3} \Upsilon={ }_{3} \Upsilon\left(x^{i}, y^{4}, y^{a_{1}}, y^{a_{2}}\right)$,

$$
\begin{aligned}
\mathrm{d} s_{K 10 d}^{2}= & g_{\alpha_{2} \beta_{2}}\left(x^{k}, y^{4}, y^{a_{1}}, y^{a_{2}}, y^{a_{2}},{ }^{3} \widetilde{\Phi},{ }_{3} \widetilde{\Lambda}\right) \mathrm{d} u^{\alpha_{3}} \mathrm{~d} u^{\beta_{3}} \\
= & \mathrm{d} s_{K 8 d}^{2}[\text { see }(66)]+\left[h_{8}^{[0]}+\frac{\left({ }^{3} \widetilde{\Phi}^{2}\right)}{4{ }_{3} \widetilde{\Lambda}}\right] \\
& \times\left[\mathrm{d} y^{9}+\left({ }_{1}^{3} n_{k_{3}}+{ }_{2} \widetilde{n}_{k_{3}} \int \mathrm{d} y^{10}\left({ }^{3} \widetilde{\Phi}^{* 3}\right)^{2}\right.\right. \\
& \left.\left.\times\left|h_{9}^{[0]}+\frac{\left({ }^{3} \widetilde{\Phi}^{2}\right)}{4{ }_{3} \widetilde{\Lambda}}\right|^{-5 / 2}\right) \mathrm{~d} x^{k_{3}}\right] \\
& +\frac{1}{4} \frac{\left({ }^{3} \widetilde{\Phi}^{* 3}\right)^{2}}{{ }_{3} \widetilde{\Lambda}{ }_{3} \Upsilon \mid}\left(h_{9}^{[0]}+\frac{\left({ }^{3} \widetilde{\Phi}^{2}\right)}{4{ }_{3} \widetilde{\Lambda}}\right)^{-1} \\
& \times\left[\mathrm{d} y^{10}+\frac{\partial_{i_{3}}{ }^{3} \widetilde{\Phi}}{{ }_{3} \widetilde{\Phi}^{* 3}}\right] .
\end{aligned}
$$

Such generic off-diagonal configurations are described by generic off-diagonal metrics with effective cosmological constants, generalized generating functions and integration functions. The contributions of ${ }_{3} \Upsilon\left(x^{i}, y^{4}, y^{a_{1}}, y^{a_{2}}\right)$ can be encoded into generating and integrating functions.

\subsubsection{The Levi-Civita conditions}

In general, a solution constructed for a generic off-diagonal metric (24) and canonical d-connections ${ }^{s} \widehat{\mathbf{D}}$ is characterized by nonholonomically induced d-torsion coefficients $\widehat{\mathbf{T}}_{\alpha_{s} \beta_{s}}^{\gamma_{s}}$ (7) completely defined by the $\mathrm{N}$-connection and dmetric structure. The zero torsion conditions (8) can be satisfied by a subclass of nonholonomic distributions determined by corresponding parametrizations of the generating and integration functions and sources. By straightforward computations (see the details in Refs. [35,38-40]), we can verify that if the coefficients of $\mathrm{N}$-adapted frames and ${ }^{s} v$ components of d-metrics are subject to the respective conditions

$$
\begin{aligned}
& s=0: w_{i}^{*}=\mathbf{e}_{i} \ln \sqrt{\left|h_{4}\right|}, \mathbf{e}_{i} \ln \sqrt{\left|h_{3}\right|}=0, \\
& \partial_{i} w_{j}=\partial_{j} w_{i} \text { and } n_{i}^{*}=0 ; \\
& s=1:{ }^{1} w_{i_{1}}^{*}={ }^{1}{ }_{\mathbf{e}_{i_{1}}} \ln \sqrt{\left|h_{6}\right|},{ }^{1} \mathbf{e}_{i_{1}} \ln \sqrt{\left|h_{5}\right|}=0, \\
& \partial_{i_{1}}{ }^{1} w_{j_{1}}=\partial_{j_{1}}{ }^{1} w_{i_{1}} \text { and }{ }^{1} n_{i_{1}}^{* 1}=0 ; \\
& s=2:{ }^{2} w_{i_{2}}^{* 2}={ }^{2} \mathbf{e}_{i_{2}} \ln \sqrt{\left|h_{8}\right|},{ }^{2} \mathbf{e}_{i_{2}} \ln \sqrt{\left|h_{7}\right|}=0, \\
& \partial_{i_{2}}{ }^{2} w_{j_{2}}=\partial_{j_{2}}{ }^{2} w_{i_{2}} \text { and }{ }^{2} n_{i_{2}}^{* 2}=0 ; \\
& s=3:{ }^{3} w_{i_{3}}^{* 3}={ }^{3} \mathbf{e}_{i_{3}} \ln \sqrt{\left|h_{10}\right|},{ }^{3} \mathbf{e}_{i_{3}} \ln \sqrt{\left|h_{9}\right|}=0, \\
& \partial_{i_{3}}{ }^{3} w_{j_{3}}=\partial_{j_{3}}{ }^{3} w_{i_{3}} \text { and }{ }^{3} n_{i_{3}}^{* 3}=0 ;
\end{aligned}
$$

all d-torsion coefficients are zero.

The $n$-coefficients solve the conditions (71) if

$s=0:{ }_{2} n_{k}\left(x^{i}\right)=0$ and $\partial_{i}{ }_{1} n_{j}\left(x^{k}\right)=\partial_{j}{ }_{1} n_{i}\left(x^{k}\right)$;

$s=1:{ }_{2}^{1} n_{k_{1}}\left(x^{i_{1}}\right)=0$ and $\partial_{i_{1}}{ }_{1}^{1} n_{j_{1}}\left(x^{k_{1}}\right)=\partial_{j_{1}}{ }_{1}^{1} n_{i_{1}}\left(x^{k_{1}}\right)$;

$s=2:{ }_{2}^{2} n_{k_{2}}\left(x^{i_{2}}\right)=0$ and $\partial_{i_{2}}{ }_{1}^{2} n_{j_{2}}\left(x^{k_{2}}\right)=\partial_{j_{2}}{ }_{1}^{2} n_{i_{2}}\left(x^{k_{2}}\right)$;

$s=3:{ }_{2}^{3} n_{k_{3}}\left(x^{i_{3}}\right)=0$ and $\partial_{i_{3}}{ }_{1}^{3} n_{j_{3}}\left(x^{k_{3}}\right)=\partial_{j_{3}}{ }_{1}^{3} n_{i_{3}}\left(x^{k_{3}}\right)$.

The explicit form of solutions of constraints on $w_{k}$ derived from (71) depends on the class of vacuum or non-vacuum metrics we try to construct; see for details [43]. For instance, if we choose a generating function $\Phi=\breve{\Phi}\left(x^{i}, y^{4}\right)$, for which $\left(\partial_{i} \check{\Phi}\right)^{*}=\partial_{i} \check{\Phi}^{*}$, we solve the conditions for $w_{i}$ in (71) in explicit form if $\Upsilon=$ const, or if such an effective source can be expressed as a functional, $\Upsilon\left(x^{i}, y^{4}\right)=\Upsilon[\check{\Phi}]$. The third condition for $s=0, \partial_{i} w_{j}=\partial_{j} w_{i}$, can be satisfied for any generating function $\check{A}=\check{A}\left(x^{k}, y^{4}\right)$ for which $w_{i}=\breve{w}_{i}=$ $\partial_{i} \check{\Phi} / \partial_{4} \check{\Phi}=\partial_{i} \check{A}$. Following similar considerations for shells $s=1,2,3$, we formulate the LC-conditions for generating functions,

$$
\begin{aligned}
& s=0: \quad \Phi=\breve{\Phi}\left(x^{i}, y^{4}\right),\left(\partial_{i} \check{\Phi}\right)^{*}=\partial_{i} \check{\Phi}^{*}, \\
& \check{w}_{i}=\partial_{i} \check{\Phi} / \partial_{4} \check{\Phi}=\partial_{i} \check{A} \text {; } \\
& \Upsilon\left(x^{i}, y^{4}\right)=\Upsilon[\check{\Phi}] \text {, or } \Upsilon=\text { const; } \\
& s=1:{ }^{1} \Phi={ }^{1} \breve{\Phi}\left(u^{\tau}, y^{6}\right),\left(\partial_{i_{1}}{ }^{1} \breve{\Phi}\right)^{*_{1}}=\partial_{i_{1}}{ }^{1} \check{\Phi}^{*_{1}}, \\
& \partial_{\alpha}{ }^{1} \check{\Phi} / \partial_{6}{ }^{1} \check{\Phi}=\partial_{\alpha}{ }^{1} \check{A} ;{ }_{1}^{1} n_{\tau}=\partial_{\tau}{ }^{1} n\left(u^{\beta}\right) \text {; } \\
& \Upsilon\left(x^{i}, y^{4}\right)=\Upsilon[\check{\Phi}], \quad \text { or } \Upsilon=\text { const; } \\
& s=2:{ }^{2} \Phi={ }^{2} \breve{\Phi}\left(u^{\tau_{1}}, y^{8}\right), \partial_{8} \partial_{\tau_{1}}{ }^{2} \check{\Phi}=\partial_{\tau_{1}} \partial_{8}{ }^{2} \check{\Phi} ; \\
& \partial_{\alpha_{1}}{ }^{2} \breve{\Phi} / \partial_{8}{ }^{2} \breve{\Phi}=\partial_{\alpha_{2}}{ }^{2} \breve{A} ;{ }_{1}^{2} n_{\tau_{1}}=\partial_{\tau_{1}}{ }^{2} n\left(u^{\beta_{1}}\right) \text {; } \\
& \Upsilon\left(x^{i}, y^{4}\right)=\Upsilon[\check{\Phi}], \quad \text { or } \Upsilon=\text { const; } \\
& s=3:{ }^{2} \Phi={ }^{2} \breve{\Phi}\left(u^{\tau_{1}}, y^{8}\right), \partial_{8} \partial_{\tau_{1}}{ }^{2} \check{\Phi}=\partial_{\tau_{1}} \partial_{8}{ }^{2} \check{\Phi} ; \\
& \partial_{\alpha_{1}} ;{ }^{2} \check{\Phi} / \partial_{8}{ }^{2} \check{\Phi}=\partial_{\alpha_{2}}{ }^{2} \check{A} ;{ }_{1}^{2} n_{\tau_{1}}=\partial_{\tau_{1}}{ }^{2} n\left(u^{\beta_{1}}\right) \text {; } \\
& \Upsilon\left(x^{i}, y^{4}\right)=\Upsilon[\check{\Phi}] \text {, or } \Upsilon=\text { const. }
\end{aligned}
$$

Imposing respective conditions from (72) and (73) on the coefficients of (58), we define such a class of quadratic ele- 
ments for off-diagonal 4-d stationary configurations in heterotic supergravity with zero induced torsion,

$$
\begin{aligned}
\mathrm{d} s_{K 4 d}^{2}= & \check{g}_{\alpha \beta}\left(x^{k}, y^{4}\right) \mathrm{d} u^{\alpha} \mathrm{d} u^{\beta}=e^{q}\left[\left(\mathrm{~d} x^{1}\right)^{2}+\left(\mathrm{d} x^{2}\right)^{2}\right] \\
& +\left[h_{3}^{[0]}\left(x^{k}\right)-\frac{1}{4} \int \mathrm{d} y^{4} \frac{\left(\check{\Phi}^{2}\right)^{*}}{\Upsilon[\check{\Phi}]}\right]\left[\mathrm{d} t+\left(\partial_{k} n\right) \mathrm{d} x^{k}\right]^{2} \\
& -\frac{1}{4}\left(\frac{\check{\Phi}^{*}}{\Upsilon[\check{\Phi}]}\right)^{2}\left(h_{3}^{[0]}-\frac{1}{4} \int \mathrm{d} y^{4} \frac{\left(\check{\Phi}^{2}\right)^{*}}{\Upsilon[\check{\Phi}]}\right)^{-1} \\
& \times\left[\mathrm{d} y^{4}+\left(\partial_{i} \check{A}\right) \mathrm{d} x^{i}\right]^{2} .
\end{aligned}
$$

We can impose similar conditions and generate exact offdiagonal solutions with respective data $\left({ }^{5} \check{\mathbf{g}},{ }^{s} \check{\mathbf{N}},{ }^{s} \check{\nabla}\right)$ for which all nonholonomic torsions are zero, but this will impose very strong restrictions on the dynamics of effective matter fields on extra shells in heterotic supergravity. In order to consider realistic solutions in string gravity with 6-d interior almost-K ähler configurations, the torsion is positively not zero. Such $10-\mathrm{d}$ solutions are conventionally parameterized as $\left(s=0: \check{g}, N, \nabla ; s=1,2,3:{ }^{s} \mathbf{g},{ }^{s} \mathbf{N},{ }^{s} \widehat{\mathbf{D}}\right)$, for stationary configurations with Killing symmetry on $\partial_{t}$ and $\partial_{9}$. Hereafter we shall work with solutions with nontrivial nonholonomically induced torsions considering that it is always possible to state additional constraints resulting in LC-configurations in the 4-d case or extra dimensions.

\subsection{Small $\mathrm{N}$-adapted nonholonomic stationary deformations}

We can construct very general classes of generic off-diagonal stationary solutions in heterotic supergravity. It is not clear what physical meaning these configurations may have for generating and integration functions with arbitrary data. Using small off-diagonal deformations of some known physically important solutions we can understand physical properties of such solutions characterized by locally anisotropic polarization/running of constants and nonlinear off-diagonal gravitational interactions determined by (super-) string corrections.

We consider a "prime" pseudo-Riemannian metric of type $\stackrel{\circ}{\mathbf{g}}=\left[\stackrel{\circ}{g}_{i}, \stackrel{\circ}{h}_{a_{s}}, \stackrel{\circ}{N}_{b_{s}}^{j_{s}}\right]$ when

$$
\begin{aligned}
& \mathrm{d} s^{2}=\stackrel{\circ}{g}_{i}\left(x^{k}\right)\left(\mathrm{d} x^{i}\right)^{2}+\stackrel{\circ}{h}_{a}\left(x^{k}, y^{4}\right)\left(\mathrm{d} y^{a}\right)^{2}\left(\stackrel{\mathfrak{e}}{a}^{a}\right)^{2} \\
& +\stackrel{\circ}{g}_{a_{1}}\left(x^{k}, y^{4}, y^{6}\right)\left({\stackrel{\circ}{a_{1}}}^{2}\right)^{2} \\
& +\stackrel{\circ}{g}_{a_{2}}\left(x^{k}, y^{4}, y^{a_{1}}, y^{8}\right)\left({\stackrel{\circ}{a^{2}}}^{2}\right)^{2} \\
& +\stackrel{\circ}{g}_{a_{3}}\left(x^{k}, y^{4}, y^{a_{1}}, y^{a_{2}}, y^{10}\right)\left(\stackrel{\bullet}{\mathbf{e}}^{a_{3}}\right)^{2},
\end{aligned}
$$

where ${\stackrel{\mathrm{e}}{a_{s}}}$ are $\mathrm{N}$-elongated differentials. Such a metric is diagonalizable if there is a coordinate transform $u^{\alpha_{s}^{\prime}}=$ $u^{\alpha_{s}^{\prime}}\left(u^{\alpha_{s}}\right)$ when $\mathrm{d} s^{2}=\stackrel{\circ}{g}_{i^{\prime}}\left(x^{k \prime}\right)\left(\mathrm{d} x^{i^{\prime}}\right)^{2}+\stackrel{\circ}{h}_{a_{s}^{\prime}}\left(x^{k \prime}\right)\left(\mathrm{d} y^{a_{s}^{\prime}}\right)^{2}$, with ${ }^{s}{\stackrel{\circ}{i_{s}}}_{i_{s}}={ }^{s}{\stackrel{\circ}{i_{s}}}=0$. To construct exact solutions with non-singular coordinates it is important to work with "for- mal" off-diagonal parameterizations when the coefficients ${ }^{s} \stackrel{\circ}{w}_{i_{s}}$ and/or ${ }^{s}{\stackrel{\circ}{n_{s}}}_{s_{s}}$ are not zero but the anholonomy coefficients $\stackrel{\circ}{W}_{\beta_{s} \gamma_{s}}^{\alpha_{s}}\left(u^{\mu_{s}}\right)=0$; see (4). We suppose that some data $\left(\stackrel{\circ}{g}_{i^{\prime}},{\stackrel{\circ}{a_{s}^{\prime}}}\right)$ may define a known physically important diagonal exact solution in GR or heterotic string gravity (for instance, a black hole, BH, configuration of Kerr or Schwarzschild type). Our goal is to study certain small generic off-diagonal parametric deformations of the prime d-metric and $\mathrm{N}$-connection coefficients (75) into certain target metrics

$$
\begin{aligned}
& \mathrm{d} s^{2}=\eta_{i} \stackrel{\circ}{g}_{i}\left(\mathrm{~d} x^{i}\right)^{2}+\eta_{a_{s}} \stackrel{\circ}{a}_{a_{s}}\left(\mathbf{e}^{a_{s}}\right)^{2}, \\
& \mathbf{e}^{3}=\mathrm{d} t+{ }^{n} \eta_{i} \stackrel{\circ}{n}_{i} \mathrm{~d} x^{i}, \quad \mathbf{e}^{4}=\mathrm{d} y^{4}+{ }^{w} \eta_{i} \stackrel{\leftrightarrow}{w}_{i} \mathrm{~d} x^{i}, \\
& \mathbf{e}^{5}=\mathrm{d} y^{5}+{ }^{n} \eta_{i_{1}} \stackrel{\circ}{n}_{i_{1}} \mathrm{~d} x^{i_{1}}, \quad \mathbf{e}^{6}=\mathrm{d} y^{6}+{ }^{w} \eta_{i_{1}}{\stackrel{\circ}{w_{1}}}_{1} \mathrm{~d} x^{i_{1}}, \\
& \mathbf{e}^{7}=\mathrm{d} y^{7}+{ }^{n} \eta_{i_{2}}{\stackrel{\circ}{i_{2}}}_{i_{2}} \mathrm{~d} x^{i_{2}}, \quad \mathbf{e}^{8}=\mathrm{d} y^{8}+{ }^{w} \eta_{i_{2}}{\stackrel{\circ}{i_{s}}}_{i_{s}} \mathrm{~d} x^{i_{s}}, \\
& \mathbf{e}^{9}=\mathrm{d} y^{9}+{ }^{n} \eta_{i_{3}}{\stackrel{\circ}{i_{3}}}_{i_{3}} \mathrm{~d} x^{i_{3}}, \quad \mathbf{e}^{10}=\mathrm{d} y^{10}+{ }^{w} \eta_{i_{3}}{\stackrel{\circ}{i_{3}}}_{i_{3}} \mathrm{~d} x^{i_{3}},
\end{aligned}
$$

where the coefficients $\left(g_{\alpha_{s}}=\eta_{\alpha_{s}} \stackrel{\circ}{\alpha_{s}},{ }^{w} \eta_{i_{s}}{\stackrel{\circ}{i_{s}}},{ }^{n} \eta_{i_{s}} n_{i_{s}}\right)$ define, for instance, a d-metric ${ }^{s} \mathbf{g}$ (24) as a solution of nonholonomic equations of motion in heterotic string gravity (12) rewritten as a nonlinear system of PDEs in nonholonomic 10-d gravity (39)-(47).

Let us construct exact solutions, for instance, of type (67). For certain well-defined conditions, we can show an expression using d-metric and $\mathrm{N}$-connection deformations stated in explicit form on all shells for the d-metric and $n$ - and $w$ coefficients:

For the coefficients of d-metrics,

$$
\begin{aligned}
& \eta_{i}=1+\varepsilon \chi_{i}\left(x^{k}\right), \quad \eta_{a}=1+\varepsilon \chi_{a}\left(x^{k}, y^{4}\right), \\
& \eta_{a_{1}}=1+\varepsilon \chi_{a_{1}}\left(x^{k}, y^{4}, y^{6}\right), \\
& \eta_{a_{2}}=1+\varepsilon \chi_{a_{2}}\left(x^{k}, y^{4}, y^{a_{1}}, y^{8}\right), \\
& \eta_{a_{3}}=1+\varepsilon \chi_{a_{3}}\left(x^{k}, y^{4}, y^{a_{1}}, y^{a_{2}}, y^{10}\right)
\end{aligned}
$$

and for the coefficients of $\mathrm{N}$-connection,

$$
\begin{aligned}
& { }^{n} \eta_{i}=1+\varepsilon{ }^{n} \eta_{i}\left(x^{k}, y^{4}\right), \quad{ }^{w} \eta_{i}=1+\varepsilon^{w} \chi_{i}\left(x^{k}, y^{4}\right), \\
& { }^{n} \eta_{i_{1}}=1+\varepsilon{ }^{n} \eta_{i_{1}}\left(x^{k}, y^{4}, y^{6}\right), \\
& { }^{w} \eta_{i_{1}}=1+\varepsilon{ }^{w} \chi_{i_{1}}\left(x^{k}, y^{4}, y^{6}\right), \\
& { }^{n} \eta_{i_{2}}=1+\varepsilon{ }^{n} \eta_{i_{2}}\left(x^{k}, y^{4}, y^{a_{1}}, y^{8}\right), \\
& { }^{w} \eta_{i_{2}}=1+\varepsilon{ }^{w} \chi_{i_{2}}\left(x^{k}, y^{4}, y^{a_{1}}, y^{8}\right), \\
& { }^{n} \eta_{i_{3}}=1+\varepsilon{ }^{n} \eta_{i_{3}}\left(x^{k}, y^{4}, y^{a_{1}}, y^{a_{2}}, y^{10}\right), \\
& { }^{w} \eta_{i_{3}}=1+\varepsilon^{w} \chi_{i_{3}}\left(x^{k}, y^{4}, y^{a_{1}}, y^{a_{2}}, y^{10}\right),
\end{aligned}
$$

for a small parameter $0 \leq \varepsilon \ll 1$, when (76) transforms into (75) for $\varepsilon \rightarrow 0$ (which in turn, can be diagonalized). In general, there are no smooth limits from such nonholonomic deformations which can be satisfied for arbitrary generation and integration functions, integration constants, and general (effective) sources on the corresponding shells. The goal of this subsection is to analyze such conditions when $\varepsilon$ deformations with nontrivial $\mathrm{N}$-connection coefficients for 
the prime and target d-metrics can be related to new classes of solutions of motion heterotic string equations.

We denote nonholonomic $\varepsilon$-deformations of certain prime d-metric (75) into a target one (76) with polarizations (77) in the form $\stackrel{\circ}{\mathbf{g}} \rightarrow{ }_{\varepsilon} \mathbf{g}=\left({ }_{\varepsilon} g_{i},{ }_{\varepsilon} h_{a_{s}},{ }_{\varepsilon} N_{b_{s}}^{a_{s-1}}\right)$. The goal of this subsection is to compute the formulas for $\varepsilon$-deformations of prime d-metrics resulting in stationary solutions in equivalent nonholonomic 10-d gravity with Killing symmetries on $\partial_{t}$ and $\partial_{9}$.

The geometric constructions will be provided in detail for the 4-d configurations and then extended to higherdimensional shells. Deformations of $h$-components are characterized by ${ }_{\varepsilon} g_{i}=\stackrel{\circ}{g}_{i}\left(1+\varepsilon \chi_{i}\right)=e^{q\left(x^{k}\right)}$ resulting in a solution of the 2-d Laplace equation (39). For $q={ }^{0} q\left(x^{k}\right)+$ ${ }_{\varepsilon}{ }^{1} q\left(x^{k}\right)$ and ${ }_{h} \Upsilon={ }_{h}^{0} \Upsilon\left(x^{k}\right)+{ }_{h}^{\tilde{1}} \Upsilon\left(x^{k}\right)$, we compute the deformation polarization functions $\chi_{i}=e^{0} q 1{ }^{1} q / \stackrel{\circ}{g}_{i}{ }_{h}^{0} \Upsilon$. In this formula, we use certain generating and source functions as solutions of ${ }^{0} q^{\bullet \bullet}+{ }^{0} q^{\prime \prime}={ }^{0} \Upsilon$ and ${ }^{1} q^{\bullet \bullet}+{ }^{1} q^{\prime \prime}={ }^{1} \Upsilon$.

At the next step, we compute $\varepsilon$-deformations of $v$ components on the $s=0$ shell,

$$
\begin{aligned}
{ }_{\varepsilon} h_{3} & =h_{3}^{[0]}\left(x^{k}\right)-\frac{1}{4} \int \mathrm{d} y^{4} \frac{\left(\Phi^{2}\right)^{*}}{\Upsilon}=\left(1+\varepsilon \chi_{3}\right) \stackrel{\circ}{g}_{3}, \\
{ }_{\varepsilon} h_{4} & =-\frac{1}{4} \frac{\left(\Phi^{*}\right)^{2}}{\Upsilon^{2}}\left(h_{3}^{[0]}-\frac{1}{4} \int \mathrm{d} y^{4} \frac{\left(\Phi^{2}\right)^{*}}{\Upsilon}\right)^{-1} \\
& =\left(1+\varepsilon \chi_{4}\right) \stackrel{\circ}{g}_{4} ;
\end{aligned}
$$

Parametrizing the generation function,

$$
\Phi \rightarrow{ }_{\varepsilon} \Phi=\stackrel{\Phi}{\Phi}\left(x^{k}, y^{4}\right)\left[1+\varepsilon \chi\left(x^{k}, y^{4}\right)\right],
$$

and introducing this value in (79), one obtains

$$
\begin{gathered}
\chi_{3}=-\frac{1}{4 \stackrel{\circ}{g}_{3}} \int \mathrm{d} y^{4} \frac{\left(\stackrel{\circ}{ }^{2} \chi\right)^{*}}{\Upsilon} \text { and } \\
\int \mathrm{d} y^{4} \frac{\left(\Phi^{2}\right)^{*}}{\Upsilon}=4\left(h_{3}^{[0]}-\stackrel{\circ}{g}_{3}\right) .
\end{gathered}
$$

In result, we can compute $\chi_{3}$ for any deformation $\chi$ from a 2hypersurface $y^{4}=y^{4}\left(x^{k}\right)$. Such a hypersurface, in general, is defined in non-explicit form from $\Phi=\AA\left(x^{k}, y^{4}\right)$ when the integration function $h_{3}^{[0]}\left(x^{k}\right)$, the prime value $\stackrel{\circ}{g}_{3}\left(x^{k}\right)$, and the fraction $\left(\Phi^{2}\right)^{*} / \Upsilon$ satisfy the condition (81).

We can find the formula for hypersurface $\Phi\left(x^{k}, y^{4}\right)$ prescribing a value of $\Upsilon$. Introducing (64) into (79), one obtains

$$
\begin{aligned}
\chi_{4} & =2\left(\chi+\frac{\stackrel{\circ}{\Phi^{*}}}{*}\right)-\chi_{3} \\
& =2\left(\chi+\frac{\stackrel{\Phi}{\Phi}}{\Phi^{*}} \chi^{*}\right)+\frac{1}{4 \stackrel{\circ}{g}_{3}} \int \mathrm{d} y^{4} \frac{\left(\AA^{2} \chi\right)^{*}}{\Upsilon},
\end{aligned}
$$

i.e. we compute $\chi_{4}$ for any data $\left(\stackrel{\circ}{\Phi}, \stackrel{\circ}{g}_{3}, \chi\right)$. The formula for a compatible source is ${ }_{h} \Upsilon= \pm \Phi^{*} / 2 \sqrt{\left|g_{4}^{\circ} h_{3}^{[0]}\right|}$. It transforms
(81) into a 2-d hypersurface formula $y^{4}=y^{4}\left(x^{k}\right)$ defined in non-explicit form from

$$
\int \mathrm{d} y^{4} \stackrel{\circ}{\Phi}= \pm\left(h_{3}^{[0]}-\stackrel{\circ}{g}_{3}\right) / \sqrt{\left|\stackrel{\circ}{g_{4}} h_{3}^{[0]}\right| .}
$$

The $\varepsilon$-deformations of $\mathrm{N}$-connection coefficients $w_{i}=$

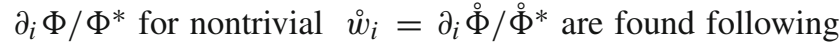
Eqs. (64) and (77), ${ }^{w} \chi_{i}=\frac{\partial_{i}(\chi \Phi)}{\partial_{i} \dot{\Phi}}-\frac{(\chi \Phi)^{*}}{\Phi^{*}}$, where there is no summation on index $i$. We can compute the deformations of the $n$-coefficients (we omit such details; see below for the necessary formulas).

In a similar way, the $\varepsilon$-deformations of ${ }^{1} v$-components on the $s=1$ shell are computed,

$$
\begin{aligned}
{ }_{\varepsilon} h_{5} & =h_{5}^{[0]}\left(x^{k}, y^{4}\right)+\int \mathrm{d} y^{6} \frac{\left({ }^{1} \Phi^{2}\right)^{* 1}}{4{ }_{1} \Upsilon}=\left(1+\varepsilon \chi_{5}\right) \stackrel{\circ}{g}_{5}, \\
{ }_{\varepsilon} h_{6} & =-\frac{\left({ }^{1} \Phi^{* 1}\right)^{2}}{{ }_{4} \Upsilon^{2}}\left(h_{5}^{[0]}+\frac{1}{4} \int \mathrm{d} y^{6} \frac{\left({ }^{1} \Phi^{2}\right)^{* 1}}{{ }_{1} \Upsilon}\right)^{-1} \\
& =\left(1+\varepsilon \chi_{6}\right) \stackrel{\circ}{g}_{6} .
\end{aligned}
$$

The first shell generation function is parameterized ${ }^{1} \Phi \rightarrow$ ${ }_{\varepsilon}^{1} \Phi={ }^{1} \Phi\left(x^{k}, y^{4}, y^{6}\right)\left[1+\varepsilon^{1} \chi\left(x^{k}, y^{4}, y^{6}\right)\right]$, resulting in

$$
\begin{aligned}
\chi_{5}=- & \frac{1}{4 \stackrel{\circ}{g}_{5}} \int \mathrm{d} y^{6} \frac{\left({ }^{1} \stackrel{\circ}{\Phi}^{2}{ }^{1} \chi\right)^{*_{1}}}{{ }_{1} \Upsilon} \text { and } \\
& \int \mathrm{d} y^{6} \frac{\left({ }^{1} \stackrel{\circ}{\Phi}^{2}\right)^{*_{1}}}{{ }_{1} \Upsilon}=4\left(h_{5}^{[0]}-\stackrel{\circ}{g}_{5}\right) .
\end{aligned}
$$

It is possible to compute $\chi_{5}$ for any deformation ${ }^{1} \chi$ from a 3-hypersurface $y^{6}=y^{6}\left(x^{k}, y^{4}\right)$ (for stationary solutions with Killing symmetries on $\partial_{t}$ and $\left.\partial_{5}\right)$. We note that, in general, such a hypersurface is defined in non-explicit form from ${ }^{1} \Phi={ }^{1} \Phi\left(x^{k}, y^{4}, y^{6}\right)$ when the integration function $h_{5}^{[0]}\left(x^{k}, y^{4}\right)$, the prime value $\stackrel{\circ}{g}_{5}\left(x^{k}, y^{4}\right)$ and the fraction $\left({ }^{1} \stackrel{\circ}{ }^{2}\right)^{*_{1}} /{ }_{1} \Upsilon$ satisfy a condition similar to (81).

We can find the formula for hypersurface ${ }^{1} \Phi\left(x^{k}, y^{4}, y^{6}\right)$ by prescribing a first shell value of effective source ${ }_{1} \Upsilon$. Similarly to (64) and (79), it is possible to generalize and compute

$$
\begin{aligned}
& \chi_{6}=2\left({ }^{1} \chi+\frac{1 \stackrel{\circ}{\Phi}}{1 \stackrel{\circ}{\Phi}^{* 1}}{ }^{1} \chi^{*_{1}}\right)-\chi_{5} \\
& =2\left({ }^{1} \chi+\frac{{ }^{1} \stackrel{\circ}{\Phi}^{1}}{{ }^{1} \Phi^{*_{1}}} \chi^{*_{1}}\right)+\frac{1}{4 \stackrel{\circ}{g}_{5}} \int \mathrm{d} y^{6} \frac{\left({ }^{1} \stackrel{\circ}{\Phi}^{2}{ }^{1} \chi\right)^{*_{1}}}{{ }_{1} \Upsilon},
\end{aligned}
$$

i.e. we compute $\chi_{6}$ for any data $\left({ }^{1} \stackrel{\circ}{\Phi}, \stackrel{g}{g}_{5},{ }^{1} \chi\right)$. One defines a first shell compatible source if ${ }_{1} \Upsilon= \pm{ }^{1} \stackrel{\circ}{ }^{* 1} / 2 \sqrt{\left|\stackrel{\circ}{g}_{6} h_{5}^{[0]}\right|}$. It generalizes (81) into a 2-d hypersurface formula $y^{6}=$ $y^{6}\left(x^{k}, y^{5}\right)$ which has to be computed in non-explicit form from $\int \mathrm{d} y^{6}{ }^{1} \stackrel{\circ}{\Phi}= \pm\left(h_{5}^{[0]}-\stackrel{\circ}{g}_{5}\right) / \sqrt{\left|\stackrel{\circ}{g}_{6} h_{5}^{[0]}\right|}$. On the first shell, the $\varepsilon$-deformations of $\mathrm{N}$-connection coefficients $w_{i_{1}}=$ $\partial_{i_{1}}{ }^{1} \Phi /{ }^{1} \Phi^{*_{1}}$ for nontrivial ${\stackrel{\circ}{i_{1}}}_{i_{1}}=\partial_{i_{1}}{ }^{1} \stackrel{\Phi}{\Phi} /{ }^{1} \stackrel{\circ}{\Phi}^{*_{1}}$ are 
${ }^{w} \chi_{i_{1}}=\frac{\partial_{i_{1}}\left({ }^{1} \chi^{1}{ }^{1} \stackrel{\circ}{\Phi}\right)}{\partial_{i_{1}}{ }^{1} \Phi}-\frac{\left({ }^{1} \chi{ }^{1}{ }^{1} \Phi\right)^{* 1}}{{ }^{1} \dot{\Phi}^{* 1}}$, where there is no summation on index $i_{1}$. We omit computations of deformations of the $n$-coefficients but we shall present necessary formulas below, see similar details in [43].

Summarizing the above formulas and extending for all shells $s=0,1,2,3$, we obtain such coefficients for $\varepsilon$ deformations of a prime metric (75) into a target stationary metric satisfying the equations of motion in heterotic supergravity:

$$
\begin{aligned}
& { }^{\varepsilon} g_{i}=\stackrel{\circ}{g}_{i}\left[1+\varepsilon \chi_{i}\right]=\left[1+\varepsilon e^{0} q 1{ }^{1} q / \stackrel{\circ}{g}_{i}{ }_{h}^{0} \Upsilon\right] \stackrel{\circ}{g}_{i} \\
& \text { as a solution of 2-d Poisson equations (39); } \\
& { }_{\varepsilon} h_{3}=\left[1+\varepsilon \chi_{3}\right] \stackrel{\circ}{g}_{3}=\left[1-\varepsilon \frac{1}{4 \stackrel{\circ}{g}_{3}} \int \mathrm{d} y^{4} \frac{\left(\stackrel{\circ}{ }^{2} \chi\right)^{*}}{\Upsilon}\right] \stackrel{\circ}{g}_{3} ; \\
& { }_{\varepsilon} h_{4}=\left[1+\varepsilon \chi_{4}\right] \stackrel{\circ}{g}_{4}=\left[1+\varepsilon\left(2\left(\chi+\frac{\stackrel{\circ}{\Phi}}{\dot{\circ}^{*}} \chi^{*}\right)\right.\right. \\
& \left.\left.+\frac{1}{4 \stackrel{\circ}{g}_{3}} \int \mathrm{d} y^{4} \frac{\left(\stackrel{\circ}{\Phi}^{2} \chi\right)^{*}}{\Upsilon}\right)\right] \stackrel{\circ}{g}_{4} \text {; } \\
& { }_{\varepsilon} n_{i}=\left[1+\varepsilon^{n} \chi_{i}\right] \stackrel{\circ}{n}_{i}=\left[1+\varepsilon \tilde{n}_{i} \int \mathrm{d} y^{4} \frac{1}{\Upsilon^{2}}\right. \\
& \left.\times\left(\chi+\frac{\stackrel{\circ}{\Phi}}{\stackrel{\circ}{\Phi}^{*}} \chi^{*}+\frac{5}{8 \stackrel{\circ}{g}_{3}} \frac{\left(\stackrel{\circ}{\Phi}^{2} \chi\right)^{*}}{\Upsilon}\right)\right] \stackrel{\circ}{n}_{i} \\
& { }_{\varepsilon} w_{i}=\left[1+\varepsilon^{w} \chi_{i}\right] \stackrel{\circ}{w}_{i}=\left[1+\varepsilon\left(\frac{\partial_{i}(\chi \stackrel{\circ}{\Phi})}{\partial_{i} \stackrel{\circ}{\Phi}}-\frac{(\chi \stackrel{\circ}{\Phi})^{*}}{\AA^{*}}\right)\right] \stackrel{\circ}{w}_{i},
\end{aligned}
$$

where $\tilde{n}_{i}\left(x^{k}\right)$ is a redefined integration function including contributions from the prime metric. On a shell $s$, these formulas are defined recurrently (we omit parameterizations of functions on shell coordinates because they can be determined in compatible form with indices and labels for shells),

$$
\begin{aligned}
& { }_{\varepsilon} h_{3+2 s}=\left[1+\varepsilon \chi_{3+2 s}\right] \stackrel{\circ}{g}_{3+2 s} \\
& =\left[1-\varepsilon \frac{1}{4 \stackrel{\circ}{g}_{3+2 s}} \int \mathrm{d} y^{4+2 s} \frac{\left({ }^{s} \stackrel{\circ}{\Phi}^{2} s \chi\right)^{* s}}{s \Upsilon}\right] \stackrel{\circ}{g}_{3+2 s} ; \\
& { }_{\varepsilon} h_{4+2 s}=\left[1+\varepsilon \chi_{4+2 s}\right]^{\circ} \stackrel{\circ}{4+2 s} \\
& =\left[1+\varepsilon\left(2\left({ }^{s} \chi+\frac{{ }^{s} \stackrel{\circ}{{ }^{\circ} *_{s}}}{s} \chi^{*_{s}}\right)\right.\right. \\
& \left.\left.+\frac{1}{4 \stackrel{\circ}{g}_{3+2 s}} \int \mathrm{d} y^{4+2 s} \frac{\left({ }^{s} \stackrel{\circ}{\Phi}^{2}{ }^{s} \chi\right)^{*_{1}}}{s \Upsilon}\right)\right] \stackrel{\circ}{g}_{4+2 s} ; \\
& { }_{\varepsilon} n_{i_{s}}=\left[1+\varepsilon^{n} \chi_{i_{s}}\right]{\stackrel{\circ}{i_{s}}} \\
& =\left[1+\varepsilon \tilde{n}_{i_{s}} \int \mathrm{d} y^{5+2 s} \frac{1}{{ }_{s} \Upsilon^{2}}\right.
\end{aligned}
$$

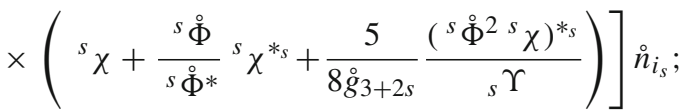

$$
\begin{aligned}
{ }_{\varepsilon} w_{i_{s}} & =\left[1+\varepsilon{ }^{w} \chi_{i_{s}}\right]{\stackrel{\circ}{i_{s}}} \\
& =\left[1+\varepsilon\left(\frac{\partial_{i_{s}}\left({ }^{s} \chi{ }^{s} \stackrel{\circ}{\Phi}\right)}{\partial_{i_{s}} s \stackrel{\circ}{\Phi}}-\frac{\left({ }^{s} \chi{ }^{s} \stackrel{\circ}{\Phi}\right)^{*_{s}}}{s \stackrel{\circ}{\Phi^{*}}}\right)\right]{\stackrel{\circ}{w_{i_{s}}}} .
\end{aligned}
$$

These formulas with $s=1,2,3$ allow us to parametrize all coefficients of vertical components of d-metrics and $\mathrm{N}$ connections. For small parametric deformations, the values $\chi,{ }^{s} \chi$ and $\Upsilon,{ }_{s} \Upsilon$ have to be considered as generating functions. The values with a "circle" are prescribed by a chosen prime solution (in our case, we can chose the 4-d Kerr metric embedded into a 10-d gravity string spacetime). Fixing a small value $\varepsilon$, we compute such deformations and prove their stability (see [38] and the references therein) for any stable prime solution.

The $\varepsilon$-deformed quadratic elements are written

$$
\begin{aligned}
\mathrm{d} s_{\varepsilon t}^{2}= & { }_{\varepsilon} g_{\alpha_{s} \beta_{s}}\left(x^{k}, y^{4}, y^{a_{1}}, y^{a_{2}}, y^{10}\right) \mathrm{d} u^{\alpha_{s}} \mathrm{~d} u^{\beta_{s}} \\
= & { }_{\varepsilon} g_{i}\left(x^{k}\right)\left[\left(\mathrm{d} x^{1}\right)^{2}+\left(\mathrm{d} x^{2}\right)^{2}\right]+{ }_{\varepsilon} h_{3}\left(x^{k}, y^{4}\right)[\mathrm{d} t \\
& \left.+{ }_{\varepsilon} n_{k}\left(x^{k}, y^{4}\right) \mathrm{d} x^{k}\right]^{2}+{ }_{\varepsilon} h_{4}\left(x^{k}, y^{4}\right) \\
& \times\left[\mathrm{d} y^{4}+{ }_{\varepsilon} w_{i}\left(x^{k}, y^{4}\right) \mathrm{d} x^{i}\right]^{2}+{ }_{\varepsilon} h_{5}\left(x^{k}, y^{4}, y^{6}\right) \\
& \times\left[\mathrm{d} y^{5}+{ }_{\varepsilon} n_{k_{1}}\left(x^{k}, y^{4}, y^{6}\right) \mathrm{d} x^{k_{1}}\right]^{2} \\
& +{ }_{\varepsilon} h_{6}\left(x^{k}, y^{4}, y^{6}\right)\left[\mathrm{d} y^{6}+{ }_{\varepsilon} w_{i_{1}}\left(x^{k}, y^{4}, y^{6}\right) \mathrm{d} x^{i_{1}}\right]^{2} \\
& +{ }_{\varepsilon} h_{7}\left(x^{k}, y^{4}, y^{a_{1}}, y^{8}\right)\left[\mathrm{d} y^{7}\right. \\
& \left.+{ }_{\varepsilon} n_{k_{2}}\left(x^{k}, y^{4}, y^{a_{1}}, y^{8}\right) \mathrm{d} x^{k_{2}}\right]^{2} \\
& +{ }_{\varepsilon} h_{8}\left(x^{k}, y^{4}, y^{a_{1}}, y^{8}\right)\left[\mathrm{d} y^{8}\right. \\
& +{ }_{\varepsilon} w_{i_{2}}\left(x^{k}, y^{4}, y^{a_{1}}, y^{8}\right) \mathrm{d} x^{i_{2}} \\
& +{ }_{\varepsilon} h_{9}\left(x^{k}, y^{4}, y^{a_{1}}, y^{a_{2}}, y^{10}\right) \\
& \times\left[\mathrm{d} y^{9}+{ }_{\varepsilon} n_{k_{3}}\left(x^{k}, y^{4}, y^{a_{1}}, y^{a_{2}}, y^{10}\right) \mathrm{d} x^{k_{3}}\right]^{2} \\
& +{ }_{\varepsilon} h_{10}\left(x^{k}, y^{4}, y^{a_{1}}, y^{a_{2}}, y^{10}\right) \\
& \times\left[\mathrm{d} y^{10}+{ }_{\varepsilon} w_{i_{3}}\left(x^{k}, y^{4}, y^{a_{1}}, y^{a_{2}}, y^{10}\right) \mathrm{d} x^{i_{3}}\right] .
\end{aligned}
$$

We can impose additional constraints in order to extract LCconfigurations as we considered in Sect. 2.3.8.

\section{Nonholonomic heterotic string deformations of the Kerr metric}

In this section, we study generic off-diagonal deformations and generalizations of the 4-d Kerr metric to new classes of exact solutions of equations of motion in heterotic string theory; see $[31,32,52,71]$. We prove that using the AFDM extended to models with almost-Kähler internal spaces, the Kerr solution can be constructed as a particular case by prescribing a corresponding class of generating and integration functions. In general, such solutions are with nontrivial cosmological constants and nonholonomically induced torsions. Imposing additional nonholonomic constraints, we can generate effective vacuum solutions and extract Levi-Civita configurations. A series of new classes of small parametric solu- 
tions when the Kerr metrics are nonholonomically deformed into general or ellipsoidal stationary configurations in fourdimensional gravity and/or extra dimensions are considered. We provide and study examples of generic off-diagonal metrics encoding nonlinear interactions with 3 -form and gaugelike fields, nonholonomically induced torsion effects and instanton configurations.

3.1 Preliminaries on the Kerr vacuum solution and nonholonomic variables

A 4-d ansatz

$\mathrm{d} s_{[0]}^{2}=Y^{-1} e^{2 h}\left(\mathrm{~d} \rho^{2}+\mathrm{d} z^{2}\right)-\rho^{2} Y^{-1} \mathrm{~d} t^{2}+Y(\mathrm{~d} \varphi+A \mathrm{~d} t)^{2}$

written in terms of three functions $(h, Y, A)$ on coordinates $x^{i}=(\rho, z)$ defines the Kerr solution of the vacuum Einstein equations (for rotating black holes) if we choose

$Y=\frac{1-\left(p \widehat{x}_{1}\right)^{2}-\left(q \widehat{x}_{2}\right)^{2}}{\left(1+p \widehat{x}_{1}\right)^{2}+\left(q \widehat{x}_{2}\right)^{2}}, \quad A=2 M \frac{q}{p} \frac{\left(1-\widehat{x}_{2}\right)\left(1+p \widehat{x}_{1}\right)}{1-\left(p \widehat{x}_{1}\right)-\left(q \widehat{x}_{2}\right)}$, $e^{2 h}=\frac{1-\left(p \widehat{x}_{1}\right)^{2}-\left(q \widehat{x}_{2}\right)^{2}}{p^{2}\left[\left(\widehat{x}_{1}\right)^{2}+\left(\widehat{x}_{2}\right)^{2}\right]}, \quad \rho^{2}=M^{2}\left(\widehat{x}_{1}^{2}-1\right)\left(1-\widehat{x}_{2}^{2}\right)$, $z=M \widehat{x}_{1} \widehat{x}_{2}$.

Some values $M=$ const and $\rho=0$ result in a horizon $\widehat{x}_{1}=0$ and the "north/south" segments of the rotation axis, $\widehat{x}_{2}=+1 /-1$. For further applications of the AFDM, we can write this prime solution in the form

$\mathrm{d} s_{[0]}^{2}=\left(\mathrm{d} x^{1}\right)^{2}+\left(\mathrm{d} x^{2}\right)^{2}-\rho^{2} Y^{-1}\left(\mathbf{e}^{3}\right)^{2}+Y\left(\mathbf{e}^{4}\right)^{2}$.

This is possible if the coordinates $x^{1}\left(\widehat{x}_{1}, \widehat{x}_{2}\right)$ and $x^{2}\left(\widehat{x}_{1}, \widehat{x}_{2}\right)$ are defined for any

$$
\begin{aligned}
& \left(\mathrm{d} x^{1}\right)^{2}+\left(\mathrm{d} x^{2}\right)^{2} \\
& =M^{2} e^{2 h}\left(\widehat{x}_{1}^{2}-\widehat{x}_{2}^{2}\right) Y^{-1}\left(\frac{\mathrm{d} \widehat{x}_{1}^{2}}{\widehat{x}_{1}^{2}-1}+\frac{\mathrm{d} \widehat{x}_{2}^{2}}{1-\widehat{x}_{2}^{2}}\right)
\end{aligned}
$$

and $y^{3}=t+\widehat{y}^{3}\left(x^{1}, x^{2}\right), y^{4}=\varphi+\widehat{y}^{4}\left(x^{1}, x^{2}, t\right)$. We can consider an $\mathrm{N}$-adapted basis $\mathbf{e}^{3}=\mathrm{d} t+\left(\partial_{i} \widehat{y}^{3}\right) \mathrm{d} x^{i}, \mathbf{e}^{4}=$ $\mathrm{d} y^{4}+\left(\partial_{i} \widehat{y}^{4}\right) \mathrm{d} x^{i}$, for some functions $\widehat{y}^{a}, a=3$, 4 , with $\partial_{t} \widehat{y}^{4}=-A\left(x^{k}\right)$.

The Kerr metric was intensively studied in the so-called Boyer-Lindquist coordinates $(r, \vartheta, \varphi, t)$, for $r=m_{0}(1+$ $\left.p \widehat{x}_{1}\right), \widehat{x}_{2}=\cos \vartheta$, which can be considered for applications of the AFDM. Such coordinates are expressed via parameters $p, q$ which are related to the total black hole mass, $m_{0}$ and the total angular momentum, $a m_{0}$, for the asymptotically flat, stationary, and antisymmetric Kerr spacetime. The formulas $m_{0}=M p^{-1}$ and $a=M q p^{-1}$ when $p^{2}+q^{2}=1$ imply $m_{0}^{2}-a^{2}=M^{2}$. In these variables, the metric (85) can be written

$$
\begin{aligned}
\mathrm{d} s_{[0]}^{2} & =\left(\mathrm{d} x^{1^{\prime}}\right)^{2}+\left(\mathrm{d} x^{2^{\prime}}\right)^{2}+\bar{A}\left(\mathbf{e}^{3^{\prime}}\right)^{2}+\left(\bar{C}-\bar{B}^{2} / \bar{A}\right)\left(\mathbf{e}^{4^{\prime}}\right)^{2}, \\
\mathbf{e}^{3^{\prime}} & =\mathrm{d} t+\mathrm{d} \varphi \bar{B} / \bar{A}=\mathrm{d} y^{3^{\prime}}-\partial_{i^{\prime}}\left(\widehat{y}^{3^{\prime}}+\varphi \bar{B} / \bar{A}\right) \mathrm{d} x^{i^{\prime}}, \\
\mathbf{e}^{4^{\prime}} & =\mathrm{d} y^{4^{\prime}}=\mathrm{d} \varphi,
\end{aligned}
$$

for any coordinate functions $x^{1^{\prime}}(r, \vartheta), x^{2^{\prime}}(r, \vartheta), y^{3^{\prime}}=$ $t+\widehat{y}^{3^{\prime}}(r, \vartheta, \varphi)+\varphi \bar{B} / \bar{A}, y^{4^{\prime}}=\varphi, \partial_{\varphi} \widehat{y}^{3^{\prime}}=-\bar{B} / \bar{A}$, for which $\left(\mathrm{d} x^{1^{\prime}}\right)^{2}+\left(\mathrm{d} x^{2^{\prime}}\right)^{2}=\Xi\left(\Delta^{-1} \mathrm{~d} r^{2}+\mathrm{d} \vartheta^{2}\right)$, and the coefficients are

$$
\begin{aligned}
& \bar{A}=-\Xi^{-1}\left(\Delta-a^{2} \sin ^{2} \vartheta\right), \\
& \bar{B}=\Xi^{-1} a \sin ^{2} \vartheta\left[\Delta-\left(r^{2}+a^{2}\right)\right], \\
& \bar{C}=\Xi^{-1} \sin ^{2} \vartheta\left[\left(r^{2}+a^{2}\right)^{2}-\Delta a^{2} \sin ^{2} \vartheta\right], \quad \text { and } \\
& \Delta=r^{2}-2 m_{0}+a^{2}, \quad \Xi=r^{2}+a^{2} \cos ^{2} \vartheta .
\end{aligned}
$$

We refer the reader to $[31,52,71]$ for main results and methods for stationary black hole solutions (the coordinates $\widehat{x}_{1}, \widehat{x}_{2}$ introduced above correspond to $x, y$, respectively, in chapter 4 of the first book).

The primed quadratic linear elements (85) (or (86))

$$
\stackrel{\circ}{g}_{1}=1, \stackrel{\circ}{g}_{2}=1, \stackrel{\circ}{h}_{3}=-\rho^{2} Y^{-1}, \stackrel{\circ}{h}_{4}=Y, \stackrel{\circ}{N}_{i}^{a}=\partial_{i} \widehat{y}^{a} \text {, or }
$$

$\stackrel{\circ}{g}_{1^{\prime}}=1,{\stackrel{\circ}{2^{\prime}}}_{2^{\prime}}=1,{\stackrel{\circ}{h^{\prime}}}_{3^{\prime}}=\bar{A}, \stackrel{\circ}{h}_{4^{\prime}}=\bar{C}-\bar{B}^{2} / \bar{A}$,

$\stackrel{\circ}{N}_{i^{\prime}}^{3}=\stackrel{\circ}{n}_{i^{\prime}}=-\partial_{i^{\prime}}\left(\widehat{y}^{3^{\prime}}+\varphi \bar{B} / \bar{A}\right), \stackrel{\circ}{N}_{i^{\prime}}^{4}=\stackrel{\circ}{w}_{i^{\prime}}=0$

define solutions of vacuum Einstein equations parametrized in the form (16) and (17) with zero sources. A straightforward application of the AFDM is possible if we consider a correspondingly $\mathrm{N}$-adapted system of coordinates instead of the "standard" prolate spherical, or Boyer-Lindquist system. Parametrizations (88) are most convenient for a straightforward application of the AFDM. This way we can generalize the solutions for coefficients depending on more than two coordinates, with non-Killing configurations and/or extra dimensions.

Working with general classes of stationary solutions generated by the AFDM, the Kerr vacuum solution in GR can be considered as a "degenerate" case of 4-d off-diagonal vacuum solutions determined by primary metrics with data (88) when the diagonal coefficients depend only on two "horizontal" $\mathrm{N}$-adapted coordinates. Such a metric contains off-diagonal terms induced by rotation frames in a form when the nonholonomically induced torsion is zero. In $\mathrm{N}$-adapted frames, further generic off-diagonal and extra dimension generalizations can be performed following standard geometric methods (see the following sections).

\subsection{Off-diagonal deformations of 4-d Kerr metrics} by heterotic string sources

Let us consider the coefficients (88) for the Kerr metric as the

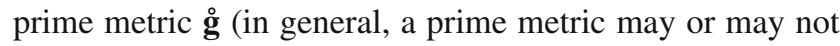


be an exact solution of the Einstein or other modified gravitational equations). Our goal is to construct nonholonomic deformations,

$$
\begin{aligned}
& \left(\mathbf{g}, \stackrel{\circ}{\mathbf{N}},{ }^{v} \stackrel{\circ}{\Upsilon}=0, \stackrel{\circ}{\Upsilon}=0\right) \rightarrow\left(\mathbf{g}, \mathbf{N},{ }_{h} \Upsilon\left(x^{k}\right) \rightarrow{ }_{h} \Lambda\right. \\
& \quad=\mathrm{const} \neq 0, \Upsilon \rightarrow \Lambda=\mathrm{const} \neq 0) ;
\end{aligned}
$$

see the sources (34), (37), and (19), respectively, with (21), when ${ }_{h} \Upsilon\left(x^{k}\right) \rightarrow{ }_{h} \Lambda={ }_{h}^{H} \Lambda+{ }_{h}^{F} \Lambda+{ }_{h}^{R} \Lambda, \Upsilon\left(x^{k}, y^{4}\right) \rightarrow$ $\Lambda={ }^{H} \Lambda+{ }^{F} \Lambda+{ }^{R} \Lambda$ and ${ }_{h}^{\phi} \Lambda={ }^{\phi} \Lambda=0$, as in (69). The main condition is that the target metric $\mathbf{g}$ is of type (74), which positively defines a torsionless off-diagonal solution of field equations in the 4-d gravity sector with sources determined from the heterotic string theory (12) with ansatz for the sources (38). The $\mathrm{N}$-adapted deformations of coefficients of metrics, frames, and sources are parametrized in the form

$$
\begin{aligned}
& {\left[\stackrel{\circ}{g}_{i}, \stackrel{\circ}{h}_{a}, \stackrel{\circ}{w}_{i}, \stackrel{\circ}{n}_{i}\right] \rightarrow\left[g_{i}=\eta_{i} \stackrel{\circ}{g}_{i}, h_{3}=\eta_{3} \stackrel{\circ}{h}_{3}, h_{4}\right.} \\
& \left.\quad=\eta_{4} \stackrel{\circ}{h}_{4}, w_{i}=\stackrel{\circ}{w}_{i}+{ }^{\eta} w_{i}, n_{i}=\stackrel{\circ}{n}_{i}+{ }^{\eta} n_{i}, \stackrel{\circ}{w}_{i}=0\right], \\
& \quad{ }_{h} \Upsilon=\Upsilon={ }_{K} \Lambda=-5\left[\left({ }^{H} s\right)^{2}+n_{F}\left({ }^{F} s\right)^{2}\left({ }^{R} s\right)^{2}\right], \check{\Phi}^{2} \\
& \quad=\exp \left[\check{\varpi}\left(x^{k^{\prime}}, y^{4}\right)\right], \stackrel{\circ}{h}_{3}=h_{3}^{(0)}, \stackrel{\circ}{h}_{4}=-\check{\Upsilon}^{2} /{ }_{K} \Lambda^{2}
\end{aligned}
$$

The primes $\stackrel{\circ}{g}_{i}, \stackrel{\circ}{h}_{a}, \stackrel{\circ}{w}_{i}, \stackrel{\circ}{n}_{i}$ (88) are given by coefficients depending only on $\left(x^{k \prime}\right)$. The general deformations of the Kerr solution determined by generating function and extradimensional string sources with ansatz resulting in cosmological constants described in terms of polarization functions, where $\left|\eta_{4^{\prime}}\right|$, may not have a smooth limit to 1 ,

$$
\begin{aligned}
& \eta_{3^{\prime}}=1-\left(\check{\Phi}^{2}\right) /{ }_{K} \Lambda \stackrel{\circ}{h}_{3}, \quad \eta_{4^{\prime}}=\left(\check{\Phi}^{*}\right)^{2} /\left({ }_{K} \Lambda\right)^{2} \eta_{3^{\prime}} \stackrel{\stackrel{\circ}{h}_{3}}{ }, \\
& { }^{\eta} w_{i^{\prime}}=\partial_{i^{\prime}} \check{A},{ }^{\eta} n_{k^{\prime}}=\partial_{k^{\prime}}{ }^{\eta} n\left(x^{i^{\prime}}\right),
\end{aligned}
$$

where the coefficient $1 / 4$ was introduced in $\breve{\Phi}$ and the function $\breve{A}$ has any value for which $\partial_{i^{\prime}} \check{\Phi} / \breve{\Phi}^{*}=\partial_{i^{\prime}} \check{A}$.

Summarizing the above formulas, we obtain the quadratic element

$$
\begin{aligned}
\mathrm{d} s_{K \eta 4 d}^{2}= & \check{g}_{\alpha \beta}\left(x^{k}, y^{4}\right) \mathrm{d} u^{\alpha} \mathrm{d} u^{\beta}=\eta_{i} \stackrel{\circ}{g}_{i}\left(\mathrm{~d} x^{i}\right)^{2}+\eta_{a} \stackrel{\circ}{g} a_{a}\left(\mathbf{e}^{a}\right)^{2} \\
= & e^{q\left[{ }_{K} \Lambda\right]}\left[\left(\mathrm{d} x^{1 \prime}\right)^{2}+\left(\mathrm{d} x^{2 \prime}\right)^{2}\right]+\left(\bar{A}-\frac{\check{\Phi}^{2}}{K^{\Lambda}}\right) \\
& \times\left[\mathrm{d} y^{3^{\prime}}+\left(\partial_{k^{\prime}} \eta^{\eta} n\left(x^{i^{\prime}}\right)-\partial_{k^{\prime}}\left(\widehat{y}^{3^{\prime}}+\varphi \frac{\bar{B}}{\bar{A}}\right)\right) \mathrm{d} x^{k^{\prime}}\right]^{2} \\
& \left.+\frac{\left(\check{\Phi}^{*}\right)^{2}}{{ }_{K} \Lambda\left({ }_{K} \Lambda \bar{A}-\check{\Phi}^{2}\right)}\left(\bar{C}-\frac{\bar{B}^{2}}{\bar{A}}\right)\left[\mathrm{d} \varphi+\partial_{i^{\prime}} \check{A}\right) \mathrm{d} x^{i^{\prime}}\right]^{2},
\end{aligned}
$$

where $w_{i^{\prime}}={\stackrel{\circ}{i^{\prime}}}_{i^{\prime}}+{ }^{\eta} w_{i^{\prime}}=\partial_{i^{\prime}}(\eta \widetilde{A}[\varpi]), n_{k^{\prime}}={\stackrel{\circ}{k^{\prime}}}+{ }^{\eta} n_{k^{\prime}}=$ $\partial_{k^{\prime}}\left(-\widehat{y}^{3^{\prime}}+\varphi \bar{B} / \bar{A}+{ }^{\eta} n\right)$ and $q$ is a solution of

$q^{\bullet \bullet}+q^{\prime \prime}=2_{K} \Lambda$.

We used the important relation ${\stackrel{\circ}{h} 3^{\prime}}_{4^{\prime}}=\overline{A C}-\bar{B}^{2}$ and emphasize that it is possible to take any function ${ }^{\eta} n\left(x^{k}\right)$.
The solutions (90) are for stationary LC-configurations determined by off-diagonal heterotic string gravity effects on Kerr black holes when the new class of spacetimes come with Killing symmetry on $\partial / \partial y^{3^{\prime}}$ and generic dependence on three (from maximally four) coordinates, $\left(x^{i^{\prime}}(r, \vartheta), \varphi\right)$. Similar solutions were constructed and studied in massive gravity with extra dimensions [43]. Off-diagonal modifications are possible for any nontrivial values of $\check{\Phi}$ and any small constants ${ }^{H} \Lambda,{ }^{F} \Lambda,{ }^{R} \Lambda$. The solutions depend on the type of generating function $\breve{\Phi}\left(x^{i^{\prime}}, \varphi\right)$ we fix in order to suit certain experimental/observational data for fixed systems of reference/coordinates. These can be re-parameterized for an effective ${ }_{K} \Lambda$, which should also be compatible with experimental data. In such variables, we can mimic stationary heterotic string gravity effects by off-diagonal configurations in GR with integration parameters which should also be fixed by additional assumptions on symmetries of interactions. See Sect. 3.3 for ellipsoid configurations and details on parametric Killing symmetries in Refs. [33-35].

\subsubsection{Nonholonomically string induced torsion for Kerr metrics in the 4-d sector}

If we do not impose the LC-conditions (17), a nontrivial source $\Upsilon \rightarrow \Lambda$ from heterotic string gravity induces stationary configurations with nontrivial d-torsion (7). The torsion coefficients are determined by metrics of type (59) with ${ }_{h} \Upsilon=\Upsilon={ }_{K} \Lambda$ as in (89) and parametrizations of coefficients and coordinates distinguishing the prime data for a Kerr metric (88). Such solutions can be written in the form

$$
\begin{aligned}
\mathrm{d} s^{2}= & e^{q}\left[\left(\mathrm{~d} x^{1^{\prime}}\right)^{2}+\left(\mathrm{d} x^{2^{\prime}}\right)^{2}\right] \\
& +\left(\bar{A}-\frac{\Phi^{2}}{{ }_{K} \Lambda}\right)\left[\mathrm{d} y^{3^{\prime}}+\left({ }_{1} n_{k^{\prime}}\left(x^{i^{\prime}}\right)\right.\right. \\
& +{ }_{2} n_{k^{\prime}}\left(x^{i^{\prime}}\right) \int \mathrm{d} y^{4}\left(\Phi^{2}\right)^{*}\left({ }_{K} \Lambda \bar{A}-\Phi^{2}\right)^{-5 / 2} \\
& \left.\left.-\partial_{k^{\prime}}\left(\widehat{y}^{3^{\prime}}+\varphi \frac{\bar{B}}{\bar{A}}\right)\right) \mathrm{d} x^{k^{\prime}}\right]^{2} \\
& -\frac{\left(\Phi^{*}\right)^{2}}{{ }_{K} \Lambda} \frac{\overline{A C}-\bar{B}^{2}}{{ }_{K} \Lambda \bar{A}-\Phi^{2}}\left[\mathrm{~d} \varphi+\frac{\partial_{i^{\prime}} \Phi}{\partial_{\varphi} \Phi} \mathrm{d} x^{i^{\prime}}\right]^{2},
\end{aligned}
$$

where the generating function $\Phi\left(x^{i^{\prime}}, \varphi\right)$ is not subject to any integrability conditions. Nontrivial stationary off-diagonal torsion effects may result in additional effective rotations if the integration function ${ }_{2} n_{k} \neq 0$. Considering two different classes of off-diagonal solutions (91) and (90), we can study if heterotic string corrections to GR can have a nonholonomically induced torsion or if such effects are characterized by additional nonholonomic constraints as in GR (for zero torsion).

It should be noted that configurations of type (91) can be constructed in various theories with noncommutative and 
commutative variables. We can consider warped and trapped brane type variables in string, Finsler-like and/or HořavaLifshitz theories $[24,38,51,55,56]$ when nonholonomically induced torsion effects play a substantial role.

\subsubsection{Small modifications of Kerr metrics and effective string sources}

It is not clear what physical meaning general deformations of the Kerr metric described by metrics of type (91) may have. We can choose certain subclasses of nonholonomic distributions describing stationary $\varepsilon$-deformations described by Eqs. (83). Using the Kerr solution (88) as a primary metric with assumptions for the string sources, we compute small deformations into $\mathrm{d}$-metric and $\mathrm{N}$-connection coefficients,

$$
\begin{aligned}
\mathrm{d} s_{K \varepsilon 4 d}^{2}= & \check{g}_{\alpha \beta}\left(x^{k}, y^{4}\right) \mathrm{d} u^{\alpha} \mathrm{d} u^{\beta}=\left[1+\varepsilon \chi_{i}\left(x^{k}\right)\right] g_{i}\left(\mathrm{~d} x^{i}\right)^{2} \\
& +\left[1+\varepsilon \chi_{a}\left(x^{k}, y^{4}\right)\right] g_{a}^{\circ}\left(\mathbf{e}^{a}\right)^{2} .
\end{aligned}
$$

The $\varepsilon$-deformations are computed thus:

$$
\begin{aligned}
& { }^{\varepsilon} g_{i}=\stackrel{\circ}{g}_{i}\left[1+\varepsilon \chi_{i}\right]=\left[1+\varepsilon e^{0} q 1{ }^{1} q /{ }_{K} \Lambda\right] \stackrel{\circ}{g}_{i} \\
& \text { as a solution of 2-d Poisson equations (39); } \\
& { }_{\varepsilon} h_{3}=\left[1+\varepsilon \chi_{3}\right] \stackrel{\circ}{g}_{3}=\left[1-\varepsilon \frac{\stackrel{\circ}{ }^{2} \chi}{4 \bar{A}_{K} \Lambda}\right] \stackrel{\circ}{g}_{3} \text {; } \\
& { }_{\varepsilon} h_{4}=\left[1+\varepsilon \chi_{4}\right] \stackrel{\circ}{g}_{4} \\
& =\left[1+\varepsilon\left(2\left(\chi+\frac{\stackrel{\circ}{\Phi}}{\Phi^{*}} \chi^{*}\right)+\frac{\stackrel{\circ}{\Phi}^{2} \chi}{4 \bar{A}_{K} \Lambda}\right)\right] \stackrel{\circ}{g}_{4} ; \\
& { }_{\varepsilon} n_{i}=\left[1+\varepsilon^{n} \chi_{i}\right] \stackrel{\circ}{n}_{i} \\
& =\left[1+\varepsilon \widetilde{n}_{i} \int \mathrm{d} y^{4}\left(\chi+\frac{\stackrel{\Phi}{\circ}}{\Phi^{*}} \chi^{*}+\frac{5\left(\AA^{2} \chi\right)^{*}}{8 \bar{A}_{K} \Lambda}\right)\right] \stackrel{\circ}{i}_{i} ; \\
& { }_{\varepsilon} w_{i}=\left[1+\varepsilon^{w} \chi_{i}\right] \stackrel{\circ}{w}_{i}=\left[1+\varepsilon\left(\frac{\partial_{i}(\chi \stackrel{\circ}{\Phi})}{\partial_{i} \stackrel{\circ}{\Phi}}-\frac{(\chi \stackrel{\circ}{\Phi})^{*}}{\AA^{*}}\right)\right] \stackrel{\circ}{w}_{i},
\end{aligned}
$$

where $\stackrel{\circ}{g}_{1^{\prime}}=1, \stackrel{\circ}{g}_{2^{\prime}}=1, \stackrel{\circ}{h}_{3^{\prime}}=\bar{A}, \stackrel{\circ}{h}_{4^{\prime}}=\bar{C}-\bar{B}^{2} / \bar{A},{\stackrel{\circ}{N^{\prime}}}_{i^{\prime}}^{3}=$ ${\stackrel{\circ}{i^{\prime}}}=-\partial_{i^{\prime}}\left(\widehat{y}^{3^{\prime}}+\varphi \bar{B} / \bar{A}\right)$ are kept as above but a coordinate transform is performed in order to have ${\stackrel{\circ}{N^{\prime}}}^{4}=\stackrel{\circ}{w}_{i^{\prime}} \neq 0$. We use polarization functions linearized on $\varepsilon$,

$$
\begin{gathered}
\eta_{i}=1+\varepsilon \chi_{i}\left(x^{k}\right), \quad \eta_{a}=1+\varepsilon \chi_{a}\left(x^{k}, y^{4}\right) ; \\
\quad \text { and for the coefficients of N-connection, } \\
{ }^{n} \eta_{i}=1+\varepsilon{ }^{n} \eta_{i}\left(x^{k}, y^{4}\right), \quad{ }^{w} \eta_{i}=1+\varepsilon^{w} \chi_{i}\left(x^{k}, y^{4}\right) .
\end{gathered}
$$

Summarizing the above for the 4-d $\varepsilon$-configurations, we obtain the quadratic element

$$
\begin{aligned}
& \mathrm{d} s_{K \varepsilon 4 d}^{2}=\left(1+\varepsilon e^{0} q \frac{{ }^{1} q}{{ }_{K} \Lambda}\right)\left[\left(\mathrm{d} x^{1 \prime}\right)^{2}+\left(\mathrm{d} x^{2 \prime}\right)^{2}\right] \\
& \quad+\left(\bar{A}-\varepsilon \frac{\AA^{2} \chi}{4{ }_{K} \Lambda}\right)\left[\mathrm{d} y^{3^{\prime}}-\left[1+\varepsilon \widetilde{n}_{i} \int \mathrm{d} y^{4}\right.\right. \\
& \left.\left.\quad \times\left(\chi+\frac{\Phi^{\circ}}{\Phi^{*}} \chi^{*}+\frac{5\left(\AA^{2} \chi\right)^{*}}{8 \bar{A}_{K} \Lambda}\right)\right] \partial_{k^{\prime}}\left(\widehat{y}^{3^{\prime}}+\varphi \frac{\bar{B}}{\bar{A}}\right) \mathrm{d} x^{k^{\prime}}\right]^{2}
\end{aligned}
$$

$$
\begin{aligned}
& +\left[1+\varepsilon\left(2\left(\chi+\frac{\stackrel{\circ}{\Phi}}{\Phi^{*}} \chi^{*}\right)+\frac{\stackrel{\circ}{ }^{2} \chi}{4 \bar{A}_{K} \Lambda}\right)\right] \\
& \times\left(\bar{C}-\frac{\bar{B}^{2}}{\bar{A}}\right)\left[\mathrm{d} \varphi+\left[1+\varepsilon\left(\frac{\partial_{i}(\chi \stackrel{\circ}{\Phi})}{\partial_{i} \stackrel{\circ}{\Phi}}-\frac{(\chi \stackrel{\circ}{\Phi})^{*}}{\Phi^{*}}\right)\right] \stackrel{\circ}{w}_{i}\right]^{2} \text {. }
\end{aligned}
$$

In general, these heterotic string deformations of the Kerr metric are nonholonomically induced torsion coefficients, linear in $\varepsilon$.

We can impose additional constraints on $\chi, \widetilde{n}_{i}$ and $\Phi$ which allows us to extract LC-configurations. The corresponding $\varepsilon$-deformed analog of the metric (90) can be written

$$
\begin{aligned}
& \mathrm{d} s_{K \eta 4 d}^{2}=\left(1+\varepsilon e^{0} q \frac{{ }^{1} q}{{ }_{K} \Lambda}\right)\left[\left(\mathrm{d} x^{1 \prime}\right)^{2}+\left(\mathrm{d} x^{2 \prime}\right)^{2}\right] \\
& +\left(\bar{A}-\varepsilon \frac{\AA^{2} \chi}{{ }_{K} \Lambda}\right)\left[\mathrm{d} y^{3^{\prime}}+\left(\varepsilon \partial_{k^{\prime}} \chi_{n}\left(x^{i^{\prime}}\right)\right.\right. \\
& \left.\left.-\partial_{k^{\prime}}\left(\widehat{y}^{3^{\prime}}+\varphi \frac{\bar{B}}{\bar{A}}\right)\right) \mathrm{d} x^{k^{\prime}}\right]^{2} \\
& +\left[1+\varepsilon\left(2\left(\chi+\frac{\stackrel{\circ}{\Phi}}{\Phi^{*}} \chi^{*}\right)+\frac{\stackrel{\Phi}{ }^{2} \chi}{4 \bar{A}_{K} \Lambda}\right)\right] \\
& \left.\times\left(\bar{C}-\frac{\bar{B}^{2}}{\bar{A}}\right)\left[\mathrm{d} \varphi+\varepsilon \partial_{i^{\prime}} \check{A}\right) \mathrm{d} x^{i^{\prime}}\right]^{2},
\end{aligned}
$$

where $\chi$ defines a deformed generating function ${ }^{\varepsilon} \Phi=$ $\Phi\left(x^{k}, y^{4}\right)\left[1+\varepsilon \chi\left(x^{k}, y^{4}\right)\right]$ as in Eq. (80) but subjected to the condition

$\varepsilon \partial_{i^{\prime}} \check{A}=\partial_{i^{\prime}}\left({ }^{\varepsilon} \Phi\right) /\left({ }^{\varepsilon} \Phi\right)^{*}$,

which together with $\chi_{n}\left(x^{i^{\prime}}\right)$ are chosen to result in zero nonholonomically induced torsion.

\subsection{String induced ellipsoidal 4-d deformations of the Kerr} metric

We provide some examples of how the Kerr primary data (88) are nonholonomically deformed by heterotic string interactions into target generic off-diagonal solutions of vacuum and non-vacuum Einstein equations for the canonical dconnection and/or the Levi-Civita connection. Generic offdiagonal metrics of type (92) can be parameterized as ellipsoidal deformations of the Kerr metric on a small eccentricity parameter $\varepsilon$.

\subsubsection{Ellipsoidal configurations with string induced cosmological constant}

Let us construct solutions for $\varepsilon$-deformations of type (92) with ellipsoidal configurations. We choose a generating function $\chi_{3^{\prime}}$, when the constraint $h_{3^{\prime}}=0$ defines a stationary 
rotoid configuration (different from the ergo sphere for the Kerr solutions): We prescribe

$\chi_{3^{\prime}}=\frac{\stackrel{\Phi}{ }^{2} \chi}{4 \bar{A}_{K} \Lambda}=2 \underline{\zeta} \sin \left(\omega_{0} \varphi+\varphi_{0}\right)$,

for constant parameters $\underline{\zeta}, \omega_{0}$, and $\varphi_{0}$, where the values

$$
\begin{aligned}
& \bar{A}(r, \vartheta)\left[1+\varepsilon \chi_{3^{\prime}}(r, \vartheta, \varphi)\right]=\widehat{A}(r, \vartheta, \varphi) \\
& \quad=-\Xi^{-1}\left(\widehat{\Delta}-a^{2} \sin ^{2} \vartheta\right) \text { and } \widehat{\Delta}(r, \varphi)=r^{2}-2 m(\varphi)+a^{2},
\end{aligned}
$$

are considered as $\varepsilon$-deformations of Kerr coefficients (87). We get an effective "anisotropically polarized" mass

$m(\varphi)=m_{0} /\left(1+\varepsilon \underline{\zeta} \sin \left(\omega_{0} \varphi+\varphi_{0}\right)\right)$.

As regards the result, the condition $h_{3}=0$, i.e. ${ }^{\varphi} \Delta(r, \varphi, \varepsilon)=$ $a^{2} \sin ^{2} \vartheta$, states an ellipsoidal "deformed horizon"

$r(\vartheta, \varphi)=m(\varphi)+\left(m^{2}(\varphi)-a^{2} \sin ^{2} \vartheta\right)^{1 / 2}$.

If $a=0$, we obtain the parametric formula for an ellipse with eccentricity $\varepsilon, r_{+}=\frac{2 m_{0}}{1+\varepsilon \zeta \sin \left(\omega_{0} \varphi+\varphi_{0}\right)}$. Such configurations correspond to the generating function

$\chi=\frac{8 \widetilde{\zeta}_{\bar{A}}{ }_{K} \Lambda}{\Phi^{2}} \sin \left(\omega_{0} \varphi+\varphi_{0}\right)$

determined by effective heterotic string source ${ }_{K} \Lambda$, as follows from (93).

If the anholonomy coefficients (4) computed for (92) are not trivial for $w_{i}$ and $n_{k}={ }_{1} n_{k}$, the generated solutions cannot be diagonalized via coordinate transforms.

The corresponding nonholonomically deformed 4-d spacetimes have one Killing symmetry on $\partial / \partial y^{3^{\prime}}$. For small $\varepsilon$, the singularity at $\Xi=0$ is "hidden" under ellipsoidal deformed horizons if $m_{0} \geq a$. Similarly to the Kerr solution, there are $\varphi$-deformed cases for both the event horizon,

$r_{+}=m(\varphi)+\left(m^{2}(\varphi)-a^{2} \sin ^{2} \vartheta\right)^{1 / 2}$,

and the Cauchy horizon,

$r_{-}=m(\varphi)-\left(m^{2}(\varphi)-a^{2} \sin ^{2} \vartheta\right)^{1 / 2}$,

which are effectively embedded into an off-diagonal background determined by $\mathrm{N}$-coefficients. Using an ellipsoid type generating function (95) in (92), we construct a class of generic off-diagonal solutions of effective Einstein equations with heterotic string gravity effective cosmological constant ${ }_{K} \Lambda$, which in its turn can be related to arbitrary sources via a redefinition of the generating functions (see Eqs. (70) and (37)) adapted to $\varepsilon$-deformations. The corresponding quadratic line elements are

$$
\begin{aligned}
\mathrm{d} s_{K \eta 4 d}^{2}= & \left(1+\varepsilon e^{0} q \frac{{ }^{1} q}{{ }_{K} \Lambda}\right)\left[\left(\mathrm{d} x^{1 \prime}\right)^{2}+\left(\mathrm{d} x^{2 \prime}\right)^{2}\right]+\bar{A}[1 \\
& \left.-2 \varepsilon \widetilde{\zeta} \sin \left(\omega_{0} \varphi+\varphi_{0}\right)\right]\left[\mathrm{d} y^{3^{\prime}}\right.
\end{aligned}
$$

$$
\begin{aligned}
& \left.+\left(\varepsilon \partial_{k^{\prime}} \chi_{n}\left(x^{i^{\prime}}\right)-\partial_{k^{\prime}}\left(\widehat{y}^{3^{\prime}}+\varphi \frac{\bar{B}}{\bar{A}}\right)\right) \mathrm{d} x^{k^{\prime}}\right]^{2} \\
& +\left[1+\varepsilon\left(\frac{\left(8 \bar{A}_{K} \Lambda+\AA^{2}\right) \tilde{\zeta}}{4_{K} \Lambda \AA^{2}} \sin \left(\omega_{0} \varphi+\varphi_{0}\right)\right.\right. \\
& \left.\left.-\frac{16 \tilde{\zeta} \omega_{0} \bar{A}_{K} \Lambda}{3 \dot{\Phi}^{2}} \cos \left(\omega_{0} \varphi+\varphi_{0}\right)\right)\right] \\
& \left.\times\left(\bar{C}-\frac{\bar{B}^{2}}{\bar{A}}\right)\left[\mathrm{d} \varphi+\varepsilon \partial_{i^{\prime}} \check{A}\right) \mathrm{d} x^{i^{\prime}}\right]^{2}
\end{aligned}
$$

The new classes of $\varepsilon$-deformed solutions determine Kerrlike black hole solutions with additional dependencies on the variable $\varphi$ of certain diagonal and off-diagonal coefficients of the metric. There is an obvious anisotropy in the angle $\varphi$. The values $\tilde{\zeta}$ and $\omega_{0}$ have to be chosen in accordance with experimental data. The function $\stackrel{\Phi}{\Phi}$ depends on corresponding frame distributions for the prime metric. Fixing $a=0$ for a $\varepsilon \neq 0$, we get ellipsoidal deformations of the Schwarzschild black holes. We studied these constructions in detail in [38]; see also the references therein on the stability and interpretation of such solutions with both commutative and/or noncommutative deformation parameters. In general, a black hole/ellipsoid interpretation is not possible for "non-small" $N$-deformations of the Kerr metric. For certain embeddings, we can generate black hole-like configurations with deformed horizons and locally anisotropic polarized physical constants.

\subsubsection{Ellipsoid Kerr-de Sitter configurations in $R^{2}$ and heterotic string gravity}

The first examples of generic off-diagonal ellipsoid-solitonic deformations of similar Kerr-Sen black holes were constructed in [72]. Recently, asymptotically de Sitter solutions with spherical symmetry for $R^{2}$ gravity were studied in [14] and the nonholonomic geometric off-diagonal evolution of such metrics was analyzed in [70]. In this section, we show that those constructions can be related to the 4-d part of heterotic string MGTs.

We consider a prime 4-d metric

$$
\begin{aligned}
\mathrm{d} \bar{s}^{2}= & \frac{3 \lambda}{2 \varsigma^{2}}\left\{\left(1-\frac{M}{r}-\lambda r^{2}\right)^{-1} \mathrm{~d} r^{2}+r^{2} \mathrm{~d} \theta^{2}\right. \\
& \left.+r^{2} \sin \theta \mathrm{d} \varphi^{2}-\left(1-\frac{M}{r}-\lambda r^{2}\right) \mathrm{d} t^{2}\right\},
\end{aligned}
$$

which for

$$
\begin{aligned}
e^{\sqrt{1 / 3} \phi} & =\frac{3 \lambda}{2 \varsigma^{2}}=\frac{1}{8 \varsigma^{2}} R \text { and } \\
\bar{g}_{\mu \nu} & =e^{\sqrt{1 / 3} \phi} g_{\mu \nu}=\frac{R}{8 \varsigma^{2}} g_{\mu \nu}, R \neq 0,
\end{aligned}
$$


defines exact solutions with spherical symmetry in $R^{2}$ gravity, for equations $\bar{R}_{\mu \nu}=2 \varsigma^{2} \bar{g}_{\mu \nu}$. The effective cosmological constant $\varsigma^{2}$ is usually related to nonlinear scalar fields/dilaton-like interactions in effective Einstein gravity resulting from $R^{2}$ gravity. In our model of heterotic string gravity, we can choose

$2 \varsigma^{2}={ }_{K} \Lambda$

and study quadratic gravity 4-d models determined by heterotic string effective sources. The metric (97) describes asymptotically de Sitter solutions with $\lambda>0$ and $R \neq 0$. Introducing new 4-d coordinates,

$$
\begin{aligned}
& \tilde{x}^{1^{\prime}}(r)=\sqrt{\left|\frac{3 \lambda}{2}\right|} \frac{1}{\zeta} \int \mathrm{d} r\left(1-\frac{M}{r}-\lambda r^{2}\right)^{-1 / 2}, \\
& \widetilde{x}^{2^{\prime}}=\theta, y^{3^{\prime}}=\varphi, y^{4^{\prime}}=t ; \\
& \stackrel{\circ}{g}_{1^{\prime}}=1, \underline{\stackrel{g}{g}}_{2^{\prime}}\left(\widetilde{x}^{1^{\prime}}\right)=r^{2}\left(\widetilde{x}^{1^{\prime}}\right), \quad \stackrel{\circ}{h}_{3^{\prime}}=r^{2}\left(\widetilde{x}^{1^{\prime}}\right) \sin \left(x^{2^{\prime}}\right) \text {, } \\
& {\stackrel{\circ}{h^{\prime}}}=-\left(1-\frac{M}{r\left(\widetilde{x^{\prime}}\right)}+\lambda r^{2}\left(\widetilde{x}^{1^{\prime}}\right)\right) \text {, }
\end{aligned}
$$

the metric (97) is written as a "prime" metric

$$
\begin{aligned}
& \mathrm{d} s^{2}=\underline{g}_{\alpha^{\prime} \beta^{\prime}}\left(\widetilde{x^{k^{\prime}}}\right) \mathrm{d} u^{\alpha^{\prime}} \mathrm{d} u^{\beta^{\prime}}=\stackrel{\circ}{g}_{1^{\prime}}\left(\mathrm{d} \widetilde{x}^{1^{\prime}}\right)^{2}+\underline{g}_{2^{\prime}}\left(\widetilde{1^{\prime}}\right)\left(\mathrm{d} \widetilde{x}^{\prime}\right)^{2} \\
& +\underline{\stackrel{h}{h}}_{3^{\prime}}\left(\widetilde{x}^{1^{\prime}}, \widetilde{x}^{2^{\prime}}\right)\left(\mathrm{d} y^{3^{\prime}}\right)^{2}+\underline{\circ}_{4^{\prime}}\left(\widetilde{x}^{1^{\prime}}\right)\left(\mathrm{d} y^{4^{\prime}}\right)^{2} \text {, }
\end{aligned}
$$

for some constants $M, \lambda$ and $u^{\alpha}=\left(\widetilde{x}^{k^{\prime}}, y^{a}\right)$. In order to work with a "formal" off-diagonal metric of type (75) with nontrivial values $\stackrel{\circ}{h}_{a}^{*}, \stackrel{\circ}{w}_{i}$ and $\stackrel{\circ}{n}_{i}$, but $\stackrel{\circ}{W}_{\beta \gamma}^{\alpha}\left(\widetilde{u}^{\mu}\right)=0$, see (4), we consider a coordinate transform $u^{\alpha^{\prime}}=u^{\alpha^{\prime}}\left(u^{\alpha}\right)$ with $\varphi=$ $\varphi\left(y^{4}, \widetilde{x}^{k}\right)$ and $t=t\left(y^{3}, \widetilde{x}^{k}\right)$, where

$$
\begin{gathered}
\mathrm{d} t=\frac{\partial t}{\partial y^{3}}\left[\mathrm{~d} y^{3}+\left(\partial_{3} t\right)^{-1}\left(\widetilde{\partial}_{k} t\right) \widetilde{d} x^{k}\right] \text { and } \\
\mathrm{d} \varphi=\frac{\partial \varphi}{\partial y^{4}}\left[\mathrm{~d} y^{4}+\left(\partial_{4} \varphi\right)^{-1}\left(\widetilde{\partial}_{k} \varphi\right) \widetilde{d} x^{k}\right]
\end{gathered}
$$

for $\widetilde{\partial}_{i} \varphi=\partial \varphi / \partial \widetilde{x}^{i}$ and $\partial_{a} \varphi=\partial \varphi / \partial y^{a}$. Choosing

$$
\begin{aligned}
& \underline{\circ}_{i}=\widetilde{\partial}_{i} n\left(x^{k}\right)=\left(\partial_{3} t\right)^{-1}\left(\widetilde{\partial}_{i} t\right) \text {, and } \\
& \underline{\stackrel{\circ}{\underline{\omega}}}_{i}=\widetilde{\partial}_{i} \Phi / \stackrel{\circ}{\Phi}^{*}=\left(\partial_{4} \varphi\right)^{-1}\left(\widetilde{\partial}_{i} \varphi\right),
\end{aligned}
$$

we express (97) as

$$
\begin{aligned}
& \mathrm{d} s^{2}=\underline{\stackrel{g}{g}}_{1^{\prime}}\left(\mathrm{d} \widetilde{x}^{1^{\prime}}\right)^{2}+\underline{\stackrel{g}{g}}_{2^{\prime}}\left(\widetilde{x}^{1^{\prime}}\right)\left(\mathrm{d} \widetilde{x}^{2^{\prime}}\right)^{2}+\underline{g}_{3}\left(x^{k}\left(\widetilde{x}^{k^{\prime}}\right)\right) \\
& \times\left[\mathrm{d} y^{3}+\underline{\circ}_{i}\left(\widetilde{x}^{k}\right) \mathrm{d} \widetilde{x}^{i}\right]^{2}+\underline{\circ}_{4}\left[\mathrm{~d} y^{4}+\underline{\stackrel{\circ}{*}}_{i}\left(\widetilde{x}^{k}\right) \mathrm{d} x^{i}\right]^{2}, \\
& \underline{g}_{4}\left(\widetilde{x}^{k}\left(\widetilde{x}^{k^{\prime}}\right)\right)=\left(\partial_{4} \varphi\right)^{2} r^{2}\left(\widetilde{x}^{\prime}\right) \sin \left(\widetilde{x}^{2^{\prime}}\right) \text { and } \underline{g}_{3}\left(\widetilde{x}^{k}\left(\widetilde{x}^{k^{\prime}}\right)\right) \\
& =-\left(\partial_{3} t\right)^{2}\left(1-\frac{M}{r}+\lambda r^{2}\right) \text {. }
\end{aligned}
$$

The prime d-metric (100) allows us to apply the AFDM and construct $\varepsilon$-deformations of geometric/physical objects and physical parameters as shown in Sect. 2.4.
For $\underline{g}_{3}=\underline{\circ}_{3}\left(\widetilde{x}^{1^{\prime}}\right)=\left(1-\frac{M}{r}+\lambda r^{2}\right)$ and $\left(\partial_{3} t\right)^{2}=1$ and anisotropically polarized mass $\tilde{M}(\varphi)=M\left[1+\varepsilon \cos \left(\omega_{0} \varphi+\right.\right.$ $\left.\varphi_{0}\right)$ ], we obtain

$$
\begin{aligned}
{ }^{s=0} h_{3} & =-\left(1-\frac{M}{r}+\lambda r^{2}\right)\left[1-\varepsilon \frac{M}{r} \frac{\cos \left(\omega_{0} \varphi+\varphi_{0}\right)}{1-\frac{M}{r}+\lambda r^{2}}\right] \\
& =\underline{h}_{3}\left(\widetilde{x}^{1^{\prime}}\right)\left[1-\varepsilon \frac{M}{r}\left(\underline{h}_{3}\right)^{-1} \cos \left(\omega_{0} \varphi+\varphi_{0}\right)\right] \\
& \simeq-\left[1-\frac{\tilde{M}(\varphi)}{r}+\lambda r^{2}\right] .
\end{aligned}
$$

The parametric equation of an ellipse with radial parameter $\stackrel{\circ}{+}_{+}=M$ and eccentricity $\varepsilon, r_{+} \simeq \frac{M}{1-\varepsilon \cos \left(\omega_{0} \varphi+\varphi_{0}\right)}$, can be determined in a simple way for $\lambda=0$. We have to find solutions of a third order algebraic equation in order to determine possible horizons for nontrivial $\lambda$.

We construct ellipsoidal deformations of d-metric (100) if $\chi={ }_{\varsigma} \chi=8 \frac{M}{r} \varsigma^{2} \stackrel{\circ}{\Phi}^{-2} \cos \left(\omega_{0} \varphi+\varphi_{0}\right)$, with identification (98). Following the same method as in the previous subsection but for ${ }_{5} \chi$ used for d-metric coefficients (83), we compute

$$
\begin{aligned}
& { }_{\varsigma} g_{i}=\underline{\stackrel{o}{g}}_{i}\left[1+\varepsilon \chi_{i}\right]=\left[1+\varepsilon e^{0} q 1{ }^{1} q / 2 \varsigma^{2}\right] \underline{g}_{i} \\
& \text { solution of 2-d Poisson equations (39); } \\
& \left.{ }_{\varsigma} h_{3}=\left[1+\varepsilon \quad{ }_{\varsigma} \chi_{3}\right]\right]_{3} \stackrel{\circ}{3}=\left[1-\varepsilon \frac{1}{8 \varsigma^{2} \underline{g}_{3}} \stackrel{\circ}{\Phi}^{2} \varsigma \chi\right] \underline{g}_{3} ; \\
& { }_{5} h_{4}=\left[1+\varepsilon{ }_{5} \chi_{3}\right] g_{3}^{\circ} \\
& =\left[1+\varepsilon\left(2\left({ }^{\varsigma} \chi+\frac{\stackrel{\circ}{\Phi}}{\Phi^{*}} \chi^{*}\right)+\frac{1}{8 \varsigma^{2} \underline{g}_{4}^{\circ}} \stackrel{\circ}{ }^{2} \varsigma \chi\right)\right] \underline{\stackrel{o}{g}}_{3} ; \\
& { }_{\varsigma} n_{i}=\left[1+\varepsilon{ }_{\varsigma}^{n} \chi_{i}\right] \stackrel{\circ}{n}_{i}=\left[1+\varepsilon \widetilde{n}_{i} \int \mathrm{d} y^{4}\left({ }^{\varsigma} \chi+\frac{\stackrel{\circ}{\Phi}}{\Phi^{*}}{ }^{\varsigma} \chi^{*}\right.\right. \\
& \left.\left.+\frac{5}{16 \varsigma^{2}} \frac{1}{\underline{g}_{4}}\left(\stackrel{\Phi}{ }^{2} \varsigma \chi\right)^{*}\right)\right] \underline{\stackrel{\circ}{n}}_{i}, \\
& { }_{5} w_{i}=\left[1+\varepsilon{ }_{5}^{w} \chi_{i}\right] \stackrel{\circ}{w}_{i} \\
& =\left[1+\varepsilon\left(\frac{\partial_{i}\left({ }^{5} \chi \stackrel{\circ}{\Phi}\right)}{\partial_{i} \stackrel{\circ}{\Phi}}-\frac{\left({ }^{5} \chi \stackrel{\circ}{\Phi}\right)^{*}}{\stackrel{\circ}{\Phi}^{*}}\right)\right] \underline{\stackrel{\leftrightarrow}{w}}_{i} ;
\end{aligned}
$$

where $\widetilde{n}_{i}\left(x^{k}\right)$ is a redefined integration function including contributions from the prime metric (100). The generating functions ${ }^{5} \chi$ and ${ }^{0} q$ can be determined for an ellipsoid configuration induced by the effective cosmological constant $\varsigma^{2}$ in $R^{2}$ gravity.

Finally, we generate a class of generic off-diagonal metrics for ellipsoid Kerr-de Sitter configurations related to the cosmological constant in heterotic string gravity,

$$
\begin{aligned}
\mathrm{d} s^{2}= & {\left[1+\varepsilon e^{0} q{ }^{1} q / 2 \varsigma^{2}\right]\left[\underline{g}_{1^{\prime}}\left(\mathrm{d} \widetilde{x}^{1^{\prime}}\right)^{2}+\underline{g}_{2^{\prime}}\left(\widetilde{x}^{1^{\prime}}\right)\left(\mathrm{d} \widetilde{x}^{2^{\prime}}\right)^{2}\right] } \\
& -\left[1-\varepsilon \frac{1}{8 \varsigma^{2} \underline{g}_{3}} \stackrel{\Phi}{\Phi}^{2} \varsigma \chi\right] \underline{g}_{3}\left(x^{k}\left(\widetilde{x}^{k^{\prime}}\right)\right)
\end{aligned}
$$




$$
\begin{aligned}
& \times\left[\mathrm{d} y^{3}+\left[1+\varepsilon \tilde{n}_{i} \int \mathrm{d} y^{4}\left({ }^{\varsigma} \chi+\frac{\stackrel{\circ}{\Phi}}{\Phi^{*}} \varsigma \chi^{*}\right.\right.\right. \\
& \left.\left.\left.+\frac{5}{16 \varsigma^{2}} \frac{1}{\underline{g}_{4}}\left(\stackrel{\Phi}{ }^{2} \varsigma \chi\right)^{*}\right)\right] \underline{\stackrel{\circ}{n}}_{i} \mathrm{~d} \tilde{x}^{i}\right]^{2} \\
& +\left[1+\varepsilon\left(2\left(\varsigma \chi+\frac{\stackrel{\circ}{\Phi}}{\Phi^{*}} \varsigma \chi^{*}\right)+\frac{1}{8 \varsigma^{2} \underline{g}_{4}} \stackrel{\circ}{ }^{2} \varsigma \chi\right)\right] \\
& \times \underline{\circ}_{4}\left[\mathrm{~d} \varphi+\left[1+\varepsilon\left(\frac{\partial_{i}\left({ }^{\varsigma} \chi \stackrel{\circ}{\Phi}\right)}{\partial_{i} \stackrel{\circ}{\Phi}}-\frac{\left({ }^{\varsigma} \chi \stackrel{\circ}{\Phi}^{*}\right.}{\Phi^{*}}\right)\right] \underline{\stackrel{\circ}{w}}_{i} \mathrm{~d} \widetilde{x}^{i}\right]^{2} \text {. }
\end{aligned}
$$

Such metrics have Killing symmetry on $\partial / \partial y^{3}$ and are completely defined by generating functions ${ }^{1} q$ and ${ }^{5} \chi$ and effective source $2 \varsigma^{2}={ }_{K} \Lambda$ induced from string theory. They define $\varepsilon$-deformations of Kerr-de Sitter black holes into ellipsoid configurations with effective (polarized) cosmological constants determined by constants in string theory and equivalent MGTs. If the LC-conditions are satisfied, such metrics can be modeled in GR with nontrivial cosmological constant.

\subsection{Extra-dimensional off-diagonal string modifications of the Kerr solutions}

Various classes of exact solutions in heterotic string gravity can be constructed, which depend on which type of effective sources (19) and (38) are chosen. As a result, various classes of generic off-diagonal deformations of the Kerr metric into higher-dimensional exact solutions can be constructed. In this subsection we shall construct and analyze a series of 6-d and 10-d solutions encoding possible higher-dimension interactions with effective cosmological constants, warping configurations, $f$-modifications and certain analogies to almostKä hler gravity models.

\subsubsection{6-d deformations with nontrivial cosmological constant}

Solutions are determined by certain configurations of the NSfields $\widehat{\mathbf{H}}_{\alpha_{1} \beta_{1} \mu_{1}}$ which are nontrivial on the first shell $s=1$, see Eqs. (21) and the respective term in (38),

$$
\begin{aligned}
& { }^{H} \Upsilon_{\mu_{1} v_{1}}=\frac{1}{4} \widehat{\mathbf{H}}_{\alpha_{1} \beta_{1} \mu_{1}} \widehat{\mathbf{H}}_{v_{1}}{ }^{\alpha_{1} \beta_{1}} \text { with effective constant }{ }^{H} \Lambda ; \\
& { }^{H} \Upsilon_{\mu_{1} v_{1}}=-\frac{6}{2}\left({ }^{H} s\right)^{2} \mathbf{g}_{\beta_{1} \mu_{1}}, \text { for }{ }^{H} \Lambda=-3\left({ }^{H} s\right)^{2},
\end{aligned}
$$

where the coefficient 3 is used for an effective 6-d space time with trivial extension on four other internal space coordinates. We introduce an effective source in the quadratic element (96) extended with one nontrivial extra 2 -d shell.
Such a family of generic off-diagonal stationary solutions is described by

$$
\begin{aligned}
& \mathrm{d} s_{K \eta \sigma d}^{2}=\left(1-\frac{e^{0} q{ }^{1} q}{12\left({ }^{H} s\right)^{2}}\right)\left[\left(\mathrm{d} x^{1^{\prime}}\right)^{2}+\left(\mathrm{d} x^{2^{\prime}}\right)^{2}\right]+\bar{A}[1 \\
& \left.-2 \varepsilon \tilde{\zeta} \sin \left(\omega_{0} \varphi+\varphi_{0}\right)\right]\left[\mathrm{d} y^{3^{\prime}}+\left(\varepsilon \partial_{k^{\prime}} \chi_{n\left(x^{i^{\prime}}\right)}\right.\right. \\
& \left.\left.-\partial_{k^{\prime}}\left(\widehat{y}^{3^{\prime}}+\varphi \frac{\bar{B}}{\bar{A}}\right)\right) \mathrm{d} x^{k^{\prime}}\right]^{2} \\
& +\left[1+\varepsilon\left(-\frac{\left[-24 \bar{A}\left(H_{s}\right)^{2}+\stackrel{\circ}{\Phi}^{2}\right] \widetilde{\zeta}}{12\left(H_{s}\right)^{2} \stackrel{\circ}{ }^{2}} \sin \left(\omega_{0} \varphi+\varphi_{0}\right)\right.\right. \\
& \left.\left.+\frac{16 \tilde{\zeta} \omega_{0} \bar{A}\left({ }^{H} s\right)^{2}}{\stackrel{\Phi}{ }^{2}} \cos \left(\omega_{0} \varphi+\varphi_{0}\right)\right)\right] \\
& \left.\times\left(\bar{C}-\frac{\bar{B}^{2}}{\bar{A}}\right)\left[\mathrm{d} \varphi+\varepsilon \partial_{i^{\prime}} \check{A}\right) \mathrm{d} x^{i^{\prime}}\right]^{2} \\
& +\left[1+\varepsilon \frac{1 \stackrel{\circ}{\Phi}^{2}{ }_{1}^{H} \chi}{12\left({ }^{H} s\right)^{2} \underline{\circ}_{5}}\right] \underline{\circ}_{5}\left(x^{k}\left(\widetilde{x}^{k^{\prime}}\right)\right) \\
& \times\left[\mathrm{d} y^{5}+\left[1+\varepsilon{ }^{1} \tilde{n}_{i_{1}} \int \mathrm{d} y^{6}\left({ }_{1}^{H} \chi+\frac{1 \stackrel{\circ}{\Phi}}{1 \Phi^{*}{ }_{1}}{ }_{1}^{H} \chi^{* 1}\right.\right.\right. \\
& \left.\left.\left.+\frac{5}{24\left(H^{H}\right)^{2} \underline{g}_{6}}\left({ }^{1} \stackrel{\circ}{\Phi}^{2}{ }_{1}^{H} \chi\right)^{* 1}\right)\right]{\stackrel{\circ}{i_{1}}}_{i_{1}} \mathrm{~d} \widetilde{x}^{i_{1}}\right]^{2} \\
& +\left[1+\varepsilon\left(2\left({ }_{1}^{H} \chi+\frac{1 \stackrel{\circ}{\Phi}}{1 \stackrel{\circ}{\Phi}^{* 1}}{ }_{1}^{H} \chi^{* 1}\right)+\frac{1 \stackrel{\circ}{\Phi}}{12\left({ }^{H} S\right)^{2} \underline{o}_{6}}{ }_{1}^{H} \chi\right)\right] \stackrel{\circ}{g}_{6}
\end{aligned}
$$

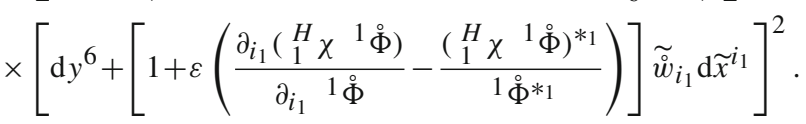

In the above formulas, we consider a generating function ${ }_{1}^{H} \chi\left(\tilde{x}^{i^{\prime}}, y^{4}, y^{6}\right)$, which results, in general, in nontrivial nonholonomic torsions encoding contributions of $\widehat{\mathbf{H}}_{\alpha_{1} \beta_{1} \mu_{1}}$ via an effective cosmological constant $\left({ }^{H} S\right)^{2}$. One considers

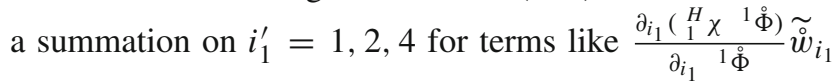
$\mathrm{d} \widetilde{x}^{i_{1}}$.

Off-diagonal extra-dimensional gravitational interactions modify a Kerr metric for any nontrivial cosmological constant determined by a corresponding ansatz for $\widehat{\mathbf{H}}_{\alpha_{1} \beta_{1} \mu_{1}}$ in 6 -d. In a similar form we can generalize the constructions in 8-d and 10-d with nontrivial extra shell components $\widehat{\mathbf{H}}_{\alpha_{s}} \beta_{s} \mu_{s}$ and ${ }^{R} \Lambda=0$ in (19).

\subsubsection{0-d deformations with NS 3-form and 6-d almost Kähler internal spaces}

The class of solutions (103) can be generalized for $10-\mathrm{d}$ spacetimes with indices of fields running over 10 -d values and nontrivial sources (34) redefined in (38), with nontriv- 
ial ${ }^{H} \Lambda=-5\left({ }^{H} s\right)^{2},{ }^{F} \Lambda=-5 n_{F}\left({ }^{F} s\right)^{2}$ and ${ }^{R} \Lambda=$ $-5 \operatorname{trn}_{R}\left({ }^{R} S\right)^{2}$ in $\Lambda={ }^{H} \Lambda+{ }^{F} \Lambda+{ }^{R} \Lambda$. The corresponding quadratic line element is

$$
\begin{aligned}
& \mathrm{d} s_{K \eta 10 d}^{2}=\left(1+e^{0} q \frac{{ }^{1} q}{4 \Lambda}\right)\left[\left(\mathrm{d} x^{1^{\prime}}\right)^{2}+\left(\mathrm{d} x^{2^{\prime}}\right)^{2}\right] \\
& +\bar{A}\left[1-2 \varepsilon \tilde{\zeta} \sin \left(\omega_{0} \varphi+\varphi_{0}\right)\right] \\
& \times\left[\mathrm{d} y^{3^{\prime}}+\left(\varepsilon \partial_{k^{\prime}} \times n\left(x^{i^{\prime}}\right)-\partial_{k^{\prime}}\left(\widehat{y}^{3^{\prime}}+\varphi \frac{\bar{B}}{\bar{A}}\right)\right) \mathrm{d} x^{k^{\prime}}\right]^{2} \\
& +\left[1+\varepsilon\left(\frac{\left[8 \bar{A} \Lambda+\AA^{2}\right] \tilde{\zeta}}{4 \Lambda \Phi^{2}} \sin \left(\omega_{0} \varphi+\varphi_{0}\right)\right.\right. \\
& \left.\left.-\frac{4 \widetilde{\zeta} \omega_{0} \bar{A} \Lambda}{\check{\Phi}^{2}} \cos \left(\omega_{0} \varphi+\varphi_{0}\right)\right)\right] \\
& \times\left(\bar{C}-\frac{\bar{B}^{2}}{\bar{A}}\right)\left[\mathrm{d} \varphi+\varepsilon\left(\partial_{i^{\prime}} \check{A}\right) \mathrm{d} x^{i^{\prime}}\right]^{2} \\
& +\left[1-\varepsilon \frac{1}{4 \Lambda \underline{g}_{5}^{\circ}}{ }^{1} \stackrel{\circ}{\Phi}^{2}{ }_{1}^{\Lambda} \chi\right] \underline{g}_{5}\left(x^{k}\left(\widetilde{x}^{k^{\prime}}\right)\right) \\
& \times\left[\mathrm{d} y^{5}+\left[1+\varepsilon{ }^{1} \widetilde{n}_{i_{1}} \int \mathrm{d} y^{6}\left({ }_{1}^{\Lambda} \chi+\frac{1{ }^{\circ}}{1 \Phi^{* 1}}{ }_{1}^{\Lambda} \chi^{*_{1}}\right.\right.\right. \\
& \left.\left.\left.-\frac{5}{8 \Lambda} \frac{1}{\underline{g}_{6}}\left({ }^{1} \stackrel{\Phi}{\Phi}^{2}{ }_{1}^{\Lambda} \chi\right)^{*_{1}}\right)\right] \underline{\circ}_{i_{1}} \mathrm{~d}^{\tilde{x}_{1}}\right]^{2} \\
& +\left[1+\varepsilon\left(2\left({ }_{1}^{\Lambda} \chi+\frac{1 \Phi}{1{ }^{*}{ }^{* 1}}{ }_{1}^{\Lambda} \chi^{* 1}\right)-\frac{1 \stackrel{\circ}{\Phi}^{\circ}}{4 \Lambda \underline{g}_{6}}{ }_{1} \chi\right)\right] \underline{g}_{6} \\
& \times\left[\mathrm{d} y^{6}+\left[1+\varepsilon\left(\frac{\partial_{i_{1}}\left(\begin{array}{l}
\Lambda \\
1
\end{array} \chi^{1} \Phi\right)}{\partial_{i_{1}} 1^{\circ} \Phi}-\frac{\left(\begin{array}{l}
\Lambda \\
1
\end{array} \chi^{1} \Phi^{\Phi^{*}}\right)^{* 1}}{1 \Phi^{* 1}}\right)\right] \widetilde{\tilde{w}}_{i_{1}} \mathrm{~d} \widetilde{x}^{i_{1}}\right]^{2} \\
& +\left[1-\varepsilon \frac{1}{4 \Lambda \underline{g}_{7}^{\circ}}{ }^{2} \stackrel{\circ}{\Phi}^{2}{ }_{2}^{\Lambda} \chi\right] \underline{g}_{7}\left(x^{k}\left(\widetilde{x}^{k^{\prime}}\right)\right) \\
& \times\left[\mathrm{d} y^{7}+\left[1+\varepsilon^{2} \widetilde{n}_{i_{2}} \int \mathrm{d} y^{8}\left({ }_{2}^{\Lambda} \chi+\frac{2{ }^{\circ}}{{ }^{2} \Phi^{* 2}}{ }_{2}^{\Lambda} \chi^{* 2}\right.\right.\right. \\
& \left.\left.\left.-\frac{5}{8 \Lambda} \frac{1}{\underline{g}_{8}}\left({ }^{2} \stackrel{\Phi}{ }^{2}{ }_{2}^{\Lambda} \chi\right)^{* 2}\right)\right] \underline{\stackrel{\circ}{n}}_{i_{2}} \mathrm{~d} \widetilde{x}^{i_{2}}\right]^{2} \\
& +\left[1+\varepsilon\left(2\left({ }_{2}^{\Lambda} \chi+\frac{2 \stackrel{\circ}{\Phi}}{2 \stackrel{\circ}{\Phi}_{2}}{ }_{2}^{\Lambda} \chi^{*_{2}}\right)-\frac{2 \stackrel{\circ}{\Phi}}{4 \Lambda \underline{g}_{8}^{\circ}}{ }_{2}^{\Lambda} \chi\right)\right] \stackrel{\circ}{g}_{8}
\end{aligned}
$$

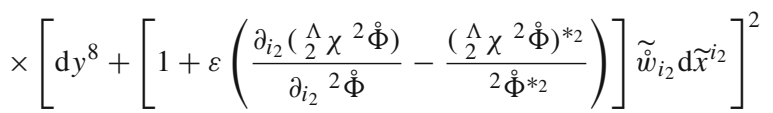

$$
\begin{aligned}
& +\left[1-\varepsilon \frac{1}{4 \Lambda \underline{g}_{9}^{\circ}} 3{ }^{2}{ }^{2}{ }_{3}^{\Lambda} \chi\right] \underline{g}_{9}\left(x^{k}\left(\widetilde{x}^{k^{\prime}}\right)\right)\left[\mathrm{d} y^{9}+\left[1+\varepsilon{ }^{3} \widetilde{n}_{i_{3}}\right.\right.
\end{aligned}
$$

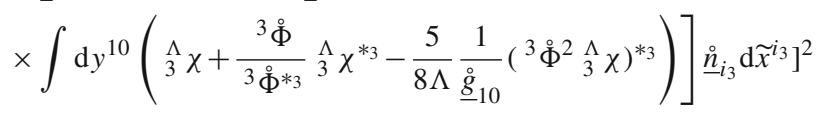

$$
\begin{aligned}
& +\left[1+\varepsilon\left(2\left(\frac{\Lambda}{3} \chi+\frac{3 \stackrel{\circ}{\Phi}}{3 \dot{\Phi}^{* 3}}{ }_{3}^{\Lambda} \chi^{* 3}\right)-\frac{3 \stackrel{8}{\Phi}}{4 \Lambda \underline{g}_{10}^{\circ}} \Lambda\right)\right]
\end{aligned}
$$

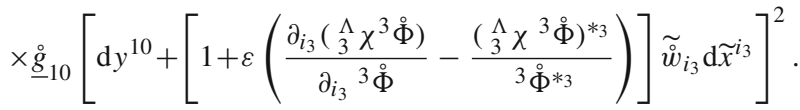

This generic off-diagonal stationary metric for 10-d spacetimes defines a very general class of stationary solutions of the nonholonomic equations of motion in heterotic gravity (12)-(15) with effective scalar field encoded into the Nconnection structure. It is important to use "shell by shell" nonholonomic variables for the quadratic element (104). Only in such cases we can understand the nonlinear symmetries and classify the types of generating and integration functions corresponding to horizontal and higher order vertical conventional sources. Such properties cannot be encoded in a minimal form if notations for indices running coordinate values from 0 to 9 are used as in standard papers on heterotic supergravity. For small $\varepsilon$-deformations, the 4 -d component of such metrics describes a Kerr type black ellipsoid with eccentricity $\varepsilon$ and $\varphi$-anisotropic polarization of physical constants and horizons. The nonholonomic deformations also encode sources from all gauge-like and interior space gravitational fields via a redefinition of generating functions.

Similar classes of non-vacuum solutions can also be modeled for Einstein-Finsler spaces if extra-dimensional coordinates are treated as velocity/momentum coordinates $[10,24,26,43,44,53,54]$. The metrics possess a respective Killing symmetry on $\partial_{t}$ and $\partial / \partial y^{9}$. Using the AFDM, we can construct solutions depending on all 10-d coordinates which may describe geometric evolution and time propagating Kerr black holes determined by heterotic string gravity effects. They define $\varepsilon$-deformations of Kerr-de Sitter black holes into ellipsoid configurations with effective cosmological constants determined by constants in GR, possible $f$ modifications and extra-dimension contributions [72]. By nonholonomic frame transforms and distortions of the canonical d-connection, the above metric can be rewritten in canonical almost-Kähler variables on 6-d internal space, as we prove in [48]. We omit such constructions (being very important in various models of deformation and brane quantization of MGTs and geometric evolution theories [10,11,63,70]) in this work.

\subsubsection{Off-diagonal solutions in standard 10-d heterotic string coordinates}

We can re-write the solution (104) in standard variables used in [1-7] with coordinates having prime Greek indices redefined in spherical 4-d coordinates (99) and extra dimensions,

$$
\begin{aligned}
& x^{\mu}=\left(x^{0}=x^{0^{\prime}}=t, x^{1}=x^{1^{\prime}}=r, x^{2}=x^{2^{\prime}}\right. \\
& \left.=\vartheta, x^{3}=x^{3^{\prime}}=\varphi, x^{4}=u^{5^{\prime}}=u^{5}, \ldots, x^{9}=v u^{10^{\prime}}=u^{10}\right),
\end{aligned}
$$

considering respective partial derivatives and explicit values for the functions $\bar{A}, \bar{B}, \bar{C}$ (87) determined by the 4-d Kerr black hole solution and explicit parameterizations of integration and generating functions, 
$\mathrm{d} s_{K \eta 10 d}^{2}=\left(1+e^{0} q(r) \frac{{ }^{1} q(r)}{4 \Lambda}\right)\left[(\mathrm{d} r)^{2}+\vartheta^{2}(\mathrm{~d} r)^{2}\right]$

$-\frac{r^{2}-2 m_{0}+a^{2}-a^{2} \sin ^{2} \vartheta}{r^{2}+a^{2} \cos ^{2} \vartheta}\left[1-2 \varepsilon \tilde{\zeta} \sin \left(\omega_{0} \varphi+\varphi_{0}\right)\right]$

$\times\left[\mathrm{d} t+\left(\varepsilon \partial_{r} x_{n}(r, \vartheta)-\partial_{r}\left[\widehat{y}^{3^{\prime}}(r, \vartheta, \varphi)+\varphi \frac{2 m_{0} a \sin ^{2} \vartheta}{\left(r^{2}-2 m_{0}+a^{2}-a^{2} \sin ^{2} \vartheta\right)}\right]\right) \mathrm{d} r\right.$

$\left.+\left(\varepsilon \partial_{\vartheta} x_{n}(r, \vartheta)-\partial_{\vartheta}\left[\widehat{y}^{3^{\prime}}(r, \vartheta, \varphi)+\varphi \frac{2 m_{0} a \sin ^{2} \vartheta}{\left(r^{2}-2 m_{0}+a^{2}-a^{2} \sin ^{2} \vartheta\right)}\right]\right) \mathrm{d} \vartheta\right]^{2}$

$+\left[1+\varepsilon\left(\frac{\left[\Phi^{2}(r, \vartheta, \varphi)-8 \bar{A} \Lambda \frac{r^{2}-2 m_{0}+a^{2}-a^{2} \sin ^{2} \vartheta}{r^{2}+a^{2} \cos ^{2} \vartheta}\right] \tilde{\zeta}}{4 \Lambda \AA^{2}(r, \vartheta, \varphi)} \sin \left(\omega_{0} \varphi+\varphi_{0}\right)\right.\right.$

$\left.\left.-\frac{4 \widetilde{\zeta} \omega_{0} \bar{A} \Lambda}{\check{\Phi}^{2}} \cos \left(\omega_{0} \varphi+\varphi_{0}\right)\right)\right]\left(\frac{\sin ^{2} \vartheta\left[\left(r^{2}+a^{2}\right)^{2}-\Delta a^{2} \sin ^{2} \vartheta\right]}{r^{2}+a^{2} \cos ^{2} \vartheta}\right.$

$\left.+\frac{4\left(m_{0}\right)^{2} a^{2} \sin ^{4} \vartheta}{\left(r^{2}+a^{2} \cos ^{2} \vartheta\right)\left(r^{2}-2 m_{0}+a^{2}-a^{2} \sin ^{2} \vartheta\right)}\right)$

$\times\left[\mathrm{d} \varphi+\varepsilon\left(\partial_{r} \check{A}(r, \vartheta, \varphi)\right) \mathrm{d} r+\varepsilon\left(\partial_{\vartheta} \check{A}(r, \vartheta, \varphi)\right) \mathrm{d} \vartheta\right]^{2}$

$+\left[1-\varepsilon \frac{1}{4 \Lambda \underline{g}_{5}^{\circ}(r, \vartheta, \varphi)}{ }^{1} \stackrel{\circ}{\Phi}^{2}\left(r, \vartheta, \varphi, x^{5}\right)_{1}^{\Lambda} \chi\left(r, \vartheta, \varphi, x^{5}\right)\right]$

$\times \underline{g}_{4}(r, \vartheta, \varphi)\left[\mathrm{d} x^{4}+\left[1+\varepsilon{ }^{1} \widetilde{n}_{1}(r, \vartheta, \varphi) \int \mathrm{d} x^{5}\left({ }_{1}^{\Lambda} \chi\left(r, \vartheta, \varphi, x^{5}\right)\right.\right.\right.$

$+\frac{1 \stackrel{\circ}{\Phi}\left(r, \vartheta, \varphi, x^{5}\right)}{\frac{\partial}{\partial x^{5}} 1 \stackrel{\circ}{\Phi}\left(r, \vartheta, \varphi, x^{5}\right)} \frac{\partial}{\partial x^{5}}{ }_{1}^{\Lambda} \chi\left(r, \vartheta, \varphi, x^{5}\right)$

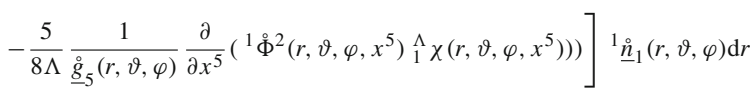

$+\varepsilon{ }^{1} \widetilde{n}_{2}(r, \vartheta, \varphi) \int \mathrm{d} x^{5}\left({ }_{1}^{\Lambda} \chi\left(r, \vartheta, \varphi, x^{5}\right)\right.$

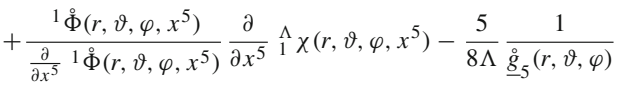

$\left.\left.\times \frac{\partial}{\partial x^{5}}\left({ }^{1} \Phi^{2}\left(r, \vartheta, \varphi, x^{5}\right){ }_{1}^{\Lambda} \chi\left(r, \vartheta, \varphi, x^{5}\right)\right)\right)\right]{ }^{1} \underline{\stackrel{\circ}{2}}_{2}(r, \vartheta, \varphi) \mathrm{d} \vartheta$

$+\varepsilon{ }^{1} \widetilde{n}_{3}(r, \vartheta, \varphi) \int \mathrm{d} x^{5}\left({ }_{1}^{\Lambda} \chi\left(r, \vartheta, \varphi, x^{5}\right)\right.$

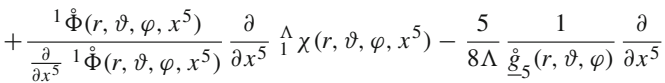

$\left.\left.\left.\times\left({ }^{1} \stackrel{\circ}{\Phi}^{2}\left(r, \vartheta, \varphi, x^{5}\right){ }_{1}^{\Lambda} \chi\left(r, \vartheta, \varphi, x^{5}\right)\right)\right)\right]{ }^{1} \underline{\underline{n}}_{3}(r, \vartheta, \varphi) \mathrm{d} \varphi\right]^{2}$

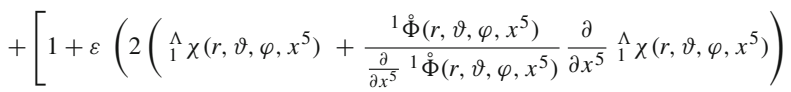

$\left.\left.-\frac{1 \stackrel{\Phi}{\Phi}\left(r, \vartheta, \varphi, x^{5}\right)}{4 \Lambda \underline{g}_{5}(r, \vartheta, \varphi)}{ }_{1}^{\Lambda} \chi\left(r, \vartheta, \varphi, x^{5}\right)\right)\right] \underline{\mathrm{g}}_{5}(r, \vartheta, \varphi)\left[\mathrm{d} x^{5}\right.$

$+\left[1+\varepsilon\left(\frac{\partial_{r}\left({ }_{1}^{\Lambda} \chi\left(r, \vartheta, \varphi, x^{5}\right){ }^{1} \stackrel{\oplus}{\Phi}\left(r, \vartheta, \varphi, x^{5}\right)\right)}{\partial_{r}{ }^{1} \stackrel{\oplus}{\Phi}\left(r, \vartheta, \varphi, x^{5}\right)}\right.\right.$

$\left.\left.-\frac{\frac{\partial}{\partial x^{5}}\left({ }_{1}^{\Lambda} \chi\left(r, \vartheta, \varphi, x^{5}\right){ }^{1} \stackrel{\oplus}{\Phi}\left(r, \vartheta, \varphi, x^{5}\right)\right)}{\frac{\partial}{\partial x^{5}}{ }^{1} \stackrel{\Phi}{\Phi}\left(r, \vartheta, \varphi, x^{5}\right)}\right)\right]{ }^{1} \widetilde{\tilde{w}}_{1}(r, \vartheta, \varphi) \mathrm{d} r$

$+\left[1+\varepsilon\left(\frac{\left.\partial_{\vartheta}\left(\begin{array}{l}\Lambda \\ 1\end{array}\right)\left(r, \vartheta, \varphi, x^{5}\right)^{1} \stackrel{\circ}{\Phi}\left(r, \vartheta, \varphi, x^{5}\right)\right)}{\partial_{\vartheta}{ }^{1} \Phi\left(r, \vartheta, \varphi, x^{5}\right)}\right.\right.$

$\left.\left.-\frac{\frac{\partial}{\partial x^{5}}\left({ }_{1}^{\Lambda} \chi\left(r, \vartheta, \varphi, x^{5}\right)^{1}{ }^{1} \Phi\left(r, \vartheta, \varphi, x^{5}\right)\right)}{\frac{\partial}{\partial x^{5}}{ }^{1} \Phi\left(r, \vartheta, \varphi, x^{5}\right)}\right)\right]{ }^{1} \widetilde{\widetilde{w}}_{2}(r, \vartheta, \varphi) \mathrm{d} \vartheta$

$+\left[1+\varepsilon\left(\frac{\partial_{\varphi}\left(\begin{array}{l}\Lambda \\ 1\end{array}\left(r, \vartheta, \varphi, x^{5}\right)^{1} \stackrel{\leftrightarrow}{\Phi}\left(r, \vartheta, \varphi, x^{5}\right)\right)}{\partial_{\varphi}{ }^{1} \stackrel{\circ}{\Phi}\left(r, \vartheta, \varphi, x^{5}\right)}\right.\right.$

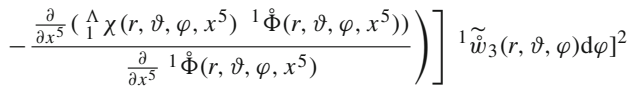

$+\left[1-\varepsilon \frac{1}{4 \Lambda \underline{g}_{6}\left(r, \vartheta, \varphi, x^{5}\right)}{ }^{2} \stackrel{\circ}{ }^{2}\left(r, \vartheta, \varphi, x^{5}, x^{7}\right) \stackrel{\Lambda}{2} \chi\left(r, \vartheta, \varphi, x^{5}, x^{7}\right)\right]$

$\times \underline{g}_{6}\left(r, \vartheta, \varphi, x^{5}\right)$ $\times\left[\mathrm{d} x^{6}+\left[1+\varepsilon{ }^{2} \widetilde{n}_{r}\left(r, \vartheta, \varphi, x^{5}\right) \int \mathrm{d} x^{7}\left({ }_{2}^{\Lambda} \chi\left(r, \vartheta, \varphi, x^{5}, x^{7}\right)\right.\right.\right.$

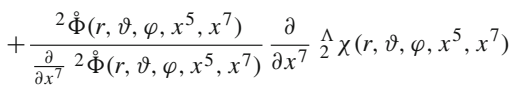

$-\frac{5}{8 \Lambda} \frac{1}{\underline{g}_{7}\left(r, \vartheta, \varphi, x^{5}\right)} \frac{\partial}{\partial x^{7}}\left({ }^{2} \stackrel{\circ}{\Phi}^{2}\left(r, \vartheta, \varphi, x^{5}, x^{7}\right) \stackrel{\Lambda}{2} \chi\right.$

$\left.\left.\left.\times\left(r, \vartheta, \varphi, x^{5}, x^{7}\right)\right)\right)\right]{ }^{2} \underline{\circ}_{r}\left(r, \vartheta, \varphi, x^{5}\right) \mathrm{d} r$

$+\left[1+\varepsilon{ }^{2} \widetilde{n}_{\vartheta}\left(r, \vartheta, \varphi, x^{5}\right) \int \mathrm{d} x^{7}\left({ }_{2}^{\Lambda} \chi\left(r, \vartheta, \varphi, x^{5}, x^{7}\right)\right.\right.$

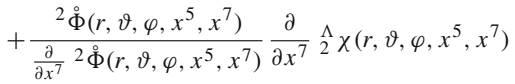

$\left.\left.-\frac{5}{8 \Lambda} \frac{1}{\underline{g}_{7}\left(r, \vartheta, \varphi, x^{5}\right)} \frac{\partial}{\partial x^{7}}\left({ }^{2} \stackrel{\Phi}{\Phi}^{2}\left(r, \vartheta, \varphi, x^{5}, x^{7}\right) \stackrel{\Lambda}{2} \chi\left(r, \vartheta, \varphi, x^{5}, x^{7}\right)\right)\right)\right]$

$\times \stackrel{\circ}{\underline{n}}_{\vartheta}\left(r, \vartheta, \varphi, x^{5}\right) \mathrm{d} \vartheta$

$+\left[1+\varepsilon{ }^{2} \widetilde{n}_{\varphi}\left(r, \vartheta, \varphi, x^{5}\right) \int \mathrm{d} x^{7}\left(\frac{\Lambda}{2} \chi\left(r, \vartheta, \varphi, x^{5}, x^{7}\right)\right.\right.$

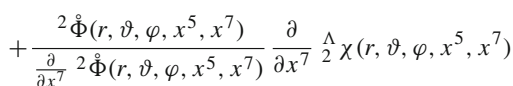

$\left.\left.-\frac{5}{8 \Lambda} \frac{1}{\underline{g}_{7}\left(r, \vartheta, \varphi, x^{5}\right)} \frac{\partial}{\partial x^{7}}\left({ }^{2} \dot{\Phi}^{2}\left(r, \vartheta, \varphi, x^{5}, x^{7}\right) \stackrel{\Lambda}{2} \chi\left(r, \vartheta, \varphi, x^{5}, x^{7}\right)\right)\right)\right]$

$\times \stackrel{\circ}{\underline{n}}_{\varphi}\left(r, \vartheta, \varphi, x^{5}\right) \mathrm{d} \varphi$

$+\left[1+\varepsilon{ }^{2} \widetilde{n}_{5}\left(r, \vartheta, \varphi, x^{5}\right) \int \mathrm{d} x^{7}\left(\frac{\Lambda}{2} \chi\left(r, \vartheta, \varphi, x^{5}, x^{7}\right)\right.\right.$

$+\frac{2 \stackrel{\circ}{\Phi}\left(r, \vartheta, \varphi, x^{5}, x^{7}\right)}{\frac{\partial}{\partial x^{7}} 2 \stackrel{\circ}{\Phi}\left(r, \vartheta, \varphi, x^{5}, x^{7}\right)} \frac{\partial}{\partial x^{7}} \stackrel{\Lambda}{2} \chi\left(r, \vartheta, \varphi, x^{5}, x^{7}\right)$

$\left.\left.-\frac{5}{8 \Lambda} \frac{1}{\underline{g}_{7}^{\circ}\left(r, \vartheta, \varphi, x^{5}\right)} \frac{\partial}{\partial x^{7}}\left({ }^{2} \stackrel{\circ}{\Phi}^{2}\left(r, \vartheta, \varphi, x^{5}, x^{7}\right) \stackrel{\Lambda}{2} \chi\left(r, \vartheta, \varphi, x^{5}, x^{7}\right)\right)\right)\right]$

$\left.\times{ }^{2} \underline{\circ}_{5}\left(r, \vartheta, \varphi, x^{5}\right) \mathrm{d} x^{5}\right]^{2}$

$+\left[1+\varepsilon\left(2\left({ }_{2}^{\Lambda} \chi\left(r, \vartheta, \varphi, x^{5}, x^{7}\right)+\frac{2 \stackrel{\circ}{\Phi}\left(r, \vartheta, \varphi, x^{5}, x^{7}\right)}{{\frac{\partial}{\partial x^{7}}}^{2} \dot{\Phi}\left(r, \vartheta, \varphi, x^{5}, x^{7}\right)}\right.\right.\right.$

$\left.\times \frac{\partial}{\partial x^{7}} \stackrel{\Lambda}{2} \chi\left(r, \vartheta, \varphi, x^{5}, x^{7}\right)\right)$

$\left.\left.-\frac{2 \stackrel{\circ}{\Phi}\left(r, \vartheta, \varphi, x^{5}, x^{7}\right)}{4 \Lambda \underline{g}_{7}^{\circ}\left(r, \vartheta, \varphi, x^{5}, x^{7}\right)} \Lambda \chi \gamma\left(r, \vartheta, \varphi, x^{5}, x^{7}\right)\right)\right] \underline{g}_{7}\left(r, \vartheta, \varphi, x^{5}\right)$

$\times\left[\mathrm{d} x^{7}+\left[1+\varepsilon\left(\frac{\partial_{r}\left(\frac{\Lambda}{2} \chi\left(r, \vartheta, \varphi, x^{5}, x^{7}\right)^{2} \Phi\left(r, \vartheta, \varphi, x^{5}, x^{7}\right)\right)}{\partial_{r}{ }^{2} \Phi\left(r, \vartheta, \varphi, x^{5}, x^{7}\right)}\right.\right.\right.$

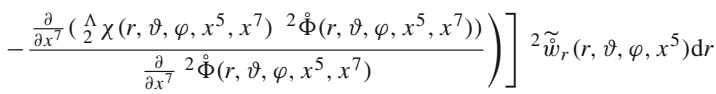

$+\left[1+\varepsilon\left(\frac{\partial_{\vartheta}\left({ }_{2}^{\Lambda} \chi\left(r, \vartheta, \varphi, x^{5}, x^{7}\right){ }^{2} \stackrel{\leftrightarrow}{\Phi}\left(r, \vartheta, \varphi, x^{5}, x^{7}\right)\right)}{\partial_{\vartheta}{ }^{2} \stackrel{\leftrightarrow}{\Phi}\left(r, \vartheta, \varphi, x^{5}, x^{7}\right)}\right.\right.$

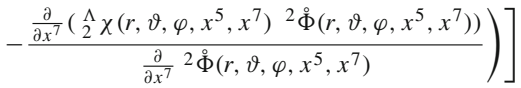

$\times{ }^{2} \widetilde{\tilde{w}}_{\vartheta}\left(r, \vartheta, \varphi, x^{5}\right) \mathrm{d} \vartheta$

$+\left[1+\varepsilon\left(\frac{\partial_{\varphi}\left(\frac{\Lambda}{2} \chi\left(r, \vartheta, \varphi, x^{5}, x^{7}\right)^{2} \stackrel{\circ}{\Phi}\left(r, \vartheta, \varphi, x^{5}, x^{7}\right)\right)}{\partial_{\varphi}{ }^{2} \stackrel{\circ}{\Phi}\left(r, \vartheta, \varphi, x^{5}, x^{7}\right)}\right.\right.$

$\left.\left.-\frac{\frac{\partial}{\partial x^{7}}\left({ }_{2}^{\Lambda} \chi\left(r, \vartheta, \varphi, x^{5}, x^{7}\right)^{2} \stackrel{\leftrightarrow}{\Phi}\left(r, \vartheta, \varphi, x^{5}, x^{7}\right)\right)}{\frac{\partial}{\partial x^{7}}{ }^{2} \Phi\left(r, \vartheta, \varphi, x^{5}, x^{7}\right)}\right)\right]$

$\times{ }^{2} \widetilde{\tilde{w}}_{\varphi}\left(r, \vartheta, \varphi, x^{5}\right) \mathrm{d} \varphi$

$+\left[1+\varepsilon\left(\frac{\frac{\partial}{\partial x^{5}}\left({ }_{2}^{\Lambda} \chi\left(r, \vartheta, \varphi, x^{5}, x^{7}\right){ }^{2} \stackrel{\circ}{\Phi}\left(r, \vartheta, \varphi, x^{5}, x^{7}\right)\right)}{\frac{\partial}{\partial x^{5}}{ }^{2} \stackrel{\circ}{\Phi}\left(r, \vartheta, \varphi, x^{5}, x^{7}\right)}\right.\right.$

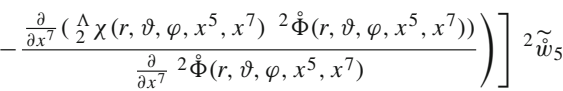




$$
\begin{aligned}
& \left.\times\left(r, \vartheta, \varphi, x^{5}\right) \mathrm{d} x^{5}\right]^{2}+\left[1-\varepsilon \frac{1}{4 \Lambda \underline{g}_{8}\left(r, \vartheta, \varphi, x^{5}, x^{6}, x^{7}\right)}{ }^{3} \AA^{2}\right. \\
& \left.\times\left(r, \vartheta, \varphi, x^{5}, x^{6}, x^{7}, x^{9}\right){ }_{3}^{\Lambda} \chi\left(r, \vartheta, \varphi, x^{5}, x^{6}, x^{7}, x^{9}\right)\right] \underline{g}_{8}\left(r, \vartheta, \varphi, x^{5}, x^{6}, x^{7}\right) \\
& \times\left[\mathrm{d} x^{8}+\left[1+\varepsilon{ }^{3} \widetilde{n}_{r}\left(r, \vartheta, \varphi, x^{5}, x^{6}, x^{7}\right) \int \mathrm{d} x^{9}\right.\right.
\end{aligned}
$$

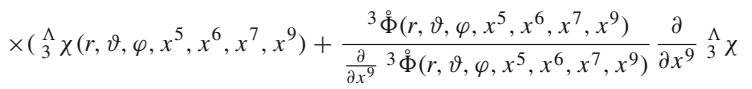

$$
\begin{aligned}
& \times\left(r, \vartheta, \varphi, x^{5}, x^{6}, x^{7}, x^{9}\right)-\frac{5}{8 \Lambda} \frac{1}{\underline{g}_{9}\left(r, \vartheta, \varphi, x^{5}, x^{6}, x^{7}\right)} \frac{\partial}{\partial x^{9}} \\
& \times\left({ }^{3} \AA^{2}\left(r, \vartheta, \varphi, x^{5}, x^{6}, x^{7}, x^{9}\right){ }_{3}^{\Lambda} \chi\right. \\
& \left.\left.\left.\times\left(r, \vartheta, \varphi, x^{5}, x^{6}, x^{7}, x^{9}\right)\right)\right)\right]{ }^{3} \underline{n}_{r}\left(r, \vartheta, \varphi, x^{5}, x^{6}, x^{7}\right) \mathrm{d} r \\
& +\left[1+\varepsilon{ }^{3} \widetilde{n}_{\vartheta}\left(r, \vartheta, \varphi, x^{5}, x^{6}, x^{7}\right) \int \mathrm{d} x^{9}\left({ }_{3}^{\Lambda} \chi\left(r, \vartheta, \varphi, x^{5}, x^{6}, x^{7}, x^{9}\right)\right.\right. \\
& +\frac{3 \stackrel{\circ}{\Phi}\left(r, \vartheta, \varphi, x^{5}, x^{6}, x^{7}, x^{9}\right)}{{\frac{\partial}{\partial x^{9}}}^{3} \stackrel{\circ}{\Phi}\left(r, \vartheta, \varphi, x^{5}, x^{6}, x^{7}, x^{9}\right)} \frac{\partial}{\partial x^{9}}{ }_{3}^{\Lambda} \chi\left(r, \vartheta, \varphi, x^{5}, x^{6}, x^{7}, x^{9}\right) \\
& -\frac{5}{8 \Lambda} \frac{1}{\underline{g}_{9}\left(r, \vartheta, \varphi, x^{5}, x^{6}, x^{7}\right)} \frac{\partial}{\partial x^{9}} \\
& \left.\left.\times\left({ }^{3} \stackrel{\circ}{\Phi}^{2}\left(r, \vartheta, \varphi, x^{5}, x^{6}, x^{7}, x^{9}\right){ }_{3}^{\Lambda} \chi\left(r, \vartheta, \varphi, x^{5}, x^{6}, x^{7}, x^{9}\right)\right)\right)\right] \\
& \times{ }^{3} \underline{\circ}_{\vartheta}\left(r, \vartheta, \varphi, x^{5}, x^{6}, x^{7}\right) \mathrm{d} \vartheta+\left[1+\varepsilon{ }^{3} \widetilde{n}_{\varphi}\left(r, \vartheta, \varphi, x^{5}, x^{6}, x^{7}\right)\right. \\
& \times \int \mathrm{d} x^{9}\left({ }_{3}^{\Lambda} \chi\left(r, \vartheta, \varphi, x^{5}, x^{6}, x^{7}, x^{9}\right)\right. \\
& +\frac{{ }^{3} \Phi\left(r, \vartheta, \varphi, x^{5}, x^{6}, x^{7}, x^{9}\right)}{\frac{\partial}{\partial x^{9}} \stackrel{3}{\Phi}\left(r, \vartheta, \varphi, x^{5}, x^{6}, x^{7}, x^{9}\right)} \frac{\partial}{\partial x^{9}} \stackrel{\Lambda}{3} \chi\left(r, \vartheta, \varphi, x^{5}, x^{6}, x^{7}, x^{9}\right) \\
& -\frac{5}{8 \Lambda} \frac{1}{\underline{g}_{9}\left(r, \vartheta, \varphi, x^{5}, x^{6}, x^{7}\right)} \frac{\partial}{\partial x^{9}}\left({ }^{3} \stackrel{\Phi}{ }^{2}\left(r, \vartheta, \varphi, x^{5}, x^{6}, x^{7}, x^{9}\right)_{3}^{\Lambda}\right.
\end{aligned}
$$

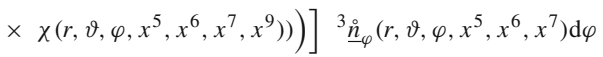

$$
\begin{aligned}
& +\left[1+\varepsilon{ }^{3} \widetilde{n}_{5}\left(r, \vartheta, \varphi, x^{5}, x^{6}, x^{7}\right) \int \mathrm{d} x^{9}\left({ }_{3}^{\Lambda} \chi\left(r, \vartheta, \varphi, x^{5}, x^{6}, x^{7}, x^{9}\right)\right.\right. \\
& +\frac{3 \stackrel{\circ}{\Phi}\left(r, \vartheta, \varphi, x^{5}, x^{6}, x^{7}, x^{9}\right)}{\frac{\partial}{\partial x^{9}}{ }^{3} \Phi\left(r, \vartheta, \varphi, x^{5}, x^{6}, x^{7}, x^{9}\right)} \frac{\partial}{\partial x^{9}}{ }_{3}^{\Lambda} \chi\left(r, \vartheta, \varphi, x^{5}, x^{6}, x^{7}, x^{9}\right) \\
& -\frac{5}{8 \Lambda} \frac{1}{\underline{g}_{9}\left(r, \vartheta, \varphi, x^{5}, x^{6}, x^{7}\right)} \frac{\partial}{\partial x^{9}}\left({ }^{3} \stackrel{\Phi}{ }^{2}\left(r, \vartheta, \varphi, x^{5}, x^{6}, x^{7}, x^{9}\right)_{3}^{\Lambda}\right. \\
& \left.\left.\left.\times \chi\left(r, \vartheta, \varphi, x^{5}, x^{6}, x^{7}, x^{9}\right)\right)\right)\right]{ }^{3} \underline{n}_{5}\left(r, \vartheta, \varphi, x^{5}, x^{6}, x^{7}\right) \mathrm{d} x^{5} \\
& +\left[1+\varepsilon{ }^{3} \widetilde{n}_{6}\left(r, \vartheta, \varphi, x^{5}, x^{6}, x^{7}\right) \int \mathrm{d} x^{9}\left({ }_{3}^{\Lambda} \chi\left(r, \vartheta, \varphi, x^{5}, x^{6}, x^{7}, x^{9}\right)\right.\right. \\
& +\frac{{ }^{3} \Phi\left(r, \vartheta, \varphi, x^{5}, x^{6}, x^{7}, x^{9}\right)}{\frac{\partial}{\partial x^{9}}{ }^{3} \Phi\left(r, \vartheta, \varphi, x^{5}, x^{6}, x^{7}, x^{9}\right)} \frac{\partial}{\partial x^{9}} \stackrel{\Lambda}{3} \chi\left(r, \vartheta, \varphi, x^{5}, x^{6}, x^{7}, x^{9}\right) \\
& -\frac{5}{8 \Lambda} \frac{1}{\underline{g}_{9}\left(r, \vartheta, \varphi, x^{5}, x^{6}, x^{7}\right)} \frac{\partial}{\partial x^{9}}\left({ }^{3} \stackrel{\Phi}{ }^{2}\left(r, \vartheta, \varphi, x^{5}, x^{6}, x^{7}, x^{9}\right)_{3}^{\Lambda}\right. \\
& \left.\left.\left.\times \chi\left(r, \vartheta, \varphi, x^{5}, x^{6}, x^{7}, x^{9}\right)\right)\right)\right]^{3}{\stackrel{3}{n_{6}}}_{6} \\
& \times\left(r, \vartheta, \varphi, x^{5}, x^{6}, x^{7}\right) \mathrm{d} x^{6}+\left[1+\varepsilon{ }^{3} \tilde{n}_{7}\left(r, \vartheta, \varphi, x^{5}, x^{6}, x^{7}\right)\right. \\
& \times \int \mathrm{d} x^{9}\left({ }_{3}^{\Lambda} \chi\left(r, \vartheta, \varphi, x^{5}, x^{6}, x^{7}, x^{9}\right)\right.
\end{aligned}
$$

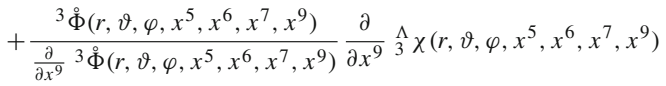

$$
\begin{aligned}
& -\frac{5}{8 \Lambda} \frac{1}{\underline{g}_{9}\left(r, \vartheta, \varphi, x^{5}, x^{6}, x^{7}\right)} \frac{\partial}{\partial x^{9}}\left({ }^{3} \dot{\Phi}^{2}\left(r, \vartheta, \varphi, x^{5}, x^{6}, x^{7}, x^{9}\right)_{3}^{\Lambda}\right. \\
& \left.\left.\left.\times \chi\left(r, \vartheta, \varphi, x^{5}, x^{6}, x^{7}, x^{9}\right)\right)\right)\right]{ }^{3}{\stackrel{\circ}{n_{7}}} \\
& \left.\times\left(r, \vartheta, \varphi, x^{5}, x^{6}, x^{7}\right) \mathrm{d} x^{7}\right]^{2}+[1+\varepsilon \\
& \times\left(2 \left({ }_{3}^{\Lambda} \chi\left(r, \vartheta, \varphi, x^{5}, x^{6}, x^{7}, x^{9}\right)+\frac{3 \stackrel{\leftrightarrow}{\Phi}\left(r, \vartheta, \varphi, x^{5}, x^{6}, x^{7}, x^{9}\right)}{\frac{\partial}{\partial x^{9}}{ }^{3} \Phi\left(r, \vartheta, \varphi, x^{5}, x^{6}, x^{7}, x^{9}\right)}\right.\right.
\end{aligned}
$$

$\left.\times \frac{\partial}{\partial x^{9}} \stackrel{\Lambda}{3} \chi\left(r, \vartheta, \varphi, x^{5}, x^{6}, x^{7}, x^{9}\right)\right)-\frac{3 \stackrel{\circ}{\Phi}\left(r, \vartheta, \varphi, x^{5}, x^{6}, x^{7}, x^{9}\right)}{4 \Lambda \underline{g}_{9}^{\circ}\left(r, \vartheta, \varphi, x^{5}, x^{6}, x^{7}, x^{9}\right)}{ }_{3}^{\Lambda}$

$\left.\left.\times \chi\left(r, \vartheta, \varphi, x^{5}, x^{6}, x^{7}, x^{9}\right)\right)\right] \underline{g}_{9}\left(r, \vartheta, \varphi, x^{5}, x^{6}, x^{7}\right)$

$\times\left[\mathrm{d} x^{9}+\left[1+\varepsilon\left(\frac{\partial_{r}\left(\begin{array}{l}\Lambda \\ 3\end{array}\left(r, \vartheta, \varphi, x^{5}, x^{6}, x^{7}, x^{9}\right){ }^{3} \stackrel{\circ}{\Phi}\left(r, \vartheta, \varphi, x^{5}, x^{6}, x^{7}, x^{9}\right)\right)}{\partial_{i_{3}}{ }^{3} \stackrel{\circ}{\Phi}\left(r, \vartheta, \varphi, x^{5}, x^{6}, x^{7}, x^{9}\right)}\right.\right.\right.$

$\left.\left.-\frac{\frac{\partial}{\partial x^{9}}\left({ }_{3}^{\Lambda} \chi\left(r, \vartheta, \varphi, x^{5}, x^{6}, x^{7}, x^{9}\right)^{3}{ }^{3} \Phi\left(r, \vartheta, \varphi, x^{5}, x^{6}, x^{7}, x^{9}\right)\right)}{\frac{\partial}{\partial x^{9}}{ }^{3} \Phi\left(r, \vartheta, \varphi, x^{5}, x^{6}, x^{7}, x^{9}\right)}\right)\right] 3$

$\times \widetilde{\tilde{w}}_{r}\left(r, \vartheta, \varphi, x^{5}, x^{6}, x^{7}\right) \mathrm{d} r$

$+\left[1+\varepsilon\left(\frac{\partial_{r}\left({ }_{3}^{\Lambda} \chi\left(r, \vartheta, \varphi, x^{5}, x^{6}, x^{7}, x^{9}\right){ }^{3} \Phi\left(r, \vartheta, \varphi, x^{5}, x^{6}, x^{7}, x^{9}\right)\right)}{\partial_{r}{ }^{3} \stackrel{\leftrightarrow}{\Phi}\left(r, \vartheta, \varphi, x^{5}, x^{6}, x^{7}, x^{9}\right)}\right.\right.$

$\left.\left.-\frac{\frac{\partial}{\partial x^{9}}\left({ }_{3}^{\Lambda} \chi\left(r, \vartheta, \varphi, x^{5}, x^{6}, x^{7}, x^{9}\right)^{3}{ }^{3} \Phi\left(r, \vartheta, \varphi, x^{5}, x^{6}, x^{7}, x^{9}\right)\right)}{{\frac{\partial}{\partial x^{9}}}^{3}{ }^{3}\left(r, \vartheta, \varphi, x^{5}, x^{6}, x^{7}, x^{9}\right)}\right)\right] 3$

$\times \widetilde{w}_{r}\left(r, \vartheta, \varphi, x^{5}, x^{6}, x^{7}\right) \mathrm{d} r$

$+\left[1+\varepsilon\left(\frac{\partial_{\vartheta}\left({ }_{3}^{\Lambda} \chi\left(r, \vartheta, \varphi, x^{5}, x^{6}, x^{7}, x^{9}\right)^{3} \stackrel{\circ}{\Phi}\left(r, \vartheta, \varphi, x^{5}, x^{6}, x^{7}, x^{9}\right)\right)}{\partial_{\vartheta}{ }^{3} \stackrel{\circ}{\Phi}\left(r, \vartheta, \varphi, x^{5}, x^{6}, x^{7}, x^{9}\right)}\right.\right.$

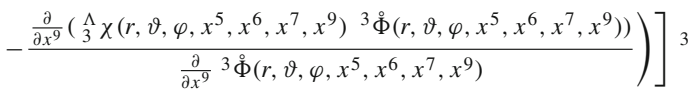

$\times \widetilde{\tilde{w}}_{\vartheta}\left(r, \vartheta, \varphi, x^{5}, x^{6}, x^{7}\right) \mathrm{d} \vartheta$

$+\left[1+\varepsilon\left(\frac{\partial_{\varphi}\left({ }_{3}^{\Lambda} \chi\left(r, \vartheta, \varphi, x^{5}, x^{6}, x^{7}, x^{9}\right){ }^{3} \stackrel{\circ}{\Phi}\left(r, \vartheta, \varphi, x^{5}, x^{6}, x^{7}, x^{9}\right)\right)}{\partial_{\varphi}{ }^{3} \stackrel{\circ}{\Phi}\left(r, \vartheta, \varphi, x^{5}, x^{6}, x^{7}, x^{9}\right)}\right.\right.$

$\left.\left.-\frac{\frac{\partial}{\partial x^{9}}\left({ }_{3}^{\Lambda} \chi\left(r, \vartheta, \varphi, x^{5}, x^{6}, x^{7}, x^{9}\right)^{3}{ }^{3} \Phi\left(r, \vartheta, \varphi, x^{5}, x^{6}, x^{7}, x^{9}\right)\right)}{{\frac{\partial}{\partial x^{9}}}^{3}{ }^{\circ}\left(r, \vartheta, \varphi, x^{5}, x^{6}, x^{7}, x^{9}\right)}\right)\right] 3$

$\times \widetilde{\tilde{w}}_{\varphi}\left(r, \vartheta, \varphi, x^{5}, x^{6}, x^{7}\right) \mathrm{d} \varphi$

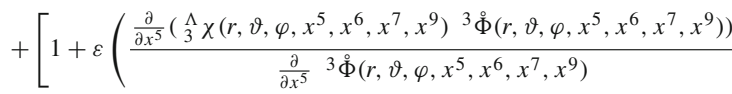

$\left.\left.-\frac{\frac{\partial}{\partial x^{9}}\left({ }_{3}^{\Lambda} \chi\left(r, \vartheta, \varphi, x^{5}, x^{6}, x^{7}, x^{9}\right)^{3}{ }^{3} \Phi\left(r, \vartheta, \varphi, x^{5}, x^{6}, x^{7}, x^{9}\right)\right)}{\frac{\partial}{\partial x^{9}}{ }^{3} \Phi\left(r, \vartheta, \varphi, x^{5}, x^{6}, x^{7}, x^{9}\right)}\right)\right] 3$

$\times \widetilde{\tilde{w}}_{5}\left(r, \vartheta, \varphi, x^{5}, x^{6}, x^{7}\right) \mathrm{d} x^{5}$

$+\left[1+\varepsilon\left(\frac{\frac{\partial}{\partial x^{6}}\left({ }_{3}^{\Lambda} \chi\left(r, \vartheta, \varphi, x^{5}, x^{6}, x^{7}, x^{9}\right){ }^{3} \stackrel{\circ}{\Phi}\left(r, \vartheta, \varphi, x^{5}, x^{6}, x^{7}, x^{9}\right)\right)}{\frac{\partial}{\partial x^{6}}{ }^{3} \stackrel{\circ}{\Phi}\left(r, \vartheta, \varphi, x^{5}, x^{6}, x^{7}, x^{9}\right)}\right.\right.$

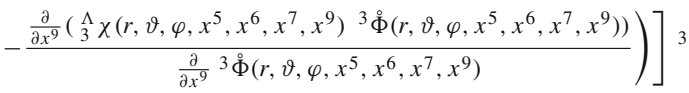

$\times \widetilde{\tilde{w}}_{6}\left(r, \vartheta, \varphi, x^{5}, x^{6}, x^{7}\right) \mathrm{d} x^{6}$

$+\left[1+\varepsilon\left(\frac{\frac{\partial}{\partial x^{7}}\left({ }_{3}^{\Lambda} \chi\left(r, \vartheta, \varphi, x^{5}, x^{6}, x^{7}, x^{9}\right){ }^{3} \stackrel{\oplus}{\Phi}\left(r, \vartheta, \varphi, x^{5}, x^{6}, x^{7}, x^{9}\right)\right)}{\frac{\partial}{\partial x^{7}}{ }^{3} \stackrel{\leftrightarrow}{\Phi}\left(r, \vartheta, \varphi, x^{5}, x^{6}, x^{7}, x^{9}\right)}\right.\right.$

$\left.\left.-\frac{\frac{\partial}{\partial x^{9}}\left({ }_{3}^{\Lambda} \chi\left(r, \vartheta, \varphi, x^{5}, x^{6}, x^{7}, x^{9}\right){ }^{3} \stackrel{\circ}{\Phi}\left(r, \vartheta, \varphi, x^{5}, x^{6}, x^{7}, x^{9}\right)\right)}{{\frac{\partial}{\partial x^{9}}}^{3}{ }^{\circ}\left(r, \vartheta, \varphi, x^{5}, x^{6}, x^{7}, x^{9}\right)}\right)\right]$

$\left.\times{ }^{3} \widetilde{\tilde{w}}_{7}\left(r, \vartheta, \varphi, x^{5}, x^{6}, x^{7}\right) \mathrm{d} x^{6}\right]^{2}$.

The above quadratic elements transform into a trivial embedding of the 4-d Kerr solution into a 10-d spacetime if $\varepsilon \rightarrow 0$ and primary data (labeled by circles) can be transformed into a diagonal 6-d internal space.

The solution (105) is equivalent to (104) up to a redefinition of the coordinates and some integration functions. It is difficult to see "shell by shell" nonlinear symmetries and construct generic off-diagonal solutions in the variant with standard variables used in heterotic string gravity. In former non $\mathrm{N}$-adapted variables, the formulas are much more cumbersome and less adapted for studying the limits to wellknown solutions and generalizations with nontrivial back- 
grounds and extra-dimensional contributions. To elaborate and apply a corresponding "shell by shell" $\mathrm{N}$-adapted geometric techniques of constructing exact solutions in $4 \mathrm{~d}$ and extra-dimension theories is important both from a mathematical stand point and a physical point of view.

\section{Outlook and concluding remarks}

In this work, we have applied the anholonomic frame method, AFDM, for constructing new classes of stationary solutions of motion equations in heterotic supergravity. Such solutions have generic off-diagonal metrics for effective ten-dimensional, 10-d, spacetimes enabled with generalized connections and depend on all possible 4-d and extradimensional space coordinates. They admit subclasses of solutions with warping on coordinate $y^{4}$, nearly almostKähler 6-d internal manifolds in the presence of nonholonomically deformed gravitational and gauge instantons. The almost-K ähler structure is necessary if we want to generate the Kerr metric with possible (off-) diagonal and nonholonomic deformations to black ellipsoid type solutions with locally anisotropic polarized physical constants, small deformations of horizons, embedding into nontrivial extradimension vacuum gravitational fields and/or gauge configurations. These solutions preserve two real supercharges corresponding to $N=1 / 2$ supersymmetry from the viewpoint of four non-compact dimensions and various nonholonomic deformations.

Following the AFDM, we can integrate the equations of motion in heterotic supergravity in very general forms with dependence on all 10-d spacetime coordinates. Such constructions are possible to more general classes of almostKähler nonholonomic variables and so-called canonical nonholonomic variables. This allows us to decouple string modified Einstein equations with effective sources in general form which are similar to Einstein-Yang-Mills-Higgs, EYMH, systems in higher-dimensions and with generalized gaugelike interactions. It is possible to consider associated $S U(3)$ structures and solve generalized BPS equations and Bianchi identities. A crucial difference from former approaches is that our geometric methods allow us to work with generating and integration functions for off-diagonal metrics and connections transforming equations of motion into nonlinear systems of partial differential equations, PDEs. In particular, we can reproduce former results for a diagonalizable ansatz with dependence on radial and warping coordinates as solutions of ordinary differential equations, ODEs.

To illustrate the power and importance of the AFDM as the most general geometric method of constructing analytic solutions of (modified) motion/gravitational and field equations, we show how this formalism can be applied for generating $\mathrm{N}$ adapted (i.e. adapted to nonlinear connection structures) YM and instanton configurations with possible associated $S U(3)$ nonholonomic structures; see the associated work [48]. New classes of exact solutions describing small parametric modifications of Kerr metrics with effective string sources are provided. We show that in a certain sense, a large class of physical effects in modified gravity models like $R^{2}$ can be equivalently modeled/explained by nonholonomic constraints and effective sources in heterotic string gravity. In explicit form, exact/parameteric extra dimension deformations of the black hole metrics in 6-d and 10-d gravity with NS-3 form and 6-d almost-Kähler internal spaces are constructed and analysed.

Finally, we emphasize that there are a plethora of future directions which can be pursued using our methods and results as starting points. This includes the construction of cosmological solutions in the heterotic string gravity and/or the study of smooth compact nonholonomic varieties in both heterotic and geometric flow context. Similar analyses can be performed in type II string theory in particular, including the Ramond-Ramond sector and/or considering geometric flows on internal spaces. We worked to the lowest order of $\alpha^{\prime}$ but possibilities in the AFDM exist to extend the constructions to higher orders.

Acknowledgements The SV research is for the QGR-Topanga with a former partial support by IDEI, PN-II-ID-PCE-2011-3-0256, and DAAD. He is grateful to N. Mavromatos, D. Lüst, O. Lechtenfeld, S. D. Odintsov and C. Castro Perelman for valuable discussions and support. This work contains also a summary of results of a talk at GR21 in NY.

Open Access This article is distributed under the terms of the Creative Commons Attribution 4.0 International License (http://creativecomm ons.org/licenses/by/4.0/), which permits unrestricted use, distribution, and reproduction in any medium, provided you give appropriate credit to the original author(s) and the source, provide a link to the Creative Commons license, and indicate if changes were made. Funded by $\mathrm{SCOAP}^{3}$.

\section{References}

1. A.S. Haupt, O. Lechtenfeld, E.T. Musaev, Order $\alpha^{\prime}$ heterotic domain walls with warped nearly Kähler geometry. JHEP 1411, 152 (2014)

2. D. Harland, C. Nölle, Instantons and Killing spinors. JHEP 1203, $082(2012)$

3. M. Graña, Flux compactifications in string theory: a comprehensive review. Phys. Rep. 423, 91-158 (2006)

4. B. Wecht, Lectures on nongeometric flux compactifications. Class. Quantum Gravity 24, S773-S794 (2007)

5. M.R. Douglas, S. Kachru, Flux compactification. Rev. Mod. Phys. 79, 733-796 (2009)

6. R. Blumenhagen, B. Körs, D. Lüst, S. Stieberger, Four-dimensional string compactifications with D-branes, orientifolds and fluxes. Phys. Rep. 445, 1-193 (2007)

7. H. Samtleben, Lectures on gauged supergravity and flux compactifications. Class. Quantum Gravity 25, 214002 (2008)

8. S. Vacaru, Covariant renormalizable anisotropic theories and offdiagonal Einstein-Yang-Mills-Higgs solutions. EPL 96, 50001 (2011) 
9. S. Vacaru, Two-connection renormalization and nonholonomic gauge models of Einstein gravity. Int. J. Geom. Methods Mod. Phys. 7, 713-744 (2010)

10. S. Vacaru, Einstein gravity as a nonholonomic almost Kähler geometry, Lagrange-Finsler variables, and deformation quantization. J. Geom. Phys. 60, 1289-1305 (2010)

11. S. Vacaru, Branes and quantization for an A-model complexification of Einstein gravity in almost Kähler variables. Int. J. Geom. Methods Mod. Phys. 6, 873-909 (2009)

12. S. Ferrara, A. Kehagias, A. Riotto, The imaginary Starobinsky model. Fortsch. Phys. 62, 573 (2014)

13. C. Kounnas, D. Lüst, N. Toumbas, $\mathcal{R}^{2}$ inflation from scale invariant supergravity and anomaly free superstrings with fluxes. Fortsch. Phys. 63, 12-35 (2015)

14. A. Kehagias, C. Kounnas, D. Lüst, A. Riotto, Black hole solutions in $R^{2}$ gravity. JHEP 5, 143 (2015)

15. S. Basilakos, P.C. Stavrinos, Cosmological equivalence between the Finsler-Randers spacetime in DGP gravity models. Phys. Rev. D 87, 043506 (2013)

16. S. Nojiri, S.D. Odintsov, V.K. Oikonomou, E.N. Saridakis, Singular cosmological evolution using canonical and ghost scalar fields. JCAP 1509, 044 (2015)

17. S. Basilakos, N.E. Mavromatos, J. Sola, Dynamically broken supergravity, Starobinsky-type inflation and running vacuum: towards a fundamental cosmic picture. arXiv: 1505.04434

18. S. Basilakos, A.P. Kouretsis, E.N. Saridakis, P. Stavrinos, Resembling dark energy and modified gravity with Finsler-Randers cosmology. Phys. Rev. D 88, 123510 (2013)

19. N.E. Mavromatos, V.A. Mitsou, S. Sarkar, A. Vergou, Implications of a stochastic microscopic Finsler cosmology. Eur. Phys. J. C 72, $1956(2012)$

20. S. Nojiri, S.D. Odintsov, Introduction to modified gravity and gravitational alternative for dark energy. Int. J. Geom. Methods Mod. Phys. 4, 115-146 (2007)

21. S. Capozziello, V. Faraoni, Beyond Einstein Gravity; A Survey of Gravitational Theories for Cosmology and Astrophysics, Fundamental Theories of Physics, vol. 170 (Springer, Netherlands, 2011), p. 467

22. G. Gabadadze, General relativity with an auxiliary dimension. Phys. Lett. B 681, 89 (2009)

23. S. Hassan, R.A. Rosen, Resolving the ghost problem in non-linear massive gravity. Phys. Rev. Lett. 108, 041101 (2012)

24. S. Vacaru, Modified dispersion relations in Horava-Lifshitz gravity and Finsler brane models. Gen. Relat. Gravit. 44, 1015-1042 (2012)

25. S. Vacaru, D. Singleton, Singleton, warped, anisotropic wormhole/soliton configurations in vacuum 5D gravity. Class. Quantum Gravity 19, 2793-2811 (2002)

26. P. Stavrinos, S. Vacaru, Cyclic and ekpyrotic universes in modified Finsler osculating gravity on tangent Lorentz bundles. Class. Quantum Gravity 30, 055012 (2013)

27. S. Vacaru, Ghost-free massive $f(R)$ theories modelled as effective Einstein spaces \& cosmic acceleration. Eur. Phys. J. C 74, 3132 (2014)

28. S. Vacaru, Equivalent off-diagonal cosmological models and ekpyrotic scenarios in $\mathrm{f}(\mathrm{R})$-modified massive and Einstein gravity. Eur. Phys. J. C 75, 176 (2015)

29. E. Elizalde, S. Vacaru, Effective Einstein cosmological spaces for non-minimal modified gravity. Gen. Relat. Gravit. 47, 64 (2015)

30. S. Vacaru, Off-diagonal ekpyrotic scenarios and equivalence of modified, massive and/or Einstein gravity. Phys. Lett. B 752, 2733 (2016)

31. D. Kramer, H. Stephani, E. Herdlt, M.A.H. MacCallum, Exact Solutions of Einstein's Field Equations, 2nd edn. (Cambridge University Press, Cambridge, 2003)
32. J.B. Griffiths, J. Podolský, Exact Space-Times in Einstein's General Relativity. Cambridge Monographs on Mathematical Physics (Cambridge University Press, Cambridge, 2009)

33. R. Geroch, A method for generating solutions of Einstein's equations. J. Math. Phys. 12, 918-925 (1971)

34. R. Geroch, A method for generating new solutions of Einstein's equations. II. J. Math Phys. 13, 394-404 (1972)

35. S. Vacaru, Parametric nonholonomic frame transforms and exact solutions in gravity. Int. J. Geom. Methods Mod. Phys. 4, 12851334 (2007)

36. S. Vacaru, Anholonomic soliton-dilaton and black hole solutions in general relativity. JHEP 04, 009 (2001)

37. S. Vacaru, Locally anisotropic kinetic processes and thermodynamics in curved spaces. Ann. Phys. (N.Y.) 290, 83-123 (2001)

38. S. Vacaru, Exact solutions with noncommutative symmetries in Einstein and gauge gravity. J. Math. Phys. 46, 042503 (2005)

39. S. Vacaru, On general solutions in Einstein gravity. Int. J. Geom. Methods Mod. Phys. 8, 9-21 (2011)

40. S. Vacaru, On general solutions in Einstein and high dimensional gravity. Int. J. Theor. Phys. 49, 884-913 (2010)

41. S. Vacaru, Decoupling of field equations in Einstein and modified gravity. J. Phys. Conf. Ser. 543, 012021 (2013)

42. S. Vacaru, E. Veliev, E. Yazici, A geometric method of constructing exact solutions in modified $\mathrm{f}(\mathrm{R}, \mathrm{T})$ gravity with Yang-Mills and Higgs Interactions. IJGMMP 11, 1450088 (2014)

43. T. Gheorghiu, O. Vacaru, S. Vacaru, Off-diagonal deformations of Kerr black holes in Einstein and modified massive gravity and higher dimensions. EPJC 74, 3152 (2014)

44. T. Gheorghiu, O. Vacaru, S. Vacaru, Modified dynamical supergravity breaking and off-diagonal super-Higgs effects. Class. Quantum Gravity 32, 065004 (2015)

45. M.B. Green, J.H. Schwarz, Anomaly cancellation in supersymmetric $D=10$ gauge theory and superstring theory. Phys. Lett. B 149, 117-122 (1984)

46. D.J. Gross, J.A. Harvey, E.J. Martinec, R. Rohm, Heterotic string theory. 1. The free heterotic string. Nucl. Phys. B 256, 253-284 (1985)

47. D.J. Gross, J.A. Harvey, E.J. Martinec, R. Rohm, Heterotic string theory. 2. The interacting heterotic string. Nucl. Phys. B 267, 75124 (1986)

48. L. Bubuianu, K. Irwin, S. Vacaru, Heterotic supergravity with internal almost-Kähler configurations and Gauge $S O(32)$, or $E_{8} \times E_{8}$, instantons. arXiv:1611.00223

49. T. Nieuwenhuizen, Exact Schwarzschild-de Sitter black holes in a family of massive gravity models. Phys. Rev. D 84, 024038 (2011)

50. S. Koyama, G. Niz, G. Tasinato, The self-accelerating universe with vectors in massive gravity. JHEP 1112, 065 (2011)

51. S. Vacaru, Finsler branes and quantum gravity phenomenology with Lorentz symmetry violations. Class. Quantum Gravity 28, 215991 (2011)

52. C.W. Misner, K.S. Thorne, J.A. Wheeler, Gravitation (Freeman, 1973)

53. S. Vacaru, Superstrings in higher order extensions of Finsler superspaces. Nucl. Phys. B 434, 590-656 (1997)

54. S. Vacaru, Locally anisotropic gravity and strings. Ann. Phys. (NY) 256, 39-61 (1997)

55. S. Vacaru, F.C. Popa, Dirac spinor waves and solitons in anisotropic Taub-NUT spaces. Class. Quantum Gravity 18, 4921-4938 (2001)

56. S. Vacaru, O. Tintareanu-Mircea, Anholonomic frames, generalized Killing equations, and anisotropic Taub NUT spinning spaces. Nucl. Phys. B 626, 239-264 (2002)

57. S. Vacaru, Spectral functionals, nonholonomic Dirac operators, and noncommutative Ricci flows. J. Math. Phys. 50, 073503 (2009)

58. C. Hull, Anomalies, ambiguities and superstrings. Phys. Lett. B 167, 51-55 (1986) 
59. S. Ivanov, Heterotic supersymmetry, anomaly cancellation and equations of motion. Phys. Lett 685, 190-196 (2010)

60. E. Bergshoeff, M. de Roo, The quardic effective action of the heterotic string and supersymmetry. Nucl. Phys. B 328, 439-468 (1989)

61. K. Becker, S. Sethi, Torsional heterotic geometries. Nucl. Phys. B 820, 1-31 (2009)

62. S. Vacaru, The algebraic index theorem and Fedosov quantization of Lagrange-Finsler and Einstein spaces. J. Math. Phys. 54, 073511 (2013)

63. S. Vacaru, Almost Kähler Ricci flows and Einstein and LagrangeFinsler structures on Lie algebroids. Medit. J. Math. 12, 1397-1427 (2015)

64. G.T. Horowitz, A. Strominger, Black strings and p-branes. Nucl. Phys. B 360, 197-209 (1991)

65. K.-P. Gemmer, A.S. Haupt, O. Lechtenfeld, C. Nölle, A.D. Popov, Heterotic sting plus five-brane systems with asymptotic $A d S_{3}$. Adv. Theor. Math. Phys. 17, 771-827 (2013)
66. M. Klaupt, A. Lukas, C. Matti, E.E. Svanes, Moduli stabilising in heterotic nearly Kähler compactifications. JHEP 1301, 015 (2013)

67. J. Gray, M. Larfors, D. Lüst, Heterotic domain wall solutions and SU (3) structure manifolds. JHEP 1208, 099 (2012)

68. A. Lukas, C. Matti, G-structures and domain walls in heterotic theories. JHEP 1101, 151 (2011)

69. S. Chiossi, S. Salamon, The intrinsic torsion of SU(3) and G_2 structures. in Differential Geometry, Valencia 2001 (World Scientific Publishing, Singapore, 2002), pp. 115-133. arXiv:math/0202282

70. T. Gheorghiu, V. Ruchin, O. Vacaru, S. Vacaru, Geometric flows and Perelman's thermodynamics for black ellipsoids in $R^{2}$ and Einstein gravity theories. Ann. Phys. N.Y. 369, 1 (2016)

71. M. Heusler, Black Hole Uniqueness Theorems (Cambridge University Press, Cambridge, 1996)

72. S. Vacaru, Hidden symmetries for ellipsoid-solitonic deformations of Kerr-Sen black holes and quantum anomalies. Eur. Phys. J. C 73, 2287 (2013) 